\title{
THE FORMATION AND EVOLUTION OF PLANETARY SYSTEMS: DESCRIPTION OF THE SPITZER LEGACY SCIENCE DATABASE
}

\author{
John M. Carpenter, ${ }^{1}$ Jeroen Boumman, ${ }^{2}$ Murray D. Silverstone, ${ }^{3}$ Jinyoung Serena Kim, ${ }^{4}$ John Stauffer, ${ }^{5}$ \\ Martin Cohen, ${ }^{6}$ Dean C. Hines, ${ }^{7}$ Michael R. Meyer, ${ }^{4}$ and Nathan Crockett ${ }^{8}$ \\ Received 2008 March 31; accepted 2008 July 26
}

\begin{abstract}
We present the science database produced by the Formation and Evolution of Planetary Systems (FEPS) Spitzer Legacy program. Data reduction and validation procedures for the IRAC, MIPS, and IRS instruments are described in detail. We also derive stellar properties for the FEPS sample from available broadband photometry and spectral types, and present an algorithm to normalize Kurucz synthetic spectra to optical and near-infrared photometry. The final FEPS data products include IRAC and MIPS photometry for each star in the FEPS sample and calibrated IRS spectra.
\end{abstract}

Subject headings: circumstellar matter — infrared: stars — planetary systems: formation

Online material: tar file

\section{INTRODUCTION}

The Formation and Evolution of Planetary Systems (FEPS) Spitzer Legacy program (Meyer et al. 2006) was designed to characterize the evolution of circumstellar gas and dust around solartype stars between ages of $3 \mathrm{Myr}$ and $3 \mathrm{Gyr}$. To achieve these goals, FEPS obtained spectrophotometric observations with the Spitzer Space Telescope (Werner et al. 2004) for a sample of 328 stars (see Meyer et al. 2006 for a description of the sample). The observing strategy was to measure the spectral energy distribution (SED) between wavelengths of 3.6 and $70 \mu \mathrm{m}$ with IRAC (Infrared Array Camera; Fazio et al. 2004) and MIPS (Multiband Imaging Photometer for Spitzer; Rieke et al. 2004) photometry, and between 8 and $35 \mu \mathrm{m}$ with low-resolution IRS (Infrared Spectrograph; Houck et al. 2004) spectra. In addition, the FEPS program obtained MIPS $160 \mu \mathrm{m}$ photometry for 80 stars to search for colder dust, and high-resolution IRS spectra for 33 sources to probe for circumstellar gas.

The FEPS team has produced several studies on the incidence of dusty debris disks around solar type stars, including the discovery of a debris system in the initial Spitzer observations (Meyer et al. 2004), a census of warm debris (Stauffer et al. 2005; Silverstone et al. 2006; Hines et al. 2006; Meyer et al. 2008), the identification of Kuiper Belt analogs (Kim et al. 2005; Hillenbrand et al. 2008), and an investigation of debris disks around stars with known planets (Moro-Martín et al. 2007). The FEPS team has also analyzed the processing of dust in optically thick, primordial disks (Bouwman et al. 2008), and has produced a series of papers on the evolution of gas in solar-type stars (Hollenbach et al. 2005; Pascucci et al. 2006, 2007).

This paper describes the data reduction procedures for IRAC $(3.6,4.5$, and $8 \mu \mathrm{m})$ and MIPS (24 and $70 \mu \mathrm{m})$ images and IRS

\footnotetext{
1 Department of Astronomy, California Institute of Technology, Mail Code 105-24, 1200 East California Boulevard, Pasadena, CA 91125.

2 Max-Planck-Institut für Astronomie, D-69117 Heidelberg, Germany.

3 Eureka Scientific Inc., 113 Castlefern Drive, Cary NC 25713.

4 Steward Observatory, The University of Arizona, 933 North Cherry Avenue, Tucson, AZ 85721.

5 Spitzer Science Center, California Institute of Technology, Mail Code 314-6, 1200 East California Boulevard, Pasadena, CA 91125.

6 Radio Astronomy Laboratory, University of California, Berkeley, CA 94720.

7 Space Science Institute, 4750 Walnut Street, Suite 205, Boulder, CO 80301.

8 Department of Astronomy, University of Michigan, Ann Arbor, MI 48109.
}

low-resolution spectra obtained by the FEPS program. Data reduction methods for the MIPS $160 \mu \mathrm{m}$ images and IRS highresolution spectra are discussed in Kim et al. (in preparation) and Pascucci et al. (2006), respectively. The adopted reduction procedures for the IRAC, MIPS $24 \mu \mathrm{m}$, MIPS $70 \mu \mathrm{m}$, and IRS observations are presented in $\S \S 2-5$. We also investigate the effects of source confusion on the 24 and $70 \mu$ m photometry $(\S 6)$ and the relative calibration accuracy between Spitzer instruments $(\S 7)$. The series of FEPS papers frequently utilized synthetic spectra derived from Kurucz model atmospheres to infer the presence of infrared excesses diagnostic of circumstellar dust. In the Appendices, we describe the data and algorithm used to obtain normalized synthetic spectra for individual stars. The primary data products from the FEPS program are a tabulation of IRAC and MIPS photometry presented in Table 1, and extracted, calibrated spectra which are available electronically.

\section{IRAC}

IRAC produces images in four channels at wavelengths of 3.6, 4.5, 5.8, and $8.0 \mu \mathrm{m}$ with bandwidths of $0.75,1.01,1.42$, and $2.93 \mu \mathrm{m}$, respectively (Fazio et al. 2004). The FEPS team obtained IRAC observations for 311 of the 328 stars in the sample. The remaining 17 objects were observed by other Spitzer programs, including 16 Hyades stars in a Guaranteed Time Observations (GTO) program led by G. Fazio, and one source (ScoPMS 214) in the Upper Sco OB Association by the c2d Legacy Program (Evans et al. 2003).

FEPS IRAC observations were conducted in subarray mode with a four-point dither pattern and the medium dither scale. The locations of the four dither positions on the array are the same for each source to within the pointing accuracy of the spacecraft ( $1 \sigma<1^{\prime \prime}$ radial; Werner et al. 2004). In subarray mode, each IRAC band is observed separately where a $32 \times 32$ pixel section $\left(39^{\prime \prime} \times 39^{\prime \prime}\right)$ in a corner of the $256 \times 256$ pixel full-array $\left(5.2^{\prime} \times\right.$ $5.2^{\prime}$ ) is read out at frame times of $0.02,0.10$, or $0.40 \mathrm{~s}$. At each dither position, 64 images are taken at the same frame time for a total of 256 images per band, with the same frame time for each band and a given source. The total on-source integration time per band is then 5.12, 25.6, and 102.4 $\mathrm{s}$ for frame times of $0.02,0.10$, and $0.40 \mathrm{~s}$, respectively. The frame time was selected on a sourceby-source basis to achieve a high signal-to-noise ratio $(\mathrm{S} / \mathrm{N})$ on the stellar photosphere without saturating the detector. Five FEPS 
TABLE 1

IRAC AND MIPS PHOTOMETRY

\begin{tabular}{|c|c|c|c|c|c|c|c|c|c|c|c|c|c|c|c|c|}
\hline \multirow[b]{4}{*}{ SOURCE } & \multicolumn{6}{|c|}{ IRAC } & \multicolumn{4}{|c|}{ MIPS } & \multirow{4}{*}{$\begin{array}{l}\text { IRAC FraME } \\
\text { Time } \\
\text { (s) }\end{array}$} & \multirow{4}{*}{$\begin{array}{c}\text { MIPS } 24 \mu \mathrm{m} \\
\text { DCE TIME } \\
\text { (s) }\end{array}$} & \multirow{3}{*}{\multicolumn{2}{|c|}{ AOR KeY }} & \multirow{3}{*}{\multicolumn{2}{|c|}{ FLAGS $^{\mathrm{a}}$}} \\
\hline & \multicolumn{2}{|c|}{$3.6 \mu \mathrm{m}$} & \multicolumn{2}{|c|}{$4.5 \mu \mathrm{m}$} & \multicolumn{2}{|c|}{$8 \mu \mathrm{m}$} & \multicolumn{2}{|c|}{$24 \mu \mathrm{m}$} & \multicolumn{2}{|c|}{$70 \mu \mathrm{m}$} & & & & & & \\
\hline & \multirow{2}{*}{$\begin{array}{c}S_{\nu} \\
\text { (mJy) }\end{array}$} & \multirow{2}{*}{$\begin{array}{l}\sigma_{\mathrm{int}} \\
(\mathrm{mJy})\end{array}$} & \multirow{2}{*}{$\begin{array}{c}S_{\nu} \\
(\mathrm{mJy})\end{array}$} & \multirow{2}{*}{$\begin{array}{l}\sigma_{\text {int }} \\
(\mathrm{mJy})\end{array}$} & \multirow{2}{*}{$\begin{array}{l}S_{\nu} \\
\text { (mJy) }\end{array}$} & & & & & & & & & & & \\
\hline & & & & & & (mJy) & (mJy) & (mJy) & (mJy) & (mJy) & & & IRAC & MIPS & IRAC & MIPS \\
\hline 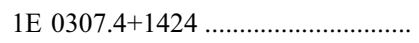 & 91.21 & 0.66 & 57.60 & 0.70 & 20.51 & 0.14 & 2.60 & 0.03 & -10.8 & 5.4 & 0.40 & 9.96 & 5296896 & 5297408 & & \\
\hline 1E $0324.1-2012 \ldots \ldots \ldots \ldots \ldots \ldots$ & 86.59 & 0.62 & 55.47 & 0.68 & 19.53 & 0.13 & 2.50 & 0.02 & -1.7 & 3.7 & 0.40 & 9.96 & 5297664 & 5298176 & & \\
\hline 1RXS J025216.9+361658 …........... & 321.18 & 2.31 & 202.24 & 2.47 & 77.25 & 0.51 & 8.81 & 0.08 & -0.6 & 4.3 & 0.10 & 9.96 & 5256960 & 5257472 & & 2 \\
\hline 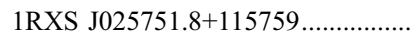 & 121.01 & 0.87 & 76.26 & 0.93 & 27.93 & 0.18 & 3.27 & 0.04 & 1.3 & 5.4 & 0.10 & 9.96 & 5261568 & 5262080 & & \\
\hline 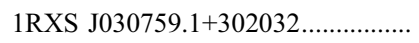 & 313.33 & 2.26 & 196.62 & 2.40 & 71.77 & 0.47 & 8.13 & 0.08 & 2.0 & 5.0 & 0.10 & 2.62 & 5313536 & 5314048 & & \\
\hline 1RXS J031644.0+192259 ………..... & 55.94 & 0.40 & 35.50 & 0.43 & 12.87 & 0.09 & 1.57 & 0.03 & -9.4 & 5.9 & 0.40 & 9.96 & 5302272 & 5302784 & & \\
\hline 1RXS J031907.4+393418.................... & 47.01 & 0.34 & 29.19 & 0.36 & 10.88 & 0.07 & 1.31 & 0.02 & -3.0 & 4.3 & 0.40 & 9.96 & 5257728 & 5258240 & & \\
\hline 1RXS J034423.3+281224.................... & 389.93 & 2.81 & 246.84 & 3.01 & 88.38 & 0.58 & 10.01 & 0.09 & -0.4 & 5.5 & 0.10 & 2.62 & 5258496 & 5259008 & & \\
\hline 1RXS J035028.0+163121 ……........ & 107.74 & 0.78 & 68.39 & 0.83 & 24.39 & 0.16 & 2.95 & 0.03 & -10.1 & 6.6 & 0.10 & 9.96 & 5263104 & 5263616 & & \\
\hline 1RXS J043243.2-152003 ……........ & 106.89 & 0.77 & 66.74 & 0.81 & 23.97 & 0.16 & 2.92 & 0.03 & -2.4 & 3.5 & 0.10 & 9.96 & 5244416 & 5244928 & & \\
\hline 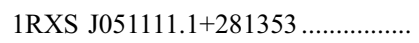 & 223.49 & 1.61 & 139.47 & 1.70 & 53.61 & 0.35 & 7.77 & 0.07 & 1.4 & 11.0 & 0.10 & 2.62 & 5246720 & 5247232 & & \\
\hline 1RXS J053650.0+133756 ….............. & 179.78 & 1.29 & 112.07 & 1.37 & 41.56 & 0.27 & 4.93 & 0.04 & -0.2 & 11.0 & 0.10 & 9.96 & 5247488 & 5248000 & & \\
\hline 2RE J0255+474 & 396.39 & 2.85 & 248.21 & 3.03 & 90.62 & 0.60 & 10.30 & 0.09 & -1.3 & 4.6 & 0.10 & 2.62 & 5349632 & 5350144 & 1 & \\
\hline 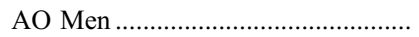 & 568.24 & 4.09 & 359.74 & 4.81 & 130.52 & 0.86 & 16.16 & 0.15 & 4.9 & 3.4 & 0.02 & 2.62 & 5222144 & 5222656 & & \\
\hline AP 93 & 56.05 & 0.40 & 34.91 & 0.43 & 12.96 & 0.09 & 1.62 & 0.02 & 5.3 & 10.2 & 0.40 & 9.96 & 5282304 & 5282816 & & \\
\hline B102 & 88.83 & 0.68 & 56.44 & 0.77 & 20.52 & 0.14 & 2.54 & 0.11 & -15.0 & 22.1 & 0.40 & 9.96 & 5275392 & 5275904 & & \\
\hline 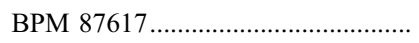 & 234.87 & 1.69 & 148.05 & 1.81 & 54.85 & 0.36 & 6.59 & 0.06 & -3.0 & 4.3 & 0.10 & 2.62 & 5348864 & 5349376 & & \\
\hline HD 105 & 1022.72 & 7.36 & 645.37 & 7.87 & 230.66 & 1.52 & 28.29 & 0.25 & 141.2 & 10.4 & 0.02 & 2.62 & 5295360 & 5295872 & & \\
\hline 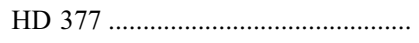 & 1029.07 & 7.41 & 648.56 & 7.91 & 234.70 & 1.67 & 36.58 & 0.33 & 162.0 & 12.6 & 0.02 & 2.62 & 5268480 & 5268992 & & \\
\hline HD 691 & 967.52 & 6.97 & 597.48 & 7.29 & 218.32 & 1.44 & 25.01 & 0.23 & 8.3 & 7.2 & 0.02 & 2.62 & 5345024 & 5345536 & & \\
\hline HD 984 & 1050.29 & 7.56 & 662.15 & 8.08 & 236.77 & 1.56 & 26.88 & 0.24 & -9.4 & 8.4 & 0.02 & 2.62 & 5271552 & 5272064 & & 2 \\
\hline HD 6434 & 952.27 & 6.86 & 603.33 & 7.36 & 215.10 & 1.42 & 23.95 & 0.22 & 8.0 & 7.4 & 0.02 & 2.62 & 5439232 & 5439744 & & \\
\hline HD 6963 & 1211.31 & 8.72 & 752.85 & 9.19 & 271.46 & 1.79 & 32.53 & 0.29 & 44.0 & 8.0 & 0.02 & 2.62 & 5395712 & 5396224 & & \\
\hline HD 7661 & 1428.00 & 10.28 & 887.28 & 10.82 & 322.51 & 2.13 & 36.03 & 0.32 & 4.4 & 7.9 & 0.02 & 2.62 & 5370624 & 5371136 & & \\
\hline 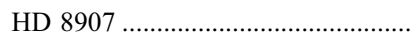 & 1918.20 & 13.81 & 1223.73 & 14.93 & 427.28 & 2.82 & 51.28 & 0.46 & 247.4 & 9.3 & 0.02 & 2.62 & 5361920 & 5362432 & & 2 \\
\hline HD 8941 & 2006.73 & 14.45 & 1266.43 & 15.45 & 448.80 & 2.96 & 49.89 & 0.45 & 4.5 & 9.3 & 0.02 & 2.62 & 5413888 & 5414400 & & \\
\hline HD 9472 & 1088.63 & 7.84 & 678.21 & 8.27 & 242.00 & 1.60 & 27.28 & 0.25 & 1.1 & 9.5 & 0.02 & 2.62 & 5391872 & 5392384 & & \\
\hline HD 11850............................................. & 952.41 & 6.86 & 595.66 & 7.27 & 211.74 & 1.78 & 24.05 & 0.22 & -4.0 & 9.6 & 0.02 & 2.62 & 5375232 & 5375744 & & \\
\hline HD 12039 ........................................ & 747.34 & 5.38 & 470.88 & 5.75 & 170.38 & 1.13 & 25.65 & 0.23 & 3.5 & 8.1 & 0.02 & 2.62 & 5310464 & 5310976 & & \\
\hline HD 13382 & 1391.67 & 10.02 & 881.73 & 10.76 & 313.35 & 2.07 & 36.27 & 0.33 & 3.2 & 9.5 & 0.02 & 2.62 & 5372928 & 5373440 & & \\
\hline HD 13507 ...................................... & 1715.75 & 12.35 & 1071.21 & 13.07 & 389.82 & 2.57 & 42.99 & 0.39 & 4.4 & 7.4 & 0.02 & 2.62 & 5390336 & 5390848 & & \\
\hline HD 13531 & 1552.04 & 11.18 & 964.04 & 11.76 & 349.37 & 2.31 & 39.14 & 0.35 & 1.8 & 8.0 & 0.02 & 2.62 & 5372160 & 5372672 & & \\
\hline HD 13974 & 14705.79 & 105.88 & 9279.19 & 113.21 & 3299.77 & 21.78 & 374.1 & 3.4 & 46.0 & 9.0 & 0.02 & 2.62 & 5410816 & 5411328 & & \\
\hline HD 15526 & 182.75 & 1.32 & 113.77 & 1.39 & 40.84 & 0.27 & 4.89 & 0.04 & -4.5 & 3.9 & 0.10 & 9.96 & 5263872 & 5264384 & & \\
\hline HD 17925 & 7280.60 & 52.42 & 4520.57 & 55.15 & 1644.56 & 10.85 & 193.6 & 1.7 & 57.0 & 11.6 & 0.02 & 2.62 & 5306112 & 4036352 & & 4 \\
\hline 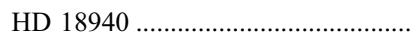 & 1815.57 & 13.07 & 1130.20 & 13.79 & 405.23 & 2.67 & 45.58 & 0.41 & -4.7 & 9.9 & 0.02 & 2.62 & 5388032 & 5388544 & & \\
\hline 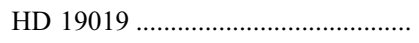 & 1664.30 & 11.98 & 1050.85 & 12.82 & 371.56 & 2.45 & 42.21 & 0.38 & 3.9 & 10.3 & 0.02 & 2.62 & 5407232 & 5407744 & & \\
\hline HD 19668 & 605.66 & 5.12 & 382.24 & 4.66 & 134.35 & 1.01 & 18.74 & 0.17 & -2.0 & 9.2 & 0.02 & 2.62 & 5340416 & 5340928 & & \\
\hline 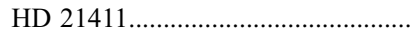 & 1008.04 & 7.26 & 627.22 & 7.65 & 225.56 & 1.49 & 25.14 & 0.23 & 3.8 & 7.6 & 0.02 & 2.62 & 5389568 & 5390080 & & \\
\hline HD 22179 & 311.69 & 2.24 & 196.20 & 2.39 & 71.03 & 0.47 & 11.10 & 0.10 & 35.9 & 10.3 & 0.10 & 2.62 & 5262336 & 5262848 & & \\
\hline HD 25300 & 649.30 & 4.67 & 398.75 & 4.87 & 152.33 & 1.41 & 18.61 & 0.17 & 0.7 & 4.8 & 0.02 & 2.62 & 5350400 & 5350912 & & \\
\hline HD 25457 . & 6259.72 & 45.07 & 3956.34 & 48.27 & 1412.34 & 9.32 & 205.8 & 1.9 & 307.2 & 9.2 & 0.02 & 2.62 & 5308160 & 5308672 & & \\
\hline HD 26182 & 227.33 & 1.64 & 144.51 & 1.76 & 52.11 & 0.34 & 5.94 & 0.07 & 1.6 & 8.8 & 0.10 & 2.62 & 5358848 & 5359360 & 1 & \\
\hline
\end{tabular}


TABLE 1-Continued

\begin{tabular}{|c|c|c|c|c|c|c|c|c|c|c|c|c|c|c|c|c|}
\hline \multirow[b]{4}{*}{ Source } & \multicolumn{6}{|c|}{ IRAC } & \multicolumn{4}{|c|}{ MIPS } & \multirow{4}{*}{$\begin{array}{c}\text { IRAC Frame } \\
\text { Time } \\
\text { (s) }\end{array}$} & \multirow{4}{*}{$\begin{array}{c}\text { MIPS } 24 \mu \mathrm{m} \\
\text { DCE TIME } \\
\text { (s) }\end{array}$} & \multirow{3}{*}{\multicolumn{2}{|c|}{ AOR KeY }} & \multirow{3}{*}{\multicolumn{2}{|c|}{ FlaGs $^{\mathrm{a}}$}} \\
\hline & \multicolumn{2}{|c|}{$3.6 \mu \mathrm{m}$} & \multicolumn{2}{|c|}{$4.5 \mu \mathrm{m}$} & \multicolumn{2}{|c|}{$8 \mu \mathrm{m}$} & \multicolumn{2}{|c|}{$24 \mu \mathrm{m}$} & \multicolumn{2}{|c|}{$70 \mu \mathrm{m}$} & & & & & & \\
\hline & & & & & & & & & 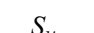 & & & & & & & \\
\hline & (mJy) & $(\mathrm{mJy})$ & (mJy) & (mJy) & (mJy) & (mJy) & (mJy) & (mJy) & (mJy) & (mJy) & & & IRAC & MIPS & IRAC & MIPS \\
\hline HD $26990 \ldots \ldots \ldots \ldots \ldots \ldots$ & 1286.97 & 9.27 & 804.17 & 9.81 & 289.30 & 1.91 & 32.75 & 0.29 & 9.4 & 9.0 & 0.02 & 2.62 & 5391104 & 5391616 & & \\
\hline HD $27466 \ldots \ldots \ldots \ldots \ldots$ & 883.45 & 6.36 & 557.40 & 6.80 & 198.51 & 1.31 & 21.90 & 0.20 & 1.7 & 7.6 & 0.02 & 2.62 & 5411584 & 5412096 & & \\
\hline HD $28495 \ldots \ldots \ldots \ldots \ldots \ldots$ & 1463.51 & 10.54 & 911.59 & 11.12 & 331.19 & 2.19 & 38.13 & 0.34 & -7.0 & 10.3 & 0.02 & 2.62 & 5366016 & 5366528 & & \\
\hline 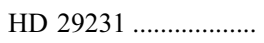 & 1332.22 & 9.59 & 821.42 & 10.02 & 297.62 & 1.96 & 33.14 & 0.30 & 3.8 & 7.2 & 0.02 & 2.62 & 5405696 & 5406208 & & \\
\hline HD $31143 \ldots \ldots \ldots \ldots$ & 900.01 & 6.48 & 556.10 & 6.78 & 203.33 & 1.34 & 22.69 & 0.20 & 2.9 & 7.8 & 0.02 & 2.62 & 5401088 & 5401600 & & \\
\hline 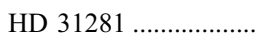 & 271.15 & 1.95 & 171.58 & 2.09 & 62.45 & 0.41 & 7.43 & 0.09 & 9.6 & 13.4 & 0.10 & 2.62 & 5254912 & 5255424 & & \\
\hline HD $31392 \ldots \ldots \ldots \ldots \ldots \ldots$ & 1431.93 & 10.31 & 891.69 & 10.88 & 321.84 & 2.12 & 36.88 & 0.33 & 81.6 & 8.4 & 0.02 & 2.62 & 5398016 & 5398528 & & \\
\hline 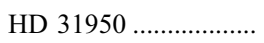 & 130.03 & 0.94 & 83.02 & 1.01 & 29.00 & 0.24 & 3.69 & 0.04 & -2.7 & 8.9 & 0.10 & 9.96 & 5303808 & 5304320 & 1 & \\
\hline 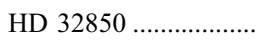 & 1487.55 & 10.71 & 929.44 & 11.34 & 336.42 & 2.22 & 38.37 & 0.35 & 2.2 & 11.9 & 0.02 & 2.62 & 5402624 & 5403136 & & \\
\hline HD 35850 & 3030.34 & 21.82 & 1917.88 & 23.40 & 690.67 & 4.56 & 83.52 & 0.75 & 40.3 & 7.5 & 0.02 & 2.62 & 5446912 & 5447424 & & \\
\hline 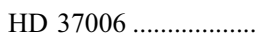 & 740.10 & 5.33 & 465.45 & 5.68 & 164.16 & 1.08 & 18.64 & 0.17 & -2.1 & 6.2 & 0.02 & 2.62 & 5388800 & 5389312 & & \\
\hline HD $37216 \ldots \ldots \ldots \ldots \ldots$ & 1122.32 & 8.08 & 694.71 & 8.47 & 250.81 & 1.66 & 28.03 & 0.25 & 6.7 & 9.3 & 0.02 & 2.62 & 5386496 & 5387008 & & \\
\hline 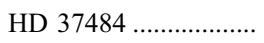 & 893.68 & 6.43 & 568.11 & 7.50 & 202.23 & 1.59 & 54.59 & 0.49 & 114.4 & 7.8 & 0.02 & 2.62 & 5306624 & 5307136 & & \\
\hline HD $37572 \ldots$ & 1362.34 & 9.81 & 848.38 & 10.35 & 310.18 & 2.05 & 35.26 & 0.32 & 5.7 & 7.6 & 0.02 & 2.62 & 6601472 & 6599680 & & \\
\hline HD $37962 \ldots \ldots \ldots \ldots$ & 883.69 & 6.36 & 553.46 & 6.75 & 198.17 & 1.45 & 22.62 & 0.20 & 16.5 & 7.4 & 0.02 & 2.62 & 5412352 & 5412864 & & \\
\hline 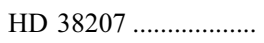 & 286.99 & 2.07 & 181.35 & 2.21 & 64.62 & 0.43 & 16.46 & 0.15 & 184.6 & 4.7 & 0.10 & 2.62 & 5363200 & 5363712 & & \\
\hline HD $38529 \ldots \ldots \ldots \ldots \ldots$ & 5893.09 & 42.43 & 3634.04 & 44.34 & 1339.97 & 8.84 & 149.6 & 1.3 & 75.3 & 11.2 & 0.02 & 2.62 & 5436928 & 5437440 & & \\
\hline 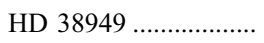 & 775.76 & 5.58 & 488.07 & 5.95 & 172.26 & 1.16 & 20.02 & 0.18 & 7.2 & 8.0 & 0.02 & 2.62 & 5339648 & 5340160 & & \\
\hline HD 40647 ............................ & 872.61 & 6.28 & 543.42 & 6.63 & 195.98 & 1.29 & 22.11 & 0.20 & 7.0 & 6.7 & 0.02 & 2.62 & 5384192 & 5384704 & & \\
\hline 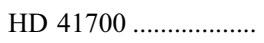 & 2693.24 & 19.39 & 1696.38 & 20.70 & 613.28 & 4.05 & 71.78 & 0.65 & 22.2 & 7.6 & 0.02 & 2.62 & 5365248 & 5365760 & & \\
\hline HD 43989 .................. & 719.52 & 5.18 & 454.19 & 5.54 & 163.22 & 1.21 & 21.14 & 0.19 & 7.1 & 9.8 & 0.02 & 2.62 & 6600704 & 6598912 & & \\
\hline 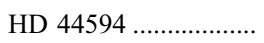 & 2593.89 & 18.68 & 1606.66 & 19.60 & 587.33 & 3.88 & 63.92 & 0.58 & 5.3 & 6.7 & 0.02 & 2.62 & 5444608 & 5445120 & & \\
\hline HD 45270 & 2675.06 & 19.26 & 1698.63 & 20.72 & 599.85 & 3.96 & 70.18 & 0.63 & 8.5 & 6.0 & 0.02 & 2.62 & 6601216 & 6599424 & & 2 \\
\hline HD 47875 & 364.71 & 2.95 & 230.96 & 3.70 & 81.40 & 2.08 & 9.70 & 0.09 & 0.0 & 3.7 & 0.02 & 2.62 & 5293824 & 5294336 & 1 & \\
\hline 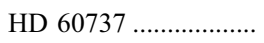 & 910.07 & 6.55 & 569.23 & 6.95 & 202.08 & 1.51 & 24.10 & 0.22 & 17.7 & 11.1 & 0.02 & 2.62 & 5267712 & 5268224 & & \\
\hline 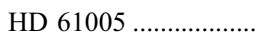 & 753.53 & 5.42 & 472.32 & 5.76 & 169.19 & 1.12 & 41.49 & 0.37 & 628.7 & 11.1 & 0.02 & 2.62 & 5266944 & 5267456 & & \\
\hline 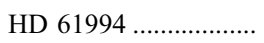 & 2103.13 & 15.14 & 1291.84 & 17.11 & 471.04 & 3.11 & 51.98 & 0.47 & 6.9 & 6.9 & 0.02 & 2.62 & 5394176 & 5394688 & & \\
\hline 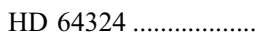 & 929.20 & 6.69 & 585.16 & 7.14 & 207.22 & 1.37 & 23.76 & 0.21 & 6.3 & 9.9 & 0.02 & 2.62 & 5400320 & 5400832 & & \\
\hline 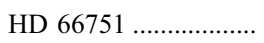 & 2683.46 & 19.32 & 1682.98 & 20.53 & 600.21 & 3.96 & 66.35 & 0.60 & 6.0 & 7.2 & 0.02 & 2.62 & 5408768 & 5409280 & & \\
\hline HD $69076 \ldots \ldots \ldots \ldots \ldots$ & 791.83 & 5.70 & 492.67 & 6.01 & 177.87 & 2.21 & 20.14 & 0.18 & 0.4 & 8.4 & 0.02 & 2.62 & 5421568 & 5422080 & & 2 \\
\hline 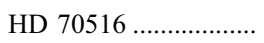 & 995.64 & 7.17 & 623.34 & 7.61 & 227.16 & 1.50 & 25.38 & 0.23 & 8.4 & 6.9 & 0.02 & 2.62 & 5292288 & 5292800 & & \\
\hline HD $70573 \ldots$ & 381.64 & 2.75 & 239.73 & 2.92 & 86.39 & 0.57 & 10.41 & 0.09 & 14.8 & 5.7 & 0.10 & 2.62 & 5308928 & 5309440 & & \\
\hline HD 71974 & 1889.06 & 13.60 & 1168.70 & 14.26 & 423.82 & 2.80 & 47.65 & 0.43 & 16.4 & 7.9 & 0.02 & 2.62 & 5393408 & 5393920 & & \\
\hline 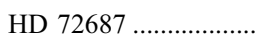 & 590.63 & 4.25 & 369.65 & 4.51 & 133.01 & 1.44 & 18.80 & 0.17 & 3.2 & 8.7 & 0.02 & 2.62 & 6600448 & 6598656 & & 2 \\
\hline HD $72905 \ldots \ldots \ldots \ldots \ldots$ & 6226.53 & 44.83 & 3915.21 & 47.77 & 1411.52 & 9.32 & 163.5 & 1.5 & 44.5 & 5.4 & 0.02 & 2.62 & 5362688 & 4042240 & & 4 \\
\hline 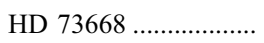 & 1401.43 & 10.09 & 881.16 & 10.75 & 313.64 & 2.07 & 35.39 & 0.32 & 10.5 & 10.4 & 0.02 & 2.62 & 5435392 & 5435904 & & \\
\hline 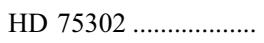 & 1315.76 & 9.47 & 819.18 & 9.99 & 293.72 & 1.94 & 32.70 & 0.29 & 1.4 & 8.6 & 0.02 & 2.62 & 5404160 & 5404672 & & \\
\hline 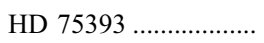 & 1244.85 & 8.96 & 787.10 & 9.60 & 281.09 & 1.85 & 31.70 & 0.29 & -6.2 & 9.0 & 0.02 & 2.62 & 5341184 & 5341696 & & \\
\hline HD $76218 \ldots \ldots \ldots \ldots \ldots$ & 1329.90 & 9.57 & 833.15 & 10.16 & 300.52 & 1.98 & 33.66 & 0.30 & -5.1 & 10.0 & 0.02 & 2.62 & 5373696 & 5374208 & & \\
\hline HD 77407 ........................ & 1910.85 & 13.76 & 1211.53 & 14.78 & 444.45 & 2.93 & 49.20 & 0.44 & 11.1 & 9.3 & 0.02 & 2.62 & 5311232 & 5311744 & & \\
\hline 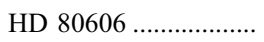 & 339.01 & 2.44 & 210.02 & 2.56 & 75.52 & 0.50 & 8.65 & 0.08 & 3.4 & 5.2 & 0.10 & 2.62 & 5443840 & 5444352 & & \\
\hline HD 85301 & 1050.80 & 7.57 & 652.06 & 7.96 & 234.22 & 2.30 & 36.80 & 0.33 & 38.5 & 7.0 & 0.02 & 2.62 & 5399552 & 5400064 & & \\
\hline HD 86356 & 186.44 & 1.34 & 117.14 & 1.43 & 42.97 & 0.28 & 5.12 & 0.05 & -2.6 & 4.3 & 0.10 & 9.96 & 5260032 & 5260544 & & \\
\hline 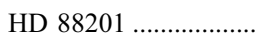 & 1022.26 & 7.36 & 644.98 & 7.87 & 229.89 & 1.52 & 25.62 & 0.23 & -1.2 & 7.3 & 0.02 & 2.62 & 5347328 & 5347840 & & \\
\hline
\end{tabular}


TABLE 1-Continued

\begin{tabular}{|c|c|c|c|c|c|c|c|c|c|c|c|c|c|c|c|c|}
\hline \multirow[b]{4}{*}{ Source } & \multicolumn{6}{|c|}{ IRAC } & \multicolumn{4}{|c|}{ MIPS } & \multirow{4}{*}{$\begin{array}{l}\text { IRAC FRAME } \\
\text { Time } \\
(\mathrm{s})\end{array}$} & \multirow{4}{*}{$\begin{array}{c}\text { MIPS } 24 \mu \mathrm{m} \\
\text { DCE TIME } \\
\text { (s) }\end{array}$} & \multirow{3}{*}{\multicolumn{2}{|c|}{ AOR KeY }} & \multirow{3}{*}{\multicolumn{2}{|c|}{ FlaGs $^{\mathrm{a}}$}} \\
\hline & \multicolumn{2}{|c|}{$3.6 \mu \mathrm{m}$} & \multicolumn{2}{|c|}{$4.5 \mu \mathrm{m}$} & \multicolumn{2}{|c|}{$8 \mu \mathrm{m}$} & \multicolumn{2}{|c|}{$24 \mu \mathrm{m}$} & \multicolumn{2}{|c|}{$70 \mu \mathrm{m}$} & & & & & & \\
\hline & \multirow{2}{*}{$\begin{array}{c}S_{\nu} \\
(\mathrm{mJy})\end{array}$} & \multirow{2}{*}{$\begin{array}{l}\sigma_{\mathrm{int}} \\
(\mathrm{mJy})\end{array}$} & \multirow{2}{*}{$\begin{array}{c}S_{\nu} \\
(\mathrm{mJy})\end{array}$} & \multirow{2}{*}{$\begin{array}{l}\sigma_{\mathrm{int}} \\
(\mathrm{mJy})\end{array}$} & \multirow{2}{*}{$\begin{array}{l}S_{\nu} \\
(\mathrm{mJy})\end{array}$} & & $S_{u}$ & & $S_{1}$ & & & & & & & \\
\hline & & & & & & (mJy) & (mJy) & (mJy) & (mJy) & (mJy) & & & IRAC & MIPS & IRAC & MIPS \\
\hline HD 88742 & 2966.24 & 21.36 & 1865.15 & 22.75 & 663.21 & 4.38 & 73.26 & 0.66 & 8.4 & 8.9 & 0.02 & 2.62 & 5422336 & 5422848 & & \\
\hline HD $90712 \ldots \ldots$ & 1043.36 & 7.51 & 666.66 & 8.13 & 235.39 & 1.55 & 26.13 & 0.24 & -2.7 & 8.0 & 0.02 & 2.62 & 5337344 & 5337856 & & \\
\hline HD 90905 & 1788.78 & 12.88 & 1126.18 & 13.74 & 401.52 & 2.65 & 49.73 & 0.45 & 22.3 & 11.4 & 0.02 & 2.62 & 5335808 & 5336320 & & \\
\hline 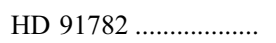 & 573.48 & 4.13 & 361.12 & 4.41 & 127.76 & 1.36 & 14.78 & 0.13 & -1.9 & 7.5 & 0.02 & 2.62 & 5331200 & 5331712 & & \\
\hline HD $91962 \ldots \ldots \ldots \ldots \ldots . .$. & 2018.84 & 14.54 & 1264.75 & 15.43 & 457.91 & 3.02 & 50.77 & 0.46 & -0.4 & 7.8 & 0.02 & 2.62 & 5341952 & 5342464 & 1 & \\
\hline 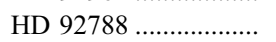 & 1447.46 & 10.42 & 891.20 & 10.87 & 322.72 & 2.13 & 36.08 & 0.32 & 11.2 & 9.1 & 0.02 & 2.62 & 5440000 & 5440512 & & \\
\hline 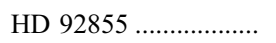 & 1280.32 & 9.22 & 810.92 & 9.89 & 288.82 & 1.91 & 32.43 & 0.29 & 9.8 & 6.8 & 0.02 & 2.62 & 5331968 & 5332480 & & \\
\hline 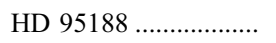 & 627.15 & 4.51 & 391.95 & 4.78 & 140.03 & 0.92 & 15.95 & 0.14 & 6.1 & 9.5 & 0.02 & 2.62 & 5344256 & 5344768 & & \\
\hline HD 98553 & 1067.94 & 7.69 & 669.07 & 8.16 & 238.75 & 1.58 & 26.35 & 0.24 & 3.9 & 8.0 & 0.02 & 2.62 & 5408000 & 5408512 & & \\
\hline HD $100167 \ldots \ldots \ldots \ldots \ldots . .$. & 1361.36 & 9.80 & 855.45 & 10.44 & 307.60 & 2.03 & 34.35 & 0.31 & -2.9 & 8.7 & 0.02 & 2.62 & 5420032 & 5420544 & & \\
\hline HD $101472 \ldots \ldots \ldots \ldots \ldots \ldots$ & 1036.87 & 7.46 & 665.02 & 8.11 & 233.44 & 1.77 & 26.19 & 0.24 & -0.6 & 8.8 & 0.02 & 2.62 & 5342720 & 5343232 & & \\
\hline HD $101959 \ldots \ldots \ldots \ldots$ & 1612.53 & 11.61 & 1013.24 & 12.36 & 361.27 & 2.38 & 40.18 & 0.36 & 9.8 & 8.4 & 0.02 & 2.62 & 5418496 & 5419008 & & \\
\hline HD 102071 & 1103.79 & 7.95 & 679.71 & 8.29 & 246.67 & 1.63 & 27.71 & 0.25 & 9.5 & 7.4 & 0.02 & 2.62 & 5423104 & 5423616 & & \\
\hline 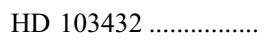 & 676.31 & 4.87 & 425.79 & 5.20 & 150.78 & 1.13 & 16.82 & 0.15 & 8.4 & 9.8 & 0.02 & 2.62 & 5428480 & 5428992 & & \\
\hline HD $104467 \ldots \ldots \ldots \ldots \ldots . . . .$. & 535.57 & 3.86 & 336.17 & 4.10 & 121.77 & 0.88 & 14.16 & 0.13 & -1.9 & 5.0 & 0.02 & 2.62 & 5208320 & 5208832 & & 2 \\
\hline 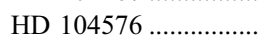 & 616.98 & 4.44 & 383.90 & 4.68 & 142.62 & 1.60 & 16.22 & 0.15 & 6.9 & 8.0 & 0.02 & 2.62 & 5332736 & 5333248 & & \\
\hline 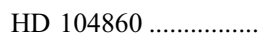 & 724.76 & 5.22 & 455.33 & 5.55 & 162.49 & 1.13 & 19.89 & 0.18 & 183.1 & 7.4 & 0.02 & 2.62 & 5270016 & 5270528 & & \\
\hline HD $105631 \ldots \ldots \ldots \ldots \ldots$ & 1613.93 & 11.62 & 989.25 & 12.07 & 360.21 & 2.38 & 40.27 & 0.36 & 1.0 & 7.4 & 0.02 & 2.62 & 5415424 & 5415936 & & \\
\hline HD $106156 \ldots \ldots \ldots \ldots \ldots$ & 1012.92 & 7.29 & 623.21 & 7.60 & 227.72 & 1.50 & 25.47 & 0.23 & 15.1 & 9.3 & 0.02 & 2.62 & 5423872 & 5424384 & & \\
\hline HD $106252 \ldots \ldots \ldots \ldots \ldots \ldots$ & 1200.09 & 8.64 & 746.45 & 9.11 & 270.92 & 1.79 & 30.58 & 0.28 & 16.5 & 9.4 & 0.02 & 2.62 & 5442304 & 5442816 & & \\
\hline HD $106772 \ldots \ldots \ldots \ldots$ & 992.21 & 7.14 & 630.40 & 7.69 & 232.27 & 1.85 & 26.40 & 0.24 & -4.6 & 6.8 & 0.02 & 2.62 & 5301504 & 5302016 & & \\
\hline HD 107146 & 1711.29 & 12.32 & 1074.76 & 13.11 & 384.40 & 2.54 & 59.76 & 0.54 & 669.1 & 9.6 & 0.02 & 2.62 & 5312000 & 5312512 & & \\
\hline HD 107441 & 254.97 & 1.84 & 161.86 & 1.98 & 58.42 & 0.39 & 7.24 & 0.07 & 1.6 & 7.2 & 0.10 & 2.62 & 5234432 & 5234944 & & \\
\hline HD 108799 & 3417.14 & 24.60 & 2165.72 & 26.42 & 767.08 & 5.06 & 85.26 & 0.77 & 5.0 & 10.2 & 0.02 & 2.62 & 5338112 & 5338624 & 1 & \\
\hline HD $108944 \ldots \ldots \ldots \ldots \ldots . .$. & 1136.76 & 8.19 & 727.64 & 8.88 & 256.94 & 1.70 & 28.88 & 0.26 & 1.7 & 8.4 & 0.02 & 2.62 & 5334272 & 5334784 & 1 & \\
\hline 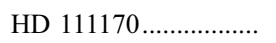 & 373.25 & 2.69 & 234.54 & 3.14 & 86.24 & 0.57 & 10.27 & 0.09 & -4.9 & 9.2 & 0.10 & 2.62 & 5213696 & 5214208 & & \\
\hline HD $112196 \ldots \ldots \ldots \ldots \ldots . . .$. & 1734.86 & 12.49 & 1098.91 & 13.41 & 395.59 & 2.61 & 43.62 & 0.39 & 3.2 & 9.1 & 0.02 & 2.62 & 5278464 & 5278976 & 1 & \\
\hline 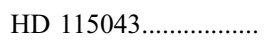 & 2122.18 & 15.28 & 1336.87 & 16.31 & 476.04 & 3.14 & 53.18 & 0.48 & -0.5 & 7.8 & 0.02 & 2.62 & 6600960 & 6599168 & & \\
\hline HD $116099 \ldots \ldots \ldots \ldots \ldots . . . .$. & 111.96 & 0.81 & 70.52 & 0.86 & 25.38 & 0.17 & 3.53 & 0.03 & 3.4 & 3.3 & 0.10 & 9.96 & 5229056 & 5229568 & & \\
\hline 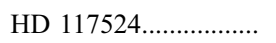 & 223.38 & 1.61 & 139.71 & 1.70 & 51.23 & 0.34 & 6.12 & 0.06 & -10.1 & 9.9 & 0.10 & 9.96 & 5231360 & 5231872 & & \\
\hline 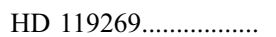 & 267.91 & 1.93 & 168.94 & 2.06 & 61.61 & 0.41 & 7.40 & 0.07 & 21.6 & 10.5 & 0.10 & 2.62 & 5239040 & 5239552 & & \\
\hline HD 120812 & 200.55 & 1.44 & 127.58 & 1.56 & 46.06 & 0.30 & 5.57 & 0.05 & -8.9 & 7.9 & 0.10 & 9.96 & 5227520 & 5228032 & & \\
\hline HD $121320 \ldots \ldots \ldots \ldots$ & 928.87 & 6.69 & 581.52 & 7.09 & 209.87 & 1.39 & 23.04 & 0.21 & 2.1 & 9.7 & 0.02 & 2.62 & 5424640 & 5425152 & & \\
\hline HD $121504 \ldots \ldots \ldots \ldots \ldots$ & 1001.83 & 7.21 & 630.62 & 7.69 & 225.25 & 1.49 & 25.01 & 0.23 & 27.2 & 18.7 & 0.02 & 2.62 & 5437696 & 5438208 & & \\
\hline 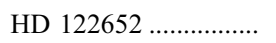 & 1260.59 & 9.08 & 795.28 & 9.70 & 283.09 & 1.87 & 35.22 & 0.32 & 83.1 & 9.1 & 0.02 & 2.62 & 5427712 & 5428224 & & \\
\hline HD $126670 \ldots \ldots \ldots \ldots . .$. & 216.52 & 1.56 & 137.21 & 1.67 & 49.79 & 0.33 & 6.17 & 0.07 & -0.8 & 5.7 & 0.10 & 2.62 & 5212928 & 5213440 & & \\
\hline HD $128242 \ldots \ldots \ldots \ldots \ldots \ldots$ & 224.73 & 1.62 & 143.13 & 1.75 & 52.02 & 0.34 & 6.32 & 0.06 & -6.3 & 5.8 & 0.10 & 9.96 & 5226752 & 5227264 & & \\
\hline HD 129333 & 1252.51 & 9.02 & 790.35 & 9.64 & 285.15 & 1.88 & 32.83 & 0.30 & 6.3 & 6.3 & 0.02 & 2.62 & 5265409 & 5265920 & & \\
\hline 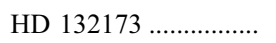 & 913.26 & 6.58 & 577.37 & 7.04 & 205.21 & 1.35 & 24.11 & 0.22 & -0.8 & 9.9 & 0.02 & 2.62 & 5333504 & 5334016 & & \\
\hline HD $133295 \ldots \ldots \ldots \ldots \ldots . .$. & 1343.76 & 9.68 & 852.62 & 10.40 & 301.06 & 1.99 & 34.10 & 0.31 & 12.2 & 12.4 & 0.02 & 2.62 & 5366784 & 5367296 & & \\
\hline HD $133938 \ldots \ldots \ldots \ldots$. & 128.42 & 0.93 & 81.78 & 1.00 & 29.60 & 0.20 & 3.48 & 0.03 & -5.0 & 11.5 & 0.10 & 9.96 & 5280768 & 5281280 & & \\
\hline HD 134319 & 548.74 & 3.95 & 339.31 & 4.14 & 121.37 & 0.80 & 15.41 & 0.14 & -3.4 & 8.1 & 0.02 & 2.62 & 5307392 & 5307904 & & \\
\hline HD $135363 \ldots \ldots \ldots \ldots \ldots . .$. & 1045.48 & 7.53 & 660.17 & 8.05 & 241.28 & 2.27 & 28.18 & 0.25 & 0.1 & 7.1 & 0.02 & 2.62 & 5293056 & 5293568 & & \\
\hline 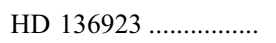 & 2175.65 & 15.66 & 1356.06 & 16.54 & 489.09 & 3.23 & 54.16 & 0.49 & 11.3 & 7.6 & 0.02 & 2.62 & 5429248 & 5429760 & & \\
\hline
\end{tabular}


TABLE 1-Continued

\begin{tabular}{|c|c|c|c|c|c|c|c|c|c|c|c|c|c|c|c|c|}
\hline \multirow[b]{4}{*}{ Source } & \multicolumn{6}{|c|}{ IRAC } & \multicolumn{4}{|c|}{ MIPS } & \multirow{4}{*}{$\begin{array}{l}\text { IRAC Frame } \\
\text { Time } \\
\text { (s) }\end{array}$} & \multirow{4}{*}{$\begin{array}{c}\text { MIPS } 24 \mu \mathrm{m} \\
\text { DCE TIME } \\
\text { (s) }\end{array}$} & \multirow{3}{*}{\multicolumn{2}{|c|}{ AOR KEY }} & \multirow{3}{*}{\multicolumn{2}{|c|}{ FlaGs $^{a}$}} \\
\hline & \multicolumn{2}{|c|}{$3.6 \mu \mathrm{m}$} & \multicolumn{2}{|c|}{$4.5 \mu \mathrm{m}$} & \multicolumn{2}{|c|}{$8 \mu \mathrm{m}$} & \multicolumn{2}{|c|}{$24 \mu \mathrm{m}$} & \multicolumn{2}{|c|}{$70 \mu \mathrm{m}$} & & & & & & \\
\hline & & & & & & & & & $S$ & & & & & & & \\
\hline & (mJy) & (mJy) & (mJy) & (mJy) & (mJy) & (mJy) & (mJy) & (mJy) & (mJy) & (mJy) & & & IRAC & MIPS & IRAC & MIPS \\
\hline HD $138004 \ldots \ldots \ldots \ldots \ldots \ldots . . .$. & 1208.71 & 8.70 & 749.81 & 9.15 & 269.34 & 1.78 & 29.76 & 0.27 & 1.6 & 7.3 & 0.02 & 2.62 & 5433088 & 5433600 & & \\
\hline HD $139498 \ldots \ldots \ldots \ldots \ldots \ldots . . . .$. & 287.36 & 2.07 & 181.71 & 2.22 & 66.48 & 0.44 & 7.89 & 0.10 & -7.4 & 15.7 & 0.10 & 2.62 & 5228288 & 5228800 & & \\
\hline 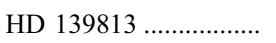 & 1843.07 & 13.27 & 1151.04 & 14.04 & 419.79 & 2.77 & 46.37 & 0.42 & 15.6 & 7.7 & 0.02 & 2.62 & 5336576 & 5337088 & & \\
\hline 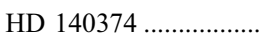 & 224.05 & 1.61 & 139.56 & 1.70 & 50.59 & 0.33 & 5.79 & 0.05 & -12.3 & 11.7 & 0.10 & 9.96 & 5224448 & 5224960 & & \\
\hline HD $141521 \ldots \ldots \ldots \ldots \ldots . .$. & 245.93 & 1.77 & 153.00 & 1.87 & 56.64 & 0.37 & 6.70 & 0.07 & -10.6 & 12.5 & 0.10 & 2.62 & 5225216 & 5225728 & & \\
\hline 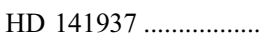 & 1392.65 & 10.03 & 872.31 & 10.64 & 310.76 & 2.05 & 34.94 & 0.31 & -2.8 & 11.6 & 0.02 & 2.62 & 5441536 & 5442048 & & \\
\hline 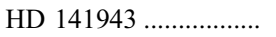 & 849.12 & 6.11 & 541.02 & 6.60 & 193.09 & 1.47 & 27.34 & 0.25 & 37.6 & 15.0 & 0.02 & 2.62 & 5252608 & 5253120 & & \\
\hline 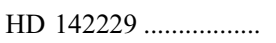 & 690.86 & 4.97 & 438.40 & 5.35 & 152.19 & 1.13 & 17.70 & 0.16 & 2.8 & 8.5 & 0.02 & 2.62 & 5384960 & 5385472 & & \\
\hline HD $142361 \ldots \ldots \ldots \ldots . . .$. & 459.81 & 3.31 & 292.68 & 3.57 & 107.66 & 0.80 & 12.74 & 0.11 & 12.2 & 19.4 & 0.02 & 2.62 & 5241344 & 5241856 & & \\
\hline HD $143006 \ldots \ldots \ldots \ldots \ldots . . .$. & 1069.37 & 7.70 & 929.86 & 11.34 & 792.11 & 5.23 & 3258 & 29 & 3795.1 & 33.3 & 0.02 & 2.62 & 5197312 & 5197824 & & \\
\hline HD $143358 \ldots \ldots \ldots \ldots \ldots \ldots . .$. & 173.57 & 1.25 & 111.01 & 1.35 & 40.14 & 0.28 & 5.22 & 0.05 & 2.1 & 14.8 & 0.10 & 9.96 & 5236736 & 5237248 & & \\
\hline 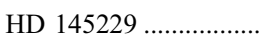 & 1128.81 & 8.13 & 717.36 & 8.75 & 254.00 & 1.68 & 31.02 & 0.28 & 64.4 & 7.3 & 0.02 & 2.62 & 5387264 & 5387776 & & \\
\hline 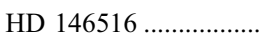 & 203.17 & 1.46 & 129.34 & 1.58 & 46.83 & 0.31 & 5.79 & 0.05 & 4.1 & 11.4 & 0.10 & 9.96 & 5218304 & 5218816 & & \\
\hline HD 150554 & 820.15 & 5.91 & 523.93 & 6.39 & 181.49 & 1.58 & 20.50 & 0.18 & -6.6 & 6.9 & 0.02 & 2.62 & 5443072 & 5443584 & & \\
\hline 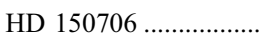 & 1715.11 & 12.35 & 1077.03 & 13.14 & 388.12 & 2.56 & 44.93 & 0.40 & 41.3 & 8.0 & 0.02 & 2.62 & 5385728 & 5386240 & & \\
\hline 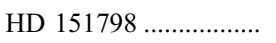 & 746.39 & 5.37 & 467.61 & 5.71 & 166.72 & 1.10 & 18.67 & 0.17 & -5.0 & 16.9 & 0.02 & 2.62 & 5276160 & 5276672 & & \\
\hline 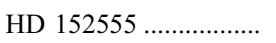 & 819.35 & 5.90 & 515.58 & 6.29 & 185.47 & 1.22 & 20.59 & 0.19 & -0.5 & 9.6 & 0.02 & 2.62 & 5330432 & 5330944 & 1 & \\
\hline 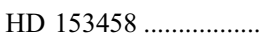 & 742.93 & 5.35 & 465.17 & 5.67 & 165.26 & 1.15 & 18.90 & 0.17 & -5.4 & 11.6 & 0.02 & 2.62 & 5416192 & 5416704 & & \\
\hline HD $154417 \ldots \ldots \ldots \ldots \ldots . .$. & 3993.62 & 28.75 & 2506.84 & 30.58 & 897.07 & 5.92 & 100.9 & 0.9 & 5.2 & 8.5 & 0.02 & 2.62 & 5398784 & 5399296 & & \\
\hline 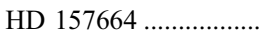 & 616.87 & 4.44 & 391.44 & 4.78 & 136.12 & 0.90 & 15.36 & 0.14 & 3.1 & 8.2 & 0.02 & 2.62 & 5445376 & 5445888 & & \\
\hline 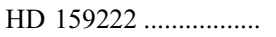 & 2730.18 & 19.66 & 1715.21 & 20.93 & 613.30 & 4.05 & 67.39 & 0.61 & 5.6 & 7.2 & 0.02 & 2.62 & 5436160 & 5436672 & & \\
\hline 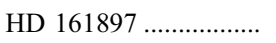 & 1217.95 & 8.77 & 754.95 & 9.21 & 273.67 & 1.81 & 30.18 & 0.27 & -1.8 & 11.6 & 0.02 & 2.62 & 5430016 & 5430528 & & \\
\hline HD $167389 \ldots \ldots \ldots \ldots . .$. & 1210.09 & 8.71 & 762.22 & 9.30 & 269.59 & 1.78 & 29.97 & 0.27 & -4.4 & 9.2 & 0.02 & 2.62 & 5433856 & 5434368 & & \\
\hline HD $170778 \ldots \ldots \ldots \ldots \ldots . .$. & 1076.58 & 7.75 & 681.74 & 8.32 & 241.21 & 1.59 & 27.23 & 0.25 & 3.9 & 6.3 & 0.02 & 2.62 & 5369856 & 5370368 & & \\
\hline 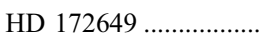 & 929.58 & 6.69 & 595.15 & 7.26 & 208.44 & 2.24 & 23.82 & 0.21 & -1.2 & 7.7 & 0.02 & 2.62 & 5335040 & 5335552 & & \\
\hline HD $174656 \ldots \ldots \ldots \ldots . .$. & 362.01 & 2.61 & 227.08 & 2.77 & 83.19 & 0.55 & 10.25 & 0.09 & 1.6 & 4.5 & 0.10 & 2.62 & 5195776 & 5196288 & & \\
\hline 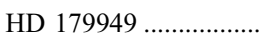 & 2942.99 & 21.19 & 1849.03 & 22.56 & 658.41 & 4.34 & 73.94 & 0.67 & -4.8 & 10.6 & 0.02 & 2.62 & 5440768 & 5441280 & & \\
\hline HD $183216 \ldots \ldots \ldots \ldots . .$. & 1430.42 & 10.30 & 896.03 & 10.93 & 320.78 & 2.12 & 39.41 & 0.35 & 22.8 & 10.2 & 0.02 & 2.62 & 5401856 & 5402368 & & \\
\hline 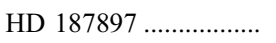 & 1495.45 & 10.77 & 934.10 & 11.40 & 338.68 & 2.23 & 39.79 & 0.36 & 61.6 & 8.2 & 0.02 & 2.62 & 5419264 & 5419776 & & \\
\hline 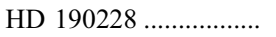 & 2068.09 & 14.89 & 1282.73 & 15.65 & 469.44 & 3.10 & 52.77 & 0.47 & 11.7 & 25.5 & 0.02 & 2.62 & 5438464 & 5438976 & & \\
\hline HD 191089 & 1071.72 & 7.72 & 678.37 & 8.28 & 242.17 & 1.60 & 185.6 & 1.7 & 544.3 & 12.5 & 0.02 & 2.62 & 5363968 & 5364224 & & \\
\hline 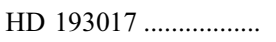 & 1179.47 & 8.49 & 743.73 & 9.07 & 264.71 & 1.75 & 29.90 & 0.27 & 6.6 & 9.3 & 0.02 & 2.62 & 5410048 & 5410560 & & \\
\hline HD 195034 & 1689.95 & 12.17 & 1060.98 & 12.94 & 379.42 & 2.50 & 41.94 & 0.38 & 1.6 & 8.4 & 0.02 & 2.62 & 5426176 & 5426688 & & \\
\hline 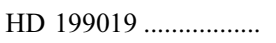 & 745.17 & 5.37 & 473.60 & 5.78 & 165.29 & 1.09 & 18.93 & 0.17 & 9.0 & 7.8 & 0.02 & 2.62 & 5343488 & 5344000 & & \\
\hline 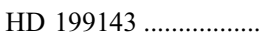 & 1401.22 & 10.09 & 899.64 & 10.98 & 320.68 & 2.42 & 37.60 & 0.34 & 9.2 & 11.1 & 0.02 & 2.62 & 5254144 & 5254656 & 1 & \\
\hline 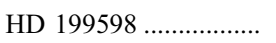 & 1843.53 & 13.27 & 1165.87 & 14.22 & 412.16 & 2.72 & 46.64 & 0.42 & 6.9 & 7.8 & 0.02 & 2.62 & 5413120 & 5413632 & & \\
\hline 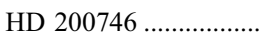 & 814.31 & 5.86 & 519.15 & 6.33 & 183.35 & 1.30 & 20.80 & 0.19 & 11.4 & 8.1 & 0.02 & 2.62 & 5371392 & 5371904 & & \\
\hline HD $201219 \ldots \ldots \ldots \ldots \ldots \ldots$ & 816.37 & 5.88 & 508.54 & 6.20 & 181.28 & 1.20 & 21.97 & 0.20 & 42.4 & 7.2 & 0.02 & 2.62 & 5396480 & 5396992 & & 2 \\
\hline 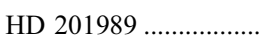 & 1440.45 & 10.37 & 902.15 & 11.01 & 323.35 & 2.13 & 36.16 & 0.33 & -0.2 & 9.5 & 0.02 & 2.62 & 5394944 & 5395456 & 1 & \\
\hline 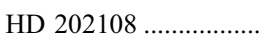 & 1440.33 & 10.37 & 902.09 & 11.01 & 321.78 & 2.12 & 35.98 & 0.32 & -0.3 & 9.6 & 0.02 & 2.62 & 5416960 & 5417472 & & \\
\hline 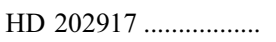 & 519.16 & 3.74 & 320.83 & 3.91 & 117.29 & 1.44 & 19.20 & 0.17 & 37.1 & 5.9 & 0.02 & 2.62 & 5251328 & 4558848 & & 4 \\
\hline HD $203030 \ldots \ldots \ldots \ldots . . .$. & 613.14 & 4.42 & 382.11 & 4.66 & 137.07 & 0.91 & 15.56 & 0.14 & 6.5 & 7.4 & 0.02 & 2.62 & 5338880 & 5339392 & & \\
\hline HD $204277 \ldots \ldots \ldots \ldots \ldots . . . .$. & 1847.54 & 13.30 & 1172.19 & 14.30 & 415.82 & 2.74 & 48.88 & 0.44 & 29.6 & 10.6 & 0.02 & 2.62 & 5374464 & 5374976 & & \\
\hline 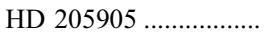 & 2172.60 & 15.64 & 1366.13 & 16.67 & 491.11 & 3.24 & 54.34 & 0.49 & 17.1 & 8.9 & 0.02 & 2.62 & 5404928 & 5405440 & & \\
\hline
\end{tabular}


TABLE 1-Continued

\begin{tabular}{|c|c|c|c|c|c|c|c|c|c|c|c|c|c|c|c|c|}
\hline \multirow[b]{4}{*}{ SOURCE } & \multicolumn{6}{|c|}{ IRAC } & \multicolumn{4}{|c|}{ MIPS } & \multirow{4}{*}{$\begin{array}{l}\text { IRAC FRAME } \\
\text { Time } \\
\text { (s) }\end{array}$} & \multirow{4}{*}{$\begin{array}{c}\text { MIPS } 24 \mu \mathrm{m} \\
\text { DCE TIME } \\
\text { (s) }\end{array}$} & \multirow{3}{*}{\multicolumn{2}{|c|}{ AOR KeY }} & \multirow{3}{*}{\multicolumn{2}{|c|}{ FLAGS $^{\mathrm{a}}$}} \\
\hline & \multicolumn{2}{|c|}{$3.6 \mu \mathrm{m}$} & \multicolumn{2}{|c|}{$4.5 \mu \mathrm{m}$} & \multicolumn{2}{|c|}{$8 \mu \mathrm{m}$} & \multicolumn{2}{|c|}{$24 \mu \mathrm{m}$} & \multicolumn{2}{|c|}{$70 \mu \mathrm{m}$} & & & & & & \\
\hline & \multirow{2}{*}{$\begin{array}{c}S_{\nu} \\
(\mathrm{mJy})\end{array}$} & \multirow{2}{*}{$\begin{array}{l}\sigma_{\mathrm{int}} \\
(\mathrm{mJy})\end{array}$} & \multirow{2}{*}{$\begin{array}{c}S_{\nu} \\
(\mathrm{mJy})\end{array}$} & \multirow{2}{*}{$\begin{array}{l}\sigma_{\mathrm{int}} \\
(\mathrm{mJy})\end{array}$} & \multirow{2}{*}{$\begin{array}{c}S_{\nu} \\
(\mathrm{mJy})\end{array}$} & & & & & & & & & & & \\
\hline & & & & & & (mJy) & (mJy) & $(\mathrm{mJy})$ & (mJy) & (mJy) & & & IRAC & MIPS & IRAC & MIPS \\
\hline 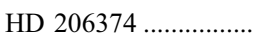 & 1389.08 & 10.00 & 871.06 & 10.63 & 312.08 & 2.06 & 35.22 & 0.32 & 18.1 & 6.7 & 0.02 & 2.62 & 5414656 & 5415168 & & \\
\hline HD $209253 \ldots \ldots \ldots \ldots \ldots . .$. & 2008.37 & 14.46 & 1285.28 & 15.68 & 454.49 & 3.00 & 55.94 & 0.50 & 75.0 & 9.2 & 0.02 & 2.62 & 5364480 & 5364992 & & \\
\hline HD $209393 \ldots \ldots \ldots \ldots \ldots . .$. & 856.52 & 6.17 & 531.63 & 6.49 & 192.30 & 1.84 & 21.74 & 0.20 & -2.4 & 9.0 & 0.02 & 2.62 & 5368320 & 5368832 & & \\
\hline HD 209779 & 1295.17 & 9.32 & 812.17 & 9.91 & 292.11 & 2.16 & 32.90 & 0.30 & 9.8 & 11.6 & 0.02 & 2.62 & 5369088 & 5369600 & & \\
\hline 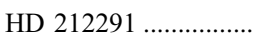 & 907.89 & 6.54 & 570.73 & 6.96 & 205.97 & 1.36 & 22.72 & 0.20 & 8.0 & 7.6 & 0.02 & 2.62 & 5420800 & 5421312 & & \\
\hline 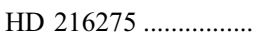 & 1379.27 & 9.93 & 867.30 & 10.58 & 309.52 & 2.04 & 34.54 & 0.31 & 7.3 & 10.5 & 0.02 & 2.62 & 5434624 & 5435136 & & 2 \\
\hline HD $216803 \ldots \ldots \ldots \ldots \ldots . . .$. & 8665.98 & 62.40 & 5318.78 & 64.89 & 1969.21 & 13.00 & 224.3 & 2.0 & 27.5 & 5.3 & 0.02 & 2.62 & 5255680 & 4058624 & & 4 \\
\hline HD $217343 \ldots \ldots \ldots \ldots \ldots$ & 1252.13 & 9.02 & 782.38 & 9.54 & 282.44 & 1.86 & 32.64 & 0.29 & -0.8 & 9.2 & 0.02 & 2.62 & 5269248 & 5269760 & & \\
\hline HD $219498 \ldots \ldots \ldots \ldots \ldots . .$. & 313.21 & 2.25 & 196.15 & 2.39 & 70.45 & 0.47 & 10.53 & 0.09 & 22.8 & 3.7 & 0.10 & 2.62 & 5357312 & 5357824 & & \\
\hline HD $224873 \ldots \ldots \ldots \ldots \ldots . . .$. & 625.86 & 4.51 & 388.50 & 4.74 & 140.78 & 0.93 & 15.50 & 0.14 & -6.7 & 8.5 & 0.02 & 2.62 & 5346560 & 5347072 & 1 & \\
\hline 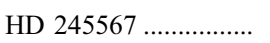 & 277.75 & 2.00 & 175.57 & 2.14 & 64.72 & 0.43 & 7.32 & 0.08 & -27.0 & 17.5 & 0.10 & 2.62 & 5248256 & 5248768 & & \\
\hline 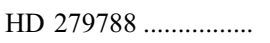 & 118.00 & 0.85 & 74.32 & 0.91 & 26.84 & 0.18 & 3.52 & 0.03 & 2.6 & 11.4 & 0.10 & 9.96 & 5245952 & 5246464 & & \\
\hline HD $281691 \ldots \ldots \ldots \ldots . .$. & 133.87 & 1.10 & 83.68 & 1.02 & 30.45 & 0.20 & 4.12 & 0.04 & -1.8 & 6.1 & 0.10 & 9.96 & 5259264 & 5259776 & & \\
\hline HD $282346 \ldots \ldots \ldots \ldots . . . .$. & 306.09 & 2.20 & 190.62 & 2.33 & 70.26 & 0.46 & 8.19 & 0.08 & -20.3 & 10.3 & 0.10 & 2.62 & 5303040 & 5303552 & & \\
\hline 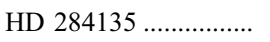 & 237.70 & 1.71 & 149.74 & 1.83 & 53.60 & 0.35 & 6.36 & 0.08 & 7.8 & 5.8 & 0.10 & 2.62 & 5206784 & 5207296 & & \\
\hline HD $284266 \ldots \ldots \ldots \ldots \ldots \ldots . .$. & 110.21 & 0.79 & 69.84 & 0.85 & 24.99 & 0.17 & 3.14 & 0.03 & -2.9 & 12.6 & 0.10 & 9.96 & 5220608 & 5221120 & & \\
\hline 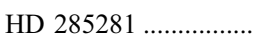 & 280.25 & 2.02 & 176.07 & 2.15 & 64.82 & 0.43 & 7.80 & 0.08 & -1.9 & 4.6 & 0.10 & 2.62 & 5216000 & 5216512 & & \\
\hline HD $285372 \ldots \ldots \ldots \ldots . . .$. & 93.54 & 0.67 & 58.77 & 0.72 & 21.50 & 0.17 & 2.55 & 0.03 & -4.5 & 9.4 & 0.10 & 9.96 & 5209856 & 5210368 & & \\
\hline 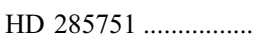 & 91.46 & 0.66 & 56.25 & 0.70 & 20.74 & 0.17 & 2.64 & 0.03 & -2.2 & 11.0 & 0.10 & 9.96 & 5207552 & 5208064 & & \\
\hline HD $285840 \ldots \ldots \ldots \ldots \ldots . .$. & 102.52 & 0.74 & 64.06 & 0.78 & 23.50 & 0.15 & 2.81 & 0.03 & -3.1 & 7.4 & 0.40 & 9.96 & 5359616 & 5360128 & & \\
\hline 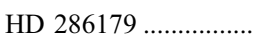 & 124.11 & 0.89 & 77.97 & 0.95 & 28.01 & 0.18 & 3.28 & 0.04 & -4.6 & 9.3 & 0.10 & 9.96 & 5221376 & 5221888 & & \\
\hline HD $286264 \ldots \ldots \ldots \ldots \ldots$ & 267.39 & 1.93 & 167.19 & 2.04 & 62.31 & 0.41 & 7.56 & 0.09 & -6.8 & 8.0 & 0.10 & 2.62 & 5250560 & 5251072 & & \\
\hline HE $350 \ldots \ldots \ldots \ldots \ldots \ldots \ldots . .$. & 56.95 & 0.41 & 36.36 & 0.44 & 13.10 & 0.09 & 1.63 & 0.02 & 6.0 & 9.8 & 0.40 & 9.96 & 5283072 & 5283584 & & \\
\hline 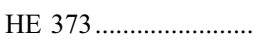 & 51.15 & 0.37 & 32.44 & 0.40 & 11.87 & 0.08 & 1.42 & 0.02 & -3.0 & 4.6 & 0.40 & 9.96 & 5283840 & 5284352 & & \\
\hline HE $389 \ldots \ldots \ldots \ldots \ldots \ldots \ldots . .$. & 46.64 & 0.34 & 29.26 & 0.36 & 10.55 & 0.07 & 1.22 & 0.02 & -0.1 & 10.4 & 0.40 & 9.96 & 5284608 & 5285120 & & \\
\hline 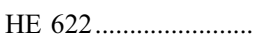 & 44.63 & 0.32 & 28.14 & 0.34 & 10.31 & 0.07 & 1.21 & 0.02 & 1.9 & 10.7 & 0.40 & 9.96 & 5285376 & 5285888 & & \\
\hline HE 696 & 39.98 & 0.29 & 25.79 & 0.32 & 9.12 & 0.06 & 1.07 & 0.02 & -20.2 & 11.0 & 0.40 & 9.96 & 5286144 & 5286656 & & \\
\hline HE 699 & 51.39 & 0.37 & 32.70 & 0.40 & 11.95 & 0.14 & 1.40 & 0.02 & 4.0 & 5.7 & 0.40 & 9.96 & 5286912 & 5287424 & & 2 \\
\hline HE $750 \ldots \ldots \ldots \ldots \ldots \ldots$ & 64.83 & 0.47 & 41.00 & 0.50 & 14.58 & 0.10 & 2.04 & 0.02 & -14.4 & 10.5 & 0.40 & 9.96 & 5287680 & 5288192 & & \\
\hline 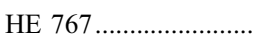 & 62.88 & 0.45 & 39.80 & 0.49 & 14.11 & 0.09 & 1.66 & 0.02 & -0.0 & 6.6 & 0.40 & 9.96 & 5288448 & 5288960 & & \\
\hline HE 848 & 115.60 & 0.83 & 72.57 & 0.89 & 25.57 & 0.23 & 4.60 & 0.04 & -7.9 & 11.7 & 0.10 & 9.96 & 5289216 & 5289728 & & \\
\hline HE 935 & 123.07 & 0.89 & 78.43 & 0.96 & 28.01 & 0.18 & 3.37 & 0.03 & -8.8 & 9.1 & 0.10 & 9.96 & 5289984 & 5290496 & & \\
\hline 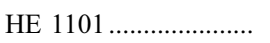 & 56.66 & 0.41 & 35.34 & 0.43 & 12.88 & 0.09 & 1.50 & 0.02 & -9.7 & 10.3 & 0.40 & 9.96 & 5290752 & 5291264 & & \\
\hline HE $1234 \ldots \ldots \ldots \ldots \ldots \ldots \ldots$ & 76.28 & 0.55 & 47.58 & 0.58 & 16.99 & 0.11 & 1.96 & 0.02 & -9.7 & 17.2 & 0.40 & 9.96 & 5291520 & 5292032 & & \\
\hline 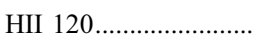 & 67.09 & 0.48 & 42.19 & 0.52 & 15.18 & 0.10 & 1.80 & 0.03 & -11.4 & 16.5 & 0.40 & 9.96 & 5327360 & 5327872 & & 2 \\
\hline 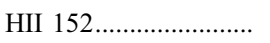 & 67.45 & 0.49 & 42.40 & 0.52 & 15.16 & 0.10 & 2.42 & 0.03 & 12.3 & 14.1 & 0.40 & 9.96 & 6601984 & 6600192 & & \\
\hline 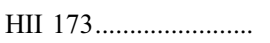 & 86.27 & 0.62 & 53.77 & 0.66 & 19.61 & 0.13 & 2.33 & 0.03 & -5.0 & 11.0 & 0.40 & 9.96 & 5315072 & 5315584 & & \\
\hline 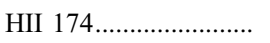 & 54.74 & 0.39 & 34.10 & 0.42 & 12.48 & 0.08 & 1.50 & 0.03 & 4.9 & 11.6 & 0.40 & 9.96 & 5315840 & 5316352 & & \\
\hline 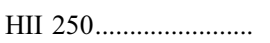 & 69.23 & 0.50 & 44.33 & 0.54 & 15.83 & 0.10 & 2.12 & 0.03 & 4.3 & 12.2 & 0.40 & 9.96 & 5316608 & 5317120 & & \\
\hline 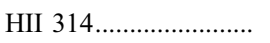 & 80.46 & 0.58 & 50.32 & 0.61 & 18.23 & 0.12 & 2.24 & 0.03 & 3.2 & 14.3 & 0.40 & 9.96 & 5314304 & 5314816 & & \\
\hline HII 514 & 70.16 & 0.51 & 44.24 & 0.54 & 15.99 & 0.11 & 2.34 & 0.03 & 9.8 & 9.3 & 0.40 & 9.96 & 5317376 & 5317888 & & \\
\hline HII $1015 \ldots \ldots \ldots \ldots \ldots \ldots . . .$. & 74.81 & 0.54 & 47.49 & 0.58 & 16.95 & 0.11 & 2.02 & 0.03 & -24.6 & 11.4 & 0.40 & 9.96 & 5318912 & 5319424 & & \\
\hline 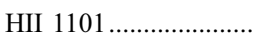 & 91.63 & 0.66 & 58.04 & 0.71 & 20.89 & 0.14 & 3.78 & 0.03 & 18.0 & 14.1 & 0.40 & 9.96 & 6601728 & 6599936 & & \\
\hline 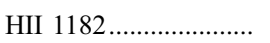 & 77.97 & 0.56 & 48.91 & 0.60 & 17.49 & 0.12 & 2.10 & 0.03 & -4.1 & 5.4 & 0.40 & 9.96 & 5319680 & 5320192 & & \\
\hline
\end{tabular}


TABLE 1-Continued

\begin{tabular}{|c|c|c|c|c|c|c|c|c|c|c|c|c|c|c|c|c|}
\hline \multirow[b]{4}{*}{ Source } & \multicolumn{6}{|c|}{ IRAC } & \multicolumn{4}{|c|}{ MIPS } & \multirow{4}{*}{$\begin{array}{c}\text { IRAC FRAME } \\
\text { Time } \\
\text { (s) }\end{array}$} & \multirow{4}{*}{$\begin{array}{c}\text { MIPS } 24 \mu \mathrm{m} \\
\text { DCE TIME } \\
\text { (s) }\end{array}$} & \multirow{3}{*}{\multicolumn{2}{|c|}{ AOR KEY }} & \multirow{3}{*}{\multicolumn{2}{|c|}{ FLAGS $^{\mathrm{a}}$}} \\
\hline & \multicolumn{2}{|c|}{$3.6 \mu \mathrm{m}$} & \multicolumn{2}{|c|}{$4.5 \mu \mathrm{m}$} & \multicolumn{2}{|c|}{$8 \mu \mathrm{m}$} & \multicolumn{2}{|c|}{$24 \mu \mathrm{m}$} & \multicolumn{2}{|c|}{$70 \mu \mathrm{m}$} & & & & & & \\
\hline & & & & & & & & & & & & & & & & \\
\hline & (mJy) & $(\mathrm{mJy})$ & (mJy) & $(\mathrm{mJy})$ & (mJy) & (mJy) & (mJy) & $(\mathrm{mJy})$ & $(\mathrm{mJy})$ & (mJy) & & & IRAC & MIPS & IRAC & MIPS \\
\hline HII $1200 \ldots \ldots$ & 112.26 & 0.81 & 71.86 & 0.88 & 25.02 & 0.17 & 3.43 & 0.03 & -22.2 & 15.6 & 0.10 & 9.96 & 5325824 & 5326336 & & \\
\hline HII 1776 & 62.92 & 0.45 & 39.69 & 0.48 & 14.42 & 0.10 & 1.75 & 0.03 & -6.1 & 11.1 & 0.40 & 9.96 & 5328128 & 5328640 & & \\
\hline HII $2147 \ldots \ldots \ldots$ & 108.24 & 0.78 & 67.89 & 0.83 & 24.55 & 0.17 & 2.98 & 0.03 & -4.5 & 5.7 & 0.10 & 9.96 & 5305344 & 5305856 & & \\
\hline HII $2278 \ldots \ldots \ldots \ldots \ldots \ldots \ldots \ldots \ldots \ldots \ldots$ & 91.16 & 0.66 & 56.95 & 0.69 & 20.87 & 0.14 & 2.49 & 0.03 & -14.9 & 9.9 & 0.40 & 9.96 & 5321216 & 5321728 & & \\
\hline HII $2506 \ldots \ldots \ldots \ldots \ldots$. & 85.60 & 0.62 & 54.11 & 0.66 & 19.23 & 0.13 & 2.33 & 0.03 & 5.7 & 4.6 & 0.40 & 9.96 & 5321984 & 5322496 & & \\
\hline HII 2644 & 54.39 & 0.39 & 33.97 & 0.41 & 12.22 & 0.08 & 1.43 & 0.03 & 3.8 & 6.6 & 0.40 & 9.96 & 5328896 & 5329408 & & \\
\hline HII 2786 & 83.50 & 0.64 & 53.36 & 0.65 & 18.84 & 0.12 & 2.20 & 0.03 & 2.0 & 9.8 & 0.40 & 9.96 & 5322752 & 5323264 & & \\
\hline HII $2881 \ldots \ldots \ldots \ldots$ & 68.28 & 0.49 & 43.24 & 0.53 & 15.74 & 0.10 & 1.78 & 0.03 & 8.1 & 5.9 & 0.40 & 9.96 & 5323520 & 5324032 & & \\
\hline HII 3097 & 67.41 & 0.48 & 42.31 & 0.52 & 15.13 & 0.10 & 1.84 & 0.03 & -5.7 & 4.9 & 0.40 & 9.96 & 5324288 & 5324800 & & \\
\hline HII 3179 & 101.55 & 0.73 & 64.27 & 0.78 & 22.48 & 0.15 & 2.70 & 0.03 & -3.7 & 3.9 & 0.10 & 9.96 & 5325056 & 5325568 & & \\
\hline HIP 6276 & 676.70 & 4.87 & 418.39 & 5.10 & 154.80 & 1.79 & 19.26 & 0.17 & 13.7 & 10.6 & 0.02 & 2.62 & 5345792 & 5346304 & & \\
\hline HIP $42491 \ldots \ldots$ & 705.78 & 5.08 & 431.44 & 5.26 & 157.50 & 1.53 & 17.63 & 0.16 & -8.1 & 11.9 & 0.02 & 2.62 & 5409536 & 5435904 & & \\
\hline HIP 59154 & 484.75 & 3.49 & 294.26 & 3.59 & 110.98 & 0.99 & 12.92 & 0.12 & 0.9 & 6.0 & 0.02 & 2.62 & 5360384 & 5360896 & & \\
\hline HIP 76477 & 154.54 & 1.11 & 96.58 & 1.18 & 35.86 & 0.24 & 4.35 & 0.04 & -0.9 & 8.8 & 0.10 & 9.96 & 5210624 & 5211136 & & \\
\hline MML 1 & 228.93 & 1.65 & 142.01 & 1.73 & 52.77 & 0.35 & 6.64 & 0.07 & 3.3 & 6.1 & 0.10 & 2.62 & 5240576 & 5241088 & & \\
\hline 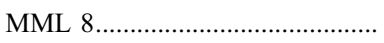 & 167.52 & 1.21 & 104.59 & 1.28 & 38.44 & 0.25 & 7.81 & 0.07 & 17.8 & 12.9 & 0.10 & 9.96 & 5238272 & 5238784 & & \\
\hline 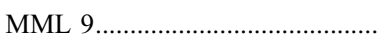 & 165.99 & 1.20 & 103.17 & 1.26 & 37.89 & 0.25 & 4.79 & 0.04 & -6.2 & 6.7 & 0.10 & 9.96 & 5237504 & 5238016 & & \\
\hline 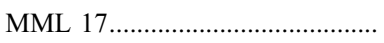 & 228.11 & 1.64 & 145.00 & 1.77 & 52.24 & 0.34 & 9.85 & 0.09 & 18.0 & 7.6 & 0.10 & 2.62 & 5232128 & 5232640 & & \\
\hline MML 18.................................. & 147.31 & 1.06 & 91.80 & 1.12 & 33.81 & 0.22 & 3.95 & 0.04 & -7.4 & 11.4 & 0.10 & 9.96 & 5277696 & 5278208 & & \\
\hline 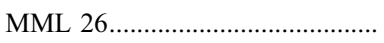 & 138.07 & 0.99 & 86.42 & 1.05 & 31.47 & 0.24 & 3.72 & 0.03 & -1.6 & 8.1 & 0.10 & 9.96 & 5230592 & 5231104 & 1 & \\
\hline 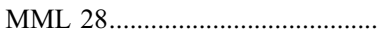 & 92.92 & 0.67 & 57.95 & 0.71 & 21.45 & 0.14 & 3.55 & 0.03 & 10.1 & 10.1 & 0.40 & 9.96 & 5276928 & 5277440 & & \\
\hline MML 32 & 132.67 & 0.95 & 84.46 & 1.03 & 30.41 & 0.20 & 3.72 & 0.03 & 6.4 & 12.0 & 0.10 & 9.96 & 5235968 & 5236480 & & \\
\hline 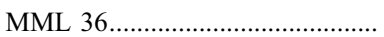 & 216.06 & 1.56 & 135.16 & 1.79 & 49.64 & 0.33 & 8.95 & 0.08 & 3.9 & 4.1 & 0.10 & 9.96 & 5235200 & 5235712 & & \\
\hline 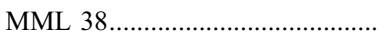 & 108.23 & 0.78 & 68.47 & 0.83 & 24.83 & 0.16 & 3.12 & 0.03 & -1.0 & 5.9 & 0.10 & 9.96 & 5233664 & 5234176 & & \\
\hline 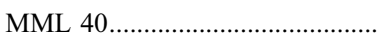 & 124.46 & 0.90 & 78.01 & 0.95 & 28.80 & 0.19 & 3.48 & 0.03 & -3.2 & 4.5 & 0.10 & 9.96 & 5239808 & 5240320 & & \\
\hline 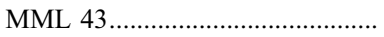 & 116.39 & 0.84 & 73.09 & 0.89 & 26.79 & 0.18 & 3.62 & 0.03 & -7.6 & 8.0 & 0.10 & 9.96 & 5232896 & 5233408 & & \\
\hline 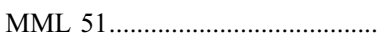 & 146.14 & 1.05 & 92.99 & 1.14 & 34.52 & 0.23 & 4.17 & 0.04 & 2.0 & 3.7 & 0.10 & 9.96 & 5212160 & 5212672 & & \\
\hline 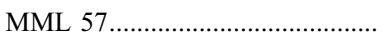 & 118.05 & 0.85 & 74.25 & 0.91 & 26.93 & 0.18 & 3.55 & 0.03 & 7.7 & 12.7 & 0.10 & 9.96 & 5229824 & 5230336 & & \\
\hline PDS 66 & 656.79 & 4.73 & 521.43 & 6.36 & 470.62 & 3.11 & 1874 & 94 & 1672.0 & 14.9 & 0.02 & 2.62 & 5198080 & 5198592 & & \\
\hline [PZ99] J155847.8-175800......... & 147.12 & 1.06 & 93.15 & 1.14 & 35.28 & 0.23 & 6.11 & 0.06 & -21.8 & 14.8 & 0.40 & 9.96 & 5202944 & 5203456 & & \\
\hline [PZ99] J160814.7-190833 ............. & 135.31 & 0.97 & 84.71 & 1.03 & 31.66 & 0.23 & 3.74 & 0.04 & 6.4 & 13.2 & 0.10 & 9.96 & 5249792 & 5250304 & & \\
\hline [PZ99] J161318.6-221248 ......... & 325.68 & 2.35 & 204.71 & 2.50 & 76.83 & 0.51 & 9.14 & 0.09 & -20.8 & 12.1 & 0.10 & 2.62 & 5203712 & 5204224 & & \\
\hline [PZ99] J161329.3-231106 ........ & 139.25 & 1.00 & 87.83 & 1.07 & 33.38 & 0.23 & 4.12 & 0.04 & 12.7 & 11.1 & 0.10 & 9.96 & 5204480 & 5204992 & 1 & \\
\hline [PZ99] J161402.1-230101 ......... & 119.64 & 0.86 & 75.34 & 0.92 & 28.25 & 0.19 & 3.66 & 0.04 & -19.6 & 13.0 & 0.10 & 9.96 & 5205248 & 5205760 & & \\
\hline [PZ99] J161411.0-230536 ......... & 498.31 & 3.59 & 401.87 & 4.90 & 363.52 & 2.40 & 304.0 & 2.7 & 91.1 & 11.7 & 0.02 & 2.62 & 5206016 & 5206528 & & \\
\hline [PZ99] J161459.2-275023 ......... & 101.69 & 0.73 & 63.27 & 0.77 & 23.80 & 0.16 & 4.53 & 0.04 & 26.6 & 25.0 & 0.10 & 9.96 & 5219072 & 5219584 & & \\
\hline [PZ99] J161618.0-233947 ......... & 170.49 & 1.23 & 106.52 & 1.30 & 40.09 & 0.27 & 5.35 & 0.05 & -14.8 & 14.0 & 0.10 & 9.96 & 5219840 & 5220352 & & \\
\hline 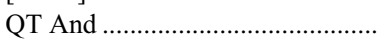 & 324.14 & 2.33 & 204.36 & 2.49 & 76.23 & 0.50 & 9.12 & 0.08 & -8.4 & 5.8 & 0.10 & 2.62 & 5299200 & 5299712 & & 2 \\
\hline R3 & 58.19 & 0.42 & 36.27 & 0.59 & 13.34 & 0.09 & 1.52 & 0.02 & -10.5 & 9.0 & 0.40 & 9.96 & 5272320 & 5272832 & & \\
\hline 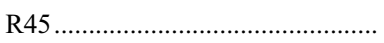 & 63.91 & 0.46 & 40.38 & 0.49 & 14.43 & 0.10 & 1.90 & 0.02 & 2.3 & 21.4 & 0.40 & 9.96 & 5273856 & 5274368 & & 2 \\
\hline 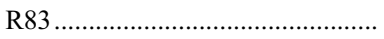 & 68.66 & 0.49 & 43.22 & 0.53 & 15.61 & 0.10 & 1.80 & 0.02 & -24.7 & 12.8 & 0.40 & 9.96 & 5273088 & 5273600 & & \\
\hline RE J0137+18A & 662.72 & 9.43 & 413.67 & 5.05 & 151.77 & 1.00 & 17.50 & 0.16 & 2.7 & 4.5 & 0.02 & 2.62 & 5242112 & 5242624 & 1 & \\
\hline 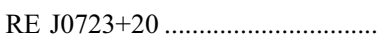 & 545.19 & 3.92 & 338.81 & 4.13 & 125.47 & 0.83 & 15.26 & 0.14 & 1.0 & 5.0 & 0.02 & 2.62 & 5348096 & 5348608 & & \\
\hline RX J0258.4+2947 ……................... & 71.67 & 0.52 & 44.92 & 0.55 & 16.40 & 0.11 & 1.92 & 0.02 & -5.3 & 4.0 & 0.40 & 9.96 & 5358080 & 5358592 & & \\
\hline
\end{tabular}


TABLE 1-Continued

\begin{tabular}{|c|c|c|c|c|c|c|c|c|c|c|c|c|c|c|c|c|}
\hline \multirow[b]{4}{*}{ SOURCE } & \multicolumn{6}{|c|}{ IRAC } & \multicolumn{4}{|c|}{ MIPS } & \multirow{4}{*}{$\begin{array}{l}\text { IRAC FraME } \\
\text { Time } \\
\text { (s) }\end{array}$} & \multirow{4}{*}{$\begin{array}{c}\text { MIPS } 24 \mu \mathrm{m} \\
\text { DCE TIME } \\
\text { (s) }\end{array}$} & \multirow{3}{*}{\multicolumn{2}{|c|}{ AOR KEY }} & \multirow{3}{*}{\multicolumn{2}{|c|}{ FlaGS $^{a}$}} \\
\hline & \multicolumn{2}{|c|}{$3.6 \mu \mathrm{m}$} & \multicolumn{2}{|c|}{$4.5 \mu \mathrm{m}$} & \multicolumn{2}{|c|}{$8 \mu \mathrm{m}$} & \multicolumn{2}{|c|}{$24 \mu \mathrm{m}$} & \multicolumn{2}{|c|}{$70 \mu \mathrm{m}$} & & & & & & \\
\hline & \multirow{2}{*}{$\begin{array}{c}S_{\nu} \\
\text { (mJy) }\end{array}$} & \multirow{2}{*}{$\begin{array}{l}\sigma_{\text {int }} \\
(\mathrm{mJy})\end{array}$} & \multirow{2}{*}{$\begin{array}{c}S_{\nu} \\
(\mathrm{mJy})\end{array}$} & \multirow{2}{*}{$\begin{array}{l}\sigma_{\text {int }} \\
(\mathrm{mJy})\end{array}$} & \multirow{2}{*}{$\begin{array}{c}S_{\nu} \\
\text { (mJy) }\end{array}$} & & & & $S_{1}$ & & & & & & & \\
\hline & & & & & & (mJy) & (mJy) & (mJy) & (mJy) & (mJy) & & & IRAC & MIPS & IRAC & MIPS \\
\hline RX J0329.1+0118 .............. & 63.93 & 0.46 & 40.80 & 0.50 & 14.68 & 0.10 & 1.71 & 0.02 & 2.1 & 4.8 & 0.40 & 9.96 & 5352704 & 5353216 & 1 & \\
\hline RX J0331.1+0713 ............. & 179.78 & 1.29 & 112.98 & 1.38 & 42.16 & 0.28 & 4.94 & 0.04 & -4.7 & 5.3 & 0.10 & 9.96 & 5242880 & 5243392 & 1 & \\
\hline RX J0354.4+0535 ............. & 103.00 & 0.74 & 65.35 & 0.80 & 23.61 & 0.18 & 3.09 & 0.03 & -7.2 & 5.0 & 0.10 & 9.96 & 5299968 & 5300480 & & \\
\hline RX J0357.3+1258 .............. & 77.05 & 0.56 & 48.40 & 0.59 & 17.72 & 0.12 & 2.20 & 0.03 & -3.3 & 5.7 & 0.40 & 9.96 & 5256192 & 5256704 & & \\
\hline RX J0434.3+0226............. & 48.51 & 0.35 & 31.07 & 0.38 & 11.46 & 0.08 & 1.38 & 0.02 & -5.9 & 4.1 & 0.40 & 9.96 & 5300736 & 5301248 & 1 & 2 \\
\hline RX J0442.5+0906............. & 67.02 & 0.48 & 41.52 & 0.51 & 15.23 & 0.10 & 1.75 & 0.03 & 1.2 & 4.5 & 0.40 & 9.96 & 5304576 & 5305088 & & \\
\hline RX J0849.2-7735 …........ & 1444.48 & 10.40 & 881.70 & 10.76 & 336.69 & 2.22 & 39.20 & 0.35 & 3.1 & 8.0 & 0.02 & 2.62 & 5312768 & 5313280 & & \\
\hline RX J0850.1-7554 ........... & 98.81 & 0.71 & 61.45 & 0.75 & 22.27 & 0.15 & 2.78 & 0.03 & 3.3 & 3.5 & 0.10 & 9.96 & 5298432 & 5298944 & & \\
\hline RX J0853.1-8244 „........... & 72.44 & 0.52 & 45.83 & 0.56 & 17.03 & 0.11 & 2.05 & 0.02 & -5.3 & 3.3 & 0.40 & 9.96 & 5351168 & 5351680 & & \\
\hline RX J0917.2-7744 ............ & 91.21 & 0.66 & 57.60 & 0.70 & 20.83 & 0.14 & 2.38 & 0.02 & 3.1 & 3.3 & 0.40 & 9.96 & 5351936 & 5352448 & & 2 \\
\hline RX J1111.7-7620 ............ & 447.93 & 3.23 & 363.46 & 4.43 & 198.69 & 1.86 & 229.6 & 2.1 & 224.3 & 8.3 & 0.02 & 2.62 & 5199616 & 5200128 & & 2 \\
\hline RX J1140.3-8321 ............ & 114.19 & 0.82 & 69.97 & 0.85 & 26.43 & 0.17 & 3.02 & 0.03 & -2.0 & 5.0 & 0.10 & 9.96 & 5260800 & 5261312 & & \\
\hline RX J1203.7-8129 ............ & 71.75 & 0.52 & 45.29 & 0.55 & 16.61 & 0.11 & 1.99 & 0.02 & 2.6 & 4.7 & 0.40 & 9.96 & 5354240 & 5354752 & & \\
\hline RX J1209.8-7344 …........ & 163.91 & 1.18 & 102.40 & 1.25 & 38.64 & 0.26 & 4.62 & 0.04 & -4.4 & 5.0 & 0.10 & 9.96 & 5361152 & 5361664 & & \\
\hline RX J1220.6-7539 ............ & 200.62 & 1.45 & 124.54 & 1.52 & 46.07 & 0.30 & 5.48 & 0.05 & -12.4 & 4.3 & 0.10 & 9.96 & 5355008 & 5355520 & & 2 \\
\hline RX J1225.3-7857 ............ & 112.89 & 0.81 & 70.60 & 0.86 & 25.73 & 0.17 & 3.05 & 0.03 & -0.9 & 3.9 & 0.10 & 9.96 & 5355776 & 5356288 & & \\
\hline RX J1450.4-3507 ............. & 178.29 & 1.28 & 112.17 & 1.37 & 41.31 & 0.27 & 4.91 & 0.04 & -3.7 & 4.0 & 0.10 & 9.96 & 5214464 & 5214976 & & \\
\hline RX J1457.3-3613 …......... & 149.56 & 1.08 & 94.73 & 1.16 & 34.38 & 0.23 & 4.32 & 0.04 & -1.2 & 5.4 & 0.10 & 9.96 & 5225984 & 5226496 & & \\
\hline RX J1458.6-3541 ................... & 211.96 & 1.53 & 133.68 & 1.63 & 49.73 & 0.33 & 5.97 & 0.05 & 7.6 & 4.2 & 0.10 & 9.96 & 5211392 & 5211904 & & \\
\hline RX J1500.8-4331 ............ & 96.40 & 0.69 & 61.33 & 0.75 & 22.54 & 0.15 & 2.65 & 0.02 & -10.0 & 8.2 & 0.40 & 9.96 & 5264640 & 5265152 & & \\
\hline RX J1507.2-3505 ............. & 137.35 & 0.99 & 85.39 & 1.04 & 31.64 & 0.21 & 4.05 & 0.04 & -0.1 & 6.1 & 0.10 & 9.96 & 5270784 & 5271296 & & \\
\hline RX J1518.4-3738 ............ & 121.24 & 0.87 & 77.05 & 0.94 & 28.29 & 0.19 & 3.48 & 0.04 & -1.8 & 5.8 & 0.10 & 9.96 & 5281536 & 5282048 & & \\
\hline RX J1531.3-3329 _........ & 95.38 & 0.69 & 59.77 & 0.73 & 21.97 & 0.14 & 2.49 & 0.04 & 12.4 & 13.1 & 0.40 & 9.96 & 5280000 & 5280512 & & \\
\hline RX J1541.1-2656 ............ & 82.17 & 0.59 & 51.08 & 0.62 & 19.05 & 0.13 & 2.27 & 0.03 & -37.2 & 13.3 & 0.40 & 9.96 & 5202176 & 5202688 & & \\
\hline RX J1544.0-3311 …........ & 138.34 & 1.00 & 87.01 & 1.06 & 32.21 & 0.21 & 4.04 & 0.04 & -22.4 & 12.0 & 0.10 & 9.96 & 5279232 & 5279744 & 1 & \\
\hline RX J1545.9-4222 …........ & 209.09 & 1.50 & 131.65 & 1.61 & 49.24 & 0.33 & 5.83 & 0.07 & -27.9 & 12.6 & 0.10 & 2.62 & 5215232 & 5215744 & & \\
\hline RX J1600.6-2159 ............. & 134.55 & 0.97 & 85.17 & 1.04 & 31.42 & 0.21 & 4.38 & 0.04 & 9.9 & 15.7 & 0.10 & 9.96 & 5253376 & 5253888 & & \\
\hline RX J1839.0-3726 ........... & 112.27 & 0.81 & 70.86 & 0.86 & 25.95 & 0.17 & 3.39 & 0.04 & -7.6 & 7.2 & 0.10 & 9.96 & 5223680 & 5224192 & & \\
\hline RX J1841.8-3525 ............. & 187.75 & 1.35 & 119.45 & 1.46 & 43.02 & 0.28 & 5.37 & 0.05 & -1.7 & 5.3 & 0.10 & 9.96 & 5216768 & 5217280 & & \\
\hline RX J1842.9-3532 ............ & 269.53 & 1.94 & 216.15 & 2.64 & 157.35 & 1.04 & 358.9 & 3.2 & 942.6 & 13.8 & 0.10 & 2.62 & 5198848 & 5199360 & & \\
\hline RX J1844.3-3541 …........ & 141.52 & 1.02 & 88.66 & 1.08 & 32.65 & 0.22 & 3.84 & 0.04 & -17.9 & 6.5 & 0.10 & 9.96 & 5249024 & 5249536 & & \\
\hline RX J1852.3-3700 ............ & 88.58 & 0.64 & 58.49 & 2.04 & 33.64 & 0.97 & 472.2 & 4.2 & 1367.0 & 14.6 & 0.02 & 2.62 & 5200384 & 5200896 & & \\
\hline RX J1917.4-3756 ............ & 318.64 & 2.29 & 198.72 & 2.42 & 73.67 & 0.49 & 8.82 & 0.08 & 2.1 & 6.9 & 0.10 & 2.62 & 5209088 & 5209600 & & \\
\hline RX J2313.0+2345 ............... & 107.93 & 0.78 & 67.33 & 0.82 & 24.13 & 0.23 & 2.80 & 0.03 & -3.3 & 4.4 & 0.10 & 9.96 & 5243648 & 5244160 & 1 & 2 \\
\hline 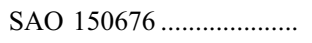 & 306.12 & 2.20 & 191.84 & 2.34 & 70.27 & 0.46 & 8.72 & 0.08 & 2.1 & 4.3 & 0.10 & 2.62 & 5294592 & 5295104 & & \\
\hline 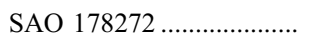 & 327.04 & 2.35 & 203.57 & 2.48 & 75.43 & 0.50 & 9.08 & 0.08 & 1.5 & 3.5 & 0.10 & 2.62 & 5309696 & 5310208 & & \\
\hline ScoPMS 21 ........................... & 120.69 & 0.87 & 76.08 & 0.93 & 28.71 & 0.19 & 3.62 & 0.06 & -8.1 & 26.1 & 0.10 & 9.96 & 5201152 & 5201664 & & \\
\hline 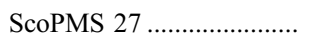 & 186.26 & 1.34 & 116.92 & 1.43 & 43.94 & 0.29 & 5.25 & 0.08 & 7.3 & 11.4 & 0.10 & 2.62 & 5196544 & 5197056 & & \\
\hline ScoPMS $52 \ldots \ldots \ldots \ldots \ldots \ldots$. & 319.90 & 2.30 & 200.77 & 2.45 & 75.93 & 0.50 & 8.89 & 0.11 & 34.5 & 23.8 & 0.10 & 2.62 & 5217536 & 5218048 & & \\
\hline 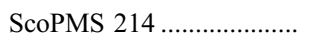 & 242.00 & 1.74 & 154.41 & 1.88 & 59.07 & 0.39 & 7.75 & 0.14 & 15.1 & 15.4 & 0.60 & 2.62 & 5672448 & 5706496 & 3 & 4 \\
\hline 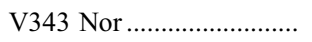 & 1344.03 & 9.68 & 846.43 & 10.33 & 308.70 & 2.04 & 36.77 & 0.33 & -1.3 & 40.0 & 0.02 & 2.62 & 5222912 & 5223424 & & \\
\hline V383 Lac ............................. & 752.12 & 5.42 & 466.84 & 5.70 & 170.11 & 1.33 & 20.27 & 0.18 & 7.8 & 8.6 & 0.02 & 2.62 & 5296128 & 5296640 & & \\
\hline 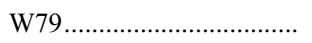 & 42.02 & 0.30 & 26.01 & 0.32 & 9.52 & 0.06 & 1.38 & 0.02 & 0.2 & 13.7 & 0.40 & 9.96 & 5274624 & 5275136 & & \\
\hline vB 1 & 1157.67 & 8.34 & 733.56 & 8.95 & 259.47 & 1.71 & 28.51 & 0.26 & 14.1 & 12.2 & 0.02 & 2.62 & 5403392 & 5403904 & 1 & \\
\hline
\end{tabular}


TABLE 1-Continued

\begin{tabular}{|c|c|c|c|c|c|c|c|c|c|c|c|c|c|c|c|c|}
\hline \multirow[b]{4}{*}{ Source } & \multicolumn{6}{|c|}{ IRAC } & \multicolumn{4}{|c|}{ MIPS } & \multirow{4}{*}{$\begin{array}{c}\text { IRAC Frame } \\
\text { Time } \\
\text { (s) }\end{array}$} & \multirow{4}{*}{$\begin{array}{c}\text { MIPS } 24 \mu \mathrm{m} \\
\text { DCE TIME } \\
\text { (s) }\end{array}$} & \multirow{3}{*}{\multicolumn{2}{|c|}{ AOR Key }} & \multirow{3}{*}{\multicolumn{2}{|c|}{ FLAGS $^{a}$}} \\
\hline & \multicolumn{2}{|c|}{$3.6 \mu \mathrm{m}$} & \multicolumn{2}{|c|}{$4.5 \mu \mathrm{m}$} & \multicolumn{2}{|c|}{$8 \mu \mathrm{m}$} & \multicolumn{2}{|c|}{$24 \mu \mathrm{m}$} & \multicolumn{2}{|c|}{$70 \mu \mathrm{m}$} & & & & & & \\
\hline & $S$ & & $S$ & & $S$ & & $S$ & & $S$ & & & & & & & \\
\hline & (mJy) & (mJy) & (mJy) & (mJy) & (mJy) & (mJy) & (mJy) & (mJy) & (mJy) & (mJy) & & & IRAC & MIPS & IRAC & MIPS \\
\hline vB 39 & 907.63 & 6.54 & 581.32 & 7.09 & 214.07 & 1.95 & 24.26 & 0.22 & -3.5 & 10.3 & 0.60 & 2.62 & 4096512 & 5376256 & 3 & \\
\hline vB 49 & 531.47 & 3.83 & 333.05 & 5.62 & 123.24 & 1.12 & 13.61 & 0.12 & -5.8 & 7.7 & 0.60 & 2.62 & 4096512 & 5376768 & 3 & \\
\hline 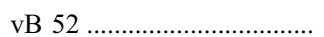 & 856.95 & 6.17 & 545.70 & 6.66 & 200.27 & 1.32 & 22.48 & 0.20 & 0.6 & 10.6 & 0.60 & 2.62 & 4096512 & 5377280 & 3 & \\
\hline vB 63 & 750.87 & 6.02 & 480.71 & 5.87 & 177.66 & 1.52 & 19.97 & 0.18 & 3.0 & 10.7 & 0.60 & 2.62 & 4096512 & 5377792 & 3 & \\
\hline vB 64 & 658.77 & 4.78 & 422.85 & 5.16 & 152.25 & 1.00 & 16.96 & 0.15 & -4.3 & 11.8 & 0.60 & 2.62 & 4096512 & 5378304 & 3 & \\
\hline vB 66 & 961.97 & 6.93 & 609.26 & 7.43 & 216.22 & 1.43 & 24.58 & 0.22 & 10.2 & 11.4 & 0.02 & 2.62 & 5367552 & 5368064 & & \\
\hline vB 73 & 756.94 & 5.45 & 476.39 & 5.81 & 176.50 & 1.17 & 20.16 & 0.18 & -8.4 & 10.4 & 0.60 & 2.62 & 4096512 & 5378816 & 3 & \\
\hline vB 79 & 422.74 & 3.14 & 266.31 & 4.12 & 99.03 & 1.03 & 10.95 & 0.10 & -7.6 & 7.9 & 0.60 & 2.62 & 4096512 & 5379328 & 3 & \\
\hline vB 88 & 741.40 & 5.34 & 469.92 & 5.73 & 165.43 & 1.09 & 18.93 & 0.17 & -11.3 & 12.5 & 0.02 & 2.62 & 5397248 & 5397760 & & \\
\hline vB 91 & 574.70 & 4.14 & 349.43 & 4.26 & 131.46 & 0.87 & 14.81 & 0.13 & -9.3 & 12.7 & 0.60 & 2.62 & 4096768 & 5379840 & 3 & \\
\hline vB 92 & 473.38 & 6.60 & 291.35 & 4.07 & 109.07 & 0.94 & 12.07 & 0.11 & -2.6 & 13.8 & 0.60 & 2.62 & 4096768 & 5380352 & 3 & \\
\hline vB 93 & 330.46 & 2.38 & 204.75 & 2.50 & 76.29 & 0.50 & 8.42 & 0.08 & 7.5 & 8.4 & 0.60 & 2.62 & 4096768 & 5380864 & 3 & \\
\hline vB 96 & 722.91 & 7.20 & 449.19 & 5.48 & 167.82 & 1.11 & 18.54 & 0.17 & -13.6 & 12.3 & 0.60 & 2.62 & 4096768 & 5381376 & 3 & \\
\hline vB 97 & 730.07 & 10.70 & 462.11 & 5.64 & 169.52 & 1.12 & 19.07 & 0.17 & -5.3 & 12.0 & 0.60 & 2.62 & 4096768 & 5381888 & 3 & 2 \\
\hline vB 99 & 314.03 & 2.26 & 197.40 & 2.41 & 73.14 & 0.48 & 8.19 & 0.08 & -4.7 & 12.1 & 0.60 & 2.62 & 4096768 & 5382400 & 3 & \\
\hline vB 106 & 770.31 & 5.55 & 479.30 & 5.85 & 171.24 & 1.13 & 19.42 & 0.17 & 10.3 & 11.5 & 0.02 & 2.62 & 5392640 & 5393152 & & \\
\hline vB 142 & 572.80 & 4.12 & 354.86 & 4.33 & 128.34 & 2.14 & 14.38 & 0.13 & 2.2 & 11.9 & 0.02 & 2.62 & 5329664 & 5330176 & & \\
\hline vB 143 & 626.50 & 4.51 & 391.13 & 4.77 & 139.51 & 0.92 & 15.68 & 0.14 & -4.8 & 11.3 & 0.02 & 2.62 & 5406464 & 5406976 & & \\
\hline vB 176 & 550.88 & 7.35 & 337.21 & 4.11 & 128.40 & 0.95 & 14.26 & 0.13 & -0.6 & 7.2 & 0.60 & 2.62 & 4096512 & 5382912 & 3 & \\
\hline vB 180 & 404.68 & 2.91 & 251.91 & 3.07 & 94.00 & 1.25 & 10.52 & 0.09 & 2.4 & 7.1 & 0.60 & 2.62 & 4096768 & 5383424 & 3 & \\
\hline vB 183 & 266.16 & 1.92 & 168.22 & 2.05 & 63.01 & 0.43 & 7.04 & 0.08 & -11.7 & 12.4 & 0.60 & 2.62 & 4096768 & 5383936 & 3 & \\
\hline
\end{tabular}

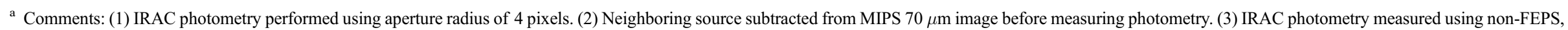
archival data. (4) MIPS photometry measured using non-FEPS, archival data. 
source were observed in all four IRAC bands for the initial verification observations. The IRAC $5.8 \mu \mathrm{m}$ observations were dropped for the remaining sources since it had the lowest $\mathrm{S} / \mathrm{N}$ of the four IRAC bands. The IRAC $5.8 \mu \mathrm{m}$ data are described in Meyer et al. (2004) and are not further discussed here.

Four-band IRAC GTO observations of the 16 Hyades stars and c $2 d$ observations of ScoPMS 214 were observed in full-array, high-dynamic-range mode, where an image is obtained with a $0.6 \mathrm{~s}$ frame time followed by an image with a $12 \mathrm{~s}$ frame time. The Hyades stars were observed at three dither positions, and ScoPMS 214 at two positions in this manner. The $12 \mathrm{~s}$ frame-time images in IRAC 3.6, 4.5, and 5.8 $\mu \mathrm{m}$ bands were saturated and not analyzed.

\subsection{Image Processing}

Analysis was performed on Basic Calibrated Data (BCD) products generated by the data reduction pipeline version $\mathrm{S} 13$ developed at the Spitzer Science Center (SSC). The SSC pipeline removes the electronic bias, subtracts a dark image, applies a flatfield correction, and linearizes the pixel response. Additional processing on the BCD images was performed by the FEPS team as now described.

For the subarray data, cosmic-ray hits were identified and flagged by filtering the sequence of 64 frames at each dither position. At a given pixel, the median and standard deviation of the 64 frames values were computed from the median absolute deviation $^{9}$ to reduce sensitivity to outlier pixel values. Any pixels that deviated from the median by more than $n \sigma$ were flagged, where $n$ was calculated to correspond to a probability of $10^{-4}$ that such an outlier pixel could occur by Gaussian noise given $N$ images (nominally, $N=64$ and $n \approx 4.8$ ). For 26 frames (or $0.03 \%$ of the data), the rejected pixel was within the photometric aperture and the entire frame was discarded. The median and dispersion were recomputed in an iterative fashion until no additional pixels were flagged. For sources HD 77407 and HD 70516, we removed all frames at two dither positions where the FEPS target position overlapped with a latent image.

For the archival full-array observations, cosmic-ray rejection was performed by the MOPEX ${ }^{10}$ (Makovoz \& Marleau 2005) mosaicking package. Images were aligned spatially based on the World Coordinate Systems (WCS) parameters in the image headers. The standard deviation at each pixel position in the stack of aligned images was computed from the median absolute deviation. Pixel values that deviated more than $5 \sigma$ from the median were removed.

After outlier rejection, both the sub- and full-array images were multiplied by the photometric correction images produced by the $\mathrm{SSC}$ that account for variations in the pixel solid angle and the effective response of the filters across the IRAC focal plane. ${ }^{11}$ These correction images were derived by observing a star at 225 positions across the full array and thus link the calibration of sub- and full-array observations.

\subsection{Photometry}

IRAC photometry was measured with a modified version of IDLPHOT. ${ }^{12}$ We measured source flux densities with aperture

\footnotetext{
9 The median absolute deviation (MAD) is defined as MAD $=\operatorname{median}_{i}\left(\mid x_{i}-\right.$ $\operatorname{median}_{j}\left(x_{j}\right) \mid$ ) (Hampel 1974). The standard deviation is estimated from the MAD as $\sigma \approx 1.4826 \mathrm{MAD}$

${ }_{11}$ See http://ssc.spitzer.caltech.edu/postbcd.

11 The correction images are available at http://ssc.spitzer.caltech.edu/irac/ calib.

${ }^{12}$ See http://idlastro.gsfc.nasa.gov.
}

photometry instead of point-response-function (PRF) fitting photometry since the PRF is undersampled in the IRAC $3.6 \mu \mathrm{m}$ band and the PRF shape depends on the position of the star within a pixel. Aperture photometry was performed on each frame with an aperture radius of 3 pixels. This aperture size was chosen as a compromise between $\mathrm{S} / \mathrm{N}$ (which empirically was highest for an aperture radius of 2 pixels) and obtaining accurate calibration between full and subarray observations (which favored larger apertures to reduce the effects of image distortion). The sky background was computed in an annulus on the source centroid with an inner radius of 10 pixels and a width of 10 pixels so that the aperture corrections can be compared directly to values listed in the Spitzer Observing Manual. ${ }^{13}$ Pixel values in the sky annulus were sigmaclipped in an iterative fashion with a clipping threshold of $3 \sigma$, where the dispersion in the sky background was estimated from the median absolute deviation. The sky value was estimated as the mean of the remaining pixels. For several sources, the $\mathrm{S} / \mathrm{N}$ was too low to derive an accurate centroid on individual frames. A subset of frames was then co-added until the formal, internal positional uncertainty was less than 0.1 pixels.

In the IRAC $3.6 \mu \mathrm{m}$ band, the measured flux density can vary up to $3.6 \%$ depending on the distance of the centroid position from the pixel center (Reach et al. 2005), which is defined as the pixel phase $(p)$. This dependency may be caused by nonuniform quantum efficiency across a pixel. The best-fit correction factor $\left(f_{\text {phase }}\right)$ derived from the FEPS data is $f_{\text {phase }}=1.0232-0.0582 p$, which is similar to that obtained by Reach et al. (2005). A correlation of similar magnitude between intensity and pixel phase was found for only one of the four dither positions in the $4.5 \mu \mathrm{m}$ band, and none of the dither positions in the $8 \mu \mathrm{m}$ band. Pixel phase corrections were applied on individual images for the IRAC $3.6 \mu \mathrm{m}$ band only using the above relation.

Aperture corrections are needed to convert the photometry to the fiducial 10 pixel aperture used to calibrate the IRAC instrument (Reach et al. 2005). The multiplicative scaling factor for the 3 pixel aperture was measured from the FEPS data by computing the ratio of the flux density in a 10 pixel aperture to that in a 3 pixel aperture. The derived aperture corrections for a 3 pixel wide aperture are 1.109, 1.110, and 1.200 for IRAC bands 3.6, 4.5 , and $8 \mu \mathrm{m}$, respectively. These aperture corrections agree with the values listed on the SSC IRAC Data Handbook ${ }^{14}$ to within $0.3 \%$ for the 3.6 and $4.5 \mu \mathrm{m}$ bands, and $1.5 \%$ for the $8 \mu \mathrm{m}$ band. The measured aperture corrections for 23 sources deviated by more than $3 \sigma$ from the nominal value. Twenty of these sources are known from an adaptive optics survey to be multiple systems with a separation of $\lesssim 2^{\prime \prime}$ between the primary and secondary components (S. Metchev 2007, private communication). The other three sources have not been observed at high resolution and the multiplicity status is unknown. For these 23 stars, the measured aperture correction at a four pixel radius is within $1.3 \%$ of the nominal correction for each source, and a four pixel aperture radius was used with aperture corrections of 1.069, 1.079, and 1.081 for IRAC bands 3.6, 4.5, and $8 \mu \mathrm{m}$, respectively. These stars are noted in Table 1.

Flux densities were computed as the unweighted average of the flux densities measured in $N$ dither positions ( $N=4$ nominally). The standard deviation of the $N$ dither positions ( $\left.\equiv \sigma_{\mathrm{rms}}\right)$, normalized by the mean flux density, is plotted versus the mean flux density in Figure 1. For the IRAC 3.6 and $4.5 \mu \mathrm{m}$ bands, the normalized rms shows no trend with mean flux density, while for IRAC $8 \mu \mathrm{m}$, the normalized rms increases systematically toward

\footnotetext{
13 See http://ssc.spitzer.caltech.edu/documents/SOM.
}

14 See http://ssc.spitzer.caltech.edu/irac/dh. 


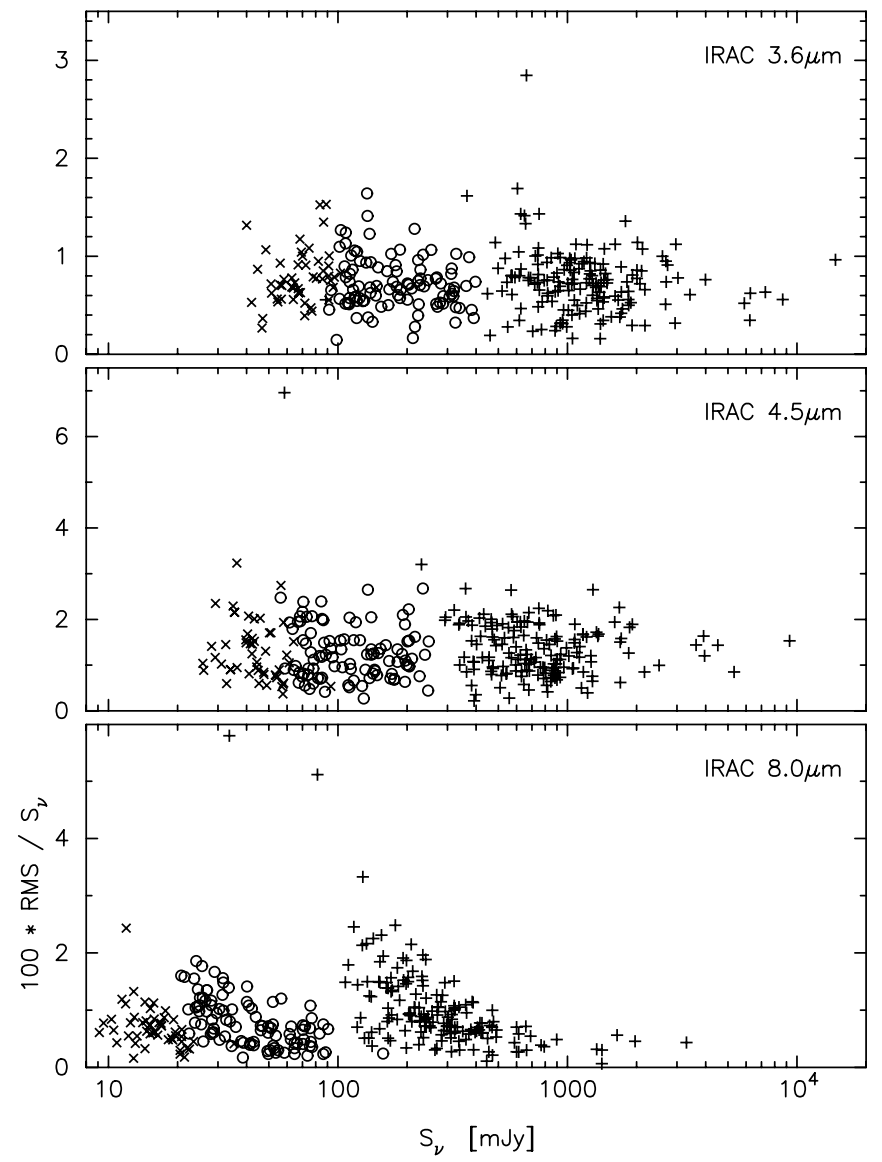

FIG. 1.- Normalized rms of the measured flux densities in the four subarray dither positions plotted vs. the mean flux density for the FEPS IRAC subarray observations. Stars observed with IRAC frame times of $0.02,0.1$, and $0.4 \mathrm{~s}$ are represented by plus signs, open circles, and times signs, respectively. We used the repeatability between the dithered observations to assign a minimum photometric uncertainty of $0.72 \%, 1.22 \%$, and $0.66 \%$ for IRAC bands $3.6,4.5$, and $8 \mu \mathrm{m}$, respectively.

fainter sources for a fixed frame time. This trend is expected if the $\mathrm{S} / \mathrm{N}$ is photon limited and the integration time is constant since fainter sources will have lower $\mathrm{S} / \mathrm{N}$. The photometric repeatability at a fixed dither position indicates that the standard deviation of the photometry computed from the four dither positions should be $<0.4 \%$ in each band for the brighter stars. Given that the repeatability between dithers is poorer, the photometric precision is limited by either our data reduction procedures or instrumental limitations in obtaining dithered data. Internal photometric uncertainties were therefore computed as $\sigma_{\text {rms }} / \sqrt{N}$, but with a minimum uncertainty imposed. For the IRAC 3.6 and $4.5 \mu \mathrm{m}$ bands, we adopt a minimum internal uncertainty of $0.72 \%$ and $1.22 \%$, respectively, which corresponds to the median repeatability from the ensemble data shown in Figure 1. For the IRAC $8 \mu \mathrm{m}$ band, we adopt a minimum uncertainty of $0.66 \%$, which is the median value for stars with a repeatability less than $1.2 \%$.

We investigated the relative calibration of IRAC sub- and fullarray data since the flux calibrators used by Reach et al. (2005) were observed in full-array mode. To compare the subarray and full-array calibration, we analyzed observations of the star HD 135285 that were obtained by the SSC in full-array mode and in subarray mode with $0.4 \mathrm{~s}$ integration times. The mean ratio of the flux densities measured in subarray mode to that in full-array mode is $1.004 \pm 0.004$ for the IRAC $3.6 \mu \mathrm{m}$ band, $1.001 \pm$ 0.004 for $4.5 \mu \mathrm{m}, 0.995 \pm 0.002$ for $5.8 \mu \mathrm{m}$, and $0.997 \pm 0.001$ for $8 \mu \mathrm{m}$. The weighted mean for all four bands is $0.997 \pm 0.001$.
We conclude that any calibration offsets between the $0.4 \mathrm{~s}$ subarray mode and full-array mode is less than $1 \%$, and no further calibration corrections were applied to the subarray observations. In $\S 7$ we consider the relative subarray calibration for the different frame times.

The IRAC photometry and internal uncertainties are presented in Table 1 . The flux density measurements are tied to the calibration described in Reach et al. (2005) with calibration factors of $0.0188,0.1388$, and $0.2021 \mathrm{MJy} \mathrm{sr}^{-1}$ per DN s${ }^{-1}$ for IRAC 3.6, 4.5 , and $8 \mu \mathrm{m}$, respectively, and a $1 \sigma$ uncertainty of $2 \%$.

\section{MIPS $24 \mu \mathrm{m}$}

The MIPS instrument obtains images in the 24,70 , and $160 \mu \mathrm{m}$ bands. This section describes the data reduction procedures for the $24 \mu \mathrm{m}$ band. The $70 \mu \mathrm{m}$ data are discussed in $\S 4$, and analysis of the $160 \mu \mathrm{m}$ data are presented in Kim et al. (in preparation). The $128 \times 128$ pixel MIPS $24 \mu \mathrm{m}$ array images an instantaneous field of view of $\sim 5.4^{\prime} \times 5.4^{\prime}$ region with a pixel scale of $2.5^{\prime \prime} \times 2.6^{\prime \prime}$. The FEPS team obtained MIPS $24 \mu \mathrm{m}$ observations in photometry mode for 323 sources. Data for five stars (HD 17925, HD 72905, HD 202917, HD 216803, ScoPMS 214) were extracted from the Spitzer archive. The exposure time (either 3 or $10 \mathrm{~s}$ ) and the number of dithered images (either 28 or 56) were set to achieve a signal to noise of at least 30 on the expected stellar photosphere brightness.

\subsection{Image Processing}

MIPS $24 \mu \mathrm{m}$ images for all but one source were processed with SSC pipeline version S13. The star HD 143006 has a flux density of $\sim 3 \mathrm{Jy}$ at $24 \mu \mathrm{m}$, and S13 data products have an error in the linearity correction for such bright sources. For HD 143006 only, we used S16 data products where the linearity problem was fixed.

Individual BCD images that contain the "strong" jailbar effect caused by bright sources or cosmic rays were removed on visual inspection. Images were also removed if cosmic-ray hits were found near the expected source position. These images were identified by performing aperture photometry on individual BCD images, and finding outlier flux densities or centroid coordinates compared to the mean that had less than a $10^{-4}$ chance to have been caused by random noise.

Once contaminated BCD images were removed, additional processing steps were performed following the recommendations from the SSC MIPS handbook and the MIPS instrument team (Engelbracht et al. 2007). First, for a given source, background levels in individual images were adjusted to a common median value using an additive constant. Images for a given source were then median combined to derive a flat-field image which removes long-term gain changes in the MIPS array. For the median filtering, a 5 pixel radius region centered on the source position was masked. A $3 \sigma$ clipping algorithm was used to remove outliers on a pixel-by-pixel basis through the image stack. The resulting median image was normalized by the median pixel value over the image. Flat-field images were derived only for sources that are not surrounded by nebulosity. Affected sources were identified from visual inspection of the image mosaics. If nebulosity is present, a flat-field image from another FEPS source was used that was (1) obtained within a time interval \pm 1 day, (2) had the same exposure time, and (3) had the closest matched background level. If no such image existed, the image nearest in time with the same exposure time was used. The stability of the flats over time were assessed by taking the ratio of flats taken on different days. Over a \pm 3 day period, the mean flat-field value 
for the central $5 \times 5$ pixel region of the MIPS $24 \mu \mathrm{m}$ array is repeatable to $1.4 \%$ peak-to-peak with a dispersion of $0.2 \%$.

\subsection{Photometry}

Photometry was performed with the MOPEX package (Makovoz \& Marleau 2005). The BCD images for a given source were aligned spatially based on the WCS information in the image headers. Cosmic-ray rejection was performed by removing pixels within the stack that deviated by more than $5 \sigma$ from the mean. Point sources were identified on a mosaic of the BCD images using a $10 \sigma$ detection threshold. The detection list was modified after visual inspection of the mosaics to remove spurious sources and to add any sources missed by the automated detection method.

PRF fitting photometry was performed with the APEX module in MOPEX. PRF fitting photometry was chosen over aperture photometry since the PRF is critically sampled in the MIPS $24 \mu \mathrm{m}$ images and should provide the optimum signal to noise. The empirical PRF distributed with the APEX package was fitted to the individual BCD images simultaneously (as opposed to the mosaicked image) using a fitting area of $21 \times 21$ pixels for most images. A $5 \times 5$ pixel fitting area was used for 11 sources that have spatially variable nebulosity near the point source position. From visual inspection of the mosaicked images, the PRF from other $24 \mu \mathrm{m}$ sources sometimes overlapped with the PRF from the FEPS target. These contaminating sources were fitted with a PRF simultaneously with the FEPS target. The free parameters in the PRF fit include a spatially constant sky background level, and the peak flux density and centroid position for each source.

Photometry was measured by integrating the fitted PRF within a 3 pixel radius $\left(1\right.$ pixel $\left.\sim 2.55^{\prime \prime}\right)$ since the wings of the PRF have lower signal to noise. An aperture correction is then needed to place the PRF photometry on the zero-point scale adopted by the MIPS instrument team. The aperture correction was derived by measuring aperture photometry on individual BCD images using a customized version of IDLPHOT. We adopted an aperture radius of $13^{\prime \prime}$ and a sky annulus between $20^{\prime \prime}$ and $32^{\prime \prime}$ since these aperture parameters have been calibrated by the MIPS instrument team to a theoretical PRF. Aperture flux densities were computed as the unweighted mean of the photometry measured on individual BCD images. The average ratio of the flux density measured with $13^{\prime \prime}$ aperture photometry compared to 3 pixel $\left(7.65^{\prime \prime}\right)$ PRF photometry is 1.371 with a dispersion of 0.011 for 108 sources brighter than $20 \mathrm{mJy}$. From the SSC World Wide Web site, ${ }^{15}$ the aperture correction for a $13^{\prime \prime}$ aperture radius and the adopted sky annulus is a 1.167 . The final flux densities were obtain by multiplying the PRF flux densities by the product of these factors (1.600).

Internal uncertainties computed by APEX are often much smaller $(\ll 1 \%)$ than is assessed from repeated observations of the source. The minimum internal uncertainty was estimated based on photometric repeatability from aperture photometry. The normalized rms of the MIPS $24 \mu \mathrm{m}$ flux densities measured from aperture photometry on individual BCD images is presented in Figure 2. For sources brighter than $100 \mathrm{mJy}$, the mean rms repeatability is $0.9 \%$ in a 3 pixel aperture radius, which we adopted as the minimum uncertainty for the PRF photometric uncertainties.

The MIPS $24 \mu \mathrm{m}$ photometry and internal uncertainties are presented in Table 1 . The S13 images were processed with a calibration factor of $0.0447 \mathrm{MJy} \mathrm{sr}^{-1}$. Following Engelbracht et al. (2007), we adopt a calibration uncertainty of $4 \%$.

\footnotetext{
15 See http://ssc.spitzer.caltech.edu/mips/apercorr.
}

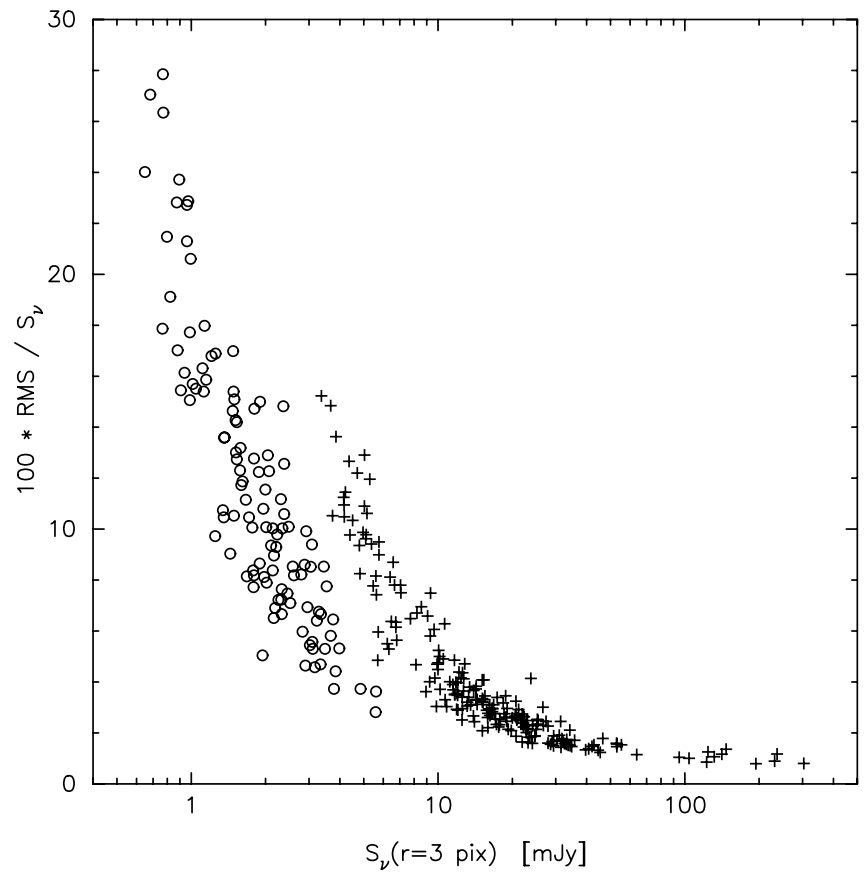

FIG. 2.- Repeatability of the rms of the MIPS $24 \mu \mathrm{m}$ aperture photometry measured in a 3 pixel radius on individual BCD images. Crosses represent sources observed with an exposure time of $3 \mathrm{~s}$, and open circles with $10 \mathrm{~s}$. We adopted a minimum uncertainty of $0.9 \%$ based on the mean repeatability for stars brighter than $100 \mathrm{mJy}$.

\section{MIPS $70 \mu \mathrm{m}$}

We obtained MIPS $70 \mu \mathrm{m}$ observations for 323 sources and extracted data for five stars (HD 17925, HD 72905, HD 202917, HD 216803, ScoPMS 214) from the Spitzer archive. The FEPS observations were obtained in photometry mode with an exposure time of $10 \mathrm{~s}$ and the small field size dither pattern. A single MIPS $70 \mu \mathrm{m}$ image in this mode contains $32 \times 32$ pixels with a scale of $9.8^{\prime \prime}$ pixel $^{-1}$. The FEPS sources were centered on the left half of the array which had the best sensitivity. The number of cycles per source ranged between 2 and 10, where a cycle contains up to 12 dithered images. The number of cycles were set based on the stellar distance and age to reach the expected brightness of the outer solar system dust level at that stellar age (see Hillenbrand et al. 2008).

\subsection{Image Processing}

MIPS $70 \mu \mathrm{m}$ images were processed with SSC pipeline version S13 that removes the bias, subtracts a dark image, applies a flat-field correction, and linearizes the pixel response. Individual BCD images were mosaicked with the Germanium Reprocessing Tools (GeRT) software package S14.0 version 1.1 developed at the SSC. The GeRT package performs column spatial filtering on the BCD images and then a time median filter to remove residual pixel response variations. A $40^{\prime \prime} \times 40^{\prime \prime}$ region centered on the source position, compared to the PRF FWHM size of $18^{\prime \prime}$, was excluded when computing the column and time filtering such that the filtering process is not biased by the presence of a bright source. Filtered images were formed into mosaics with MOPEX (Makovoz \& Marleau 2005). Outlier pixels were rejected using a $3 \sigma$ clipping threshold.

\subsection{Photometry}

Aperture photometry was performed on the MIPS $70 \mu \mathrm{m}$ mosaics with a custom version of IDLPHOT. We adopted aperture 
photometry over PRF fitting photometry since most sources were not detected at $70 \mu \mathrm{m}$, and aperture photometry enables a straightforward interpretation of the upper limits.

The adopted aperture radius of $16^{\prime \prime}$ (4 pixels on the co-added images), which corresponds to approximately the FWHM size of the PRF, was chosen to optimize the $\mathrm{S} / \mathrm{N}$ for faint sources (see, e.g., Naylor 1998). The sky level was computed as the mean pixel value in a sky annulus that extends from $40^{\prime \prime}$ to $60^{\prime \prime}$ after performing the iterative clipping procedure described in $\S 2.2$. The aperture was centered on the expected stellar position computed from the WCS parameters contained in the FITS image headers, and no centroiding was performed. Visual inspection of the $70 \mu \mathrm{m}$ mosaics identified 19 images where a point source was located within the outer sky annulus or the aperture radius, but offset from the stellar position determined from 2MASS astrometry. A PRF was fitted to the contaminating source and subtracted from the image using MOPEX. These 19 sources are identified in Table 1. Aperture photometry was recomputed on the PRF-subtracted image.

The $70 \mu \mathrm{m}$ photometric uncertainty was computed as

$$
\sigma=\left(\eta_{\text {sky }} \eta_{\text {corr }}\right)\left(\Omega \Sigma_{\text {sky }}\right) \sqrt{N_{\text {ap }}+N_{\text {ap }}^{2} / N_{\text {sky }}},
$$

where $\Sigma_{\text {sky }}$ is the noise per pixel in units of surface brightness as measured in the sky annulus, $\Omega$ is the solid angle of a pixel, $N_{\text {ap }}$ is the number of pixels in the aperture, $N_{\text {sky }}$ is the number of pixels in the sky annulus, and $\eta_{\text {sky }}$ and $\eta_{\text {corr }}$ correct for correlated noise terms as described below. The total uncertainty is the rms sum of two terms: the term proportional to $\left(N_{\mathrm{ap}}\right)^{1 / 2}$ is the uncertainty from random fluctuations in the pixel noise summed over the aperture, and the term proportional to $\left(N_{\mathrm{ap}}^{2} / N_{\text {sky }}\right)^{1 / 2}$ represents the uncertainty in the mean pixel noise from the sky annulus (often assumed to be zero due to the large area over which one usually measures the mean sky).

Two correction factors are needed to compute accurate uncertainties. Because the $70 \mu \mathrm{m}$ mosaics were sampled at a finer scale than the raw images, the noise between adjacent pixels is correlated. The factor $\eta_{\text {corr }}$ accounts for the correlated noise, and was estimated as the ratio of the pixel size in the raw images $\left(9.8^{\prime \prime}\right)$ to that in the mosaics $\left(4^{\prime \prime}\right)$, or $\eta_{\text {corr }}=2.5$.

The second correction factor, $\eta_{\text {sky }}$, accounts for systematic differences in the pixel noise between the aperture and sky annulus. Variations in the pixel noise as a function of position across the mosaics were assessed by first scaling all $70 \mu \mathrm{m}$ mosaics in the FEPS program to a common median value. The standard deviation of each pixel in the stack of mosaic images was computed after removing 35 images where the FEPS target was clearly detected. The resulting image showed that the mosaic noise was higher along the columns near the source position due to timevariable latent images from the calibration stim flashes. The pixel noise was estimated to be $40 \%$ higher in the aperture compared to the sky annulus, and we adopt $\eta_{\text {sky }}=1.40$.

Figure 3 shows a histogram of the $\mathrm{S} / \mathrm{N}$ for the $70 \mu \mathrm{m}$ photometry. Visual inspection of the mosaicked images indicates that the majority of the FEPS sources have not been detected at $70 \mu \mathrm{m}$. The histogram in Figure 3 should then be a Gaussian with unit dispersion (Fig. 3, solid curve) if equation (1) contains the dominant noise terms. In practice, the observed $\mathrm{S} / \mathrm{N}$ distribution is broader than the expected Gaussian distribution and includes $\mathrm{S} / \mathrm{N}$ values as low as -4.2 .

As shown by the dotted curve in Figure 3, a Gaussian with a dispersion of 1.49 adequately describes the observed distribution. The origin of the apparent excess noise is unknown, but none-

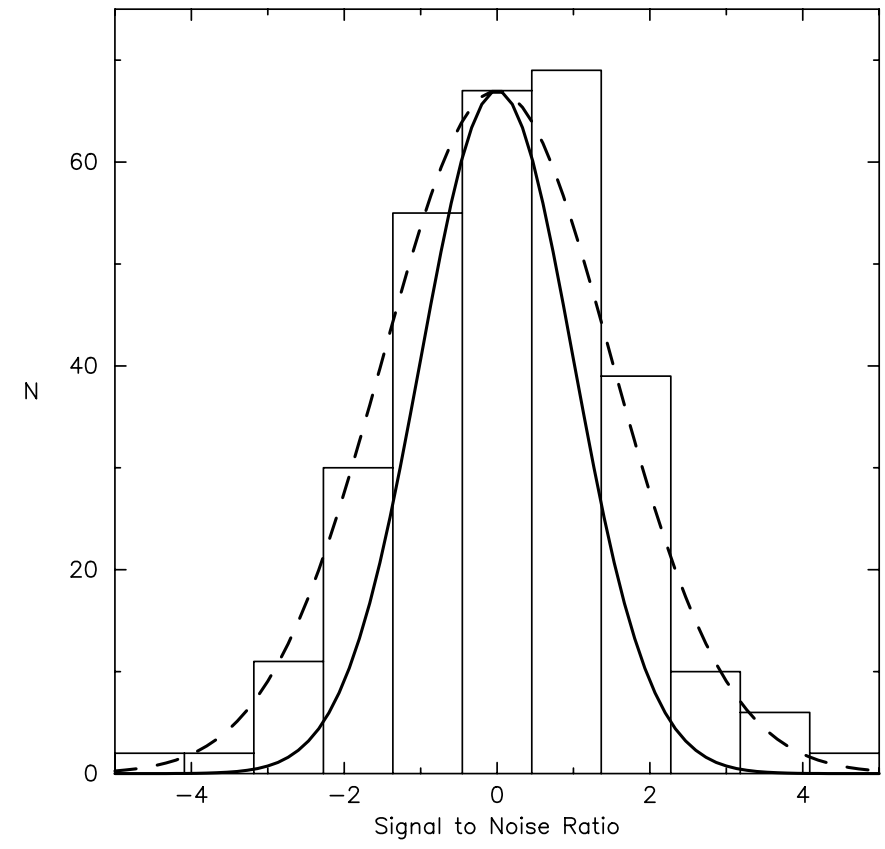

FIG. 3.- Histogram of the $\mathrm{S} / \mathrm{N}$ measured in a $16^{\prime \prime}$ radius aperture at the expected stellar position in the MIPS $70 \mu \mathrm{m}$ mosaics. The solid curve shows the expected $\mathrm{S} / \mathrm{N}$ distribution for Gaussian noise (dispersion $=1.0$ ) scaled to a peak value of $\mathrm{N}=67$. The dashed curve shows a Gaussian with a dispersion of 1.49 . These results suggest that the $70 \mu \mathrm{m}$ photometric uncertainties are underestimated by a factor of 1.49 . The uncertainties in the $70 \mu \mathrm{m}$ flux densities reported in Table 1 have been scaled by this factor.

theless, we have scaled the photometric uncertainties for all sources by a factor of 1.49 .

MIPS $70 \mu \mathrm{m}$ photometry is calibrated to a theoretical PRF measured computed over a $64^{\prime} \times 64^{\prime}$ field (Gordon et al. 2007). The aperture correction needed to place the background-subtracted flux densities measured in a finite aperture on the same scale as the theoretical PRF depends on the temperature of the underlying source emission. In anticipation that the FEPS MIPS $70 \mu \mathrm{m}$ observations did not detect the stellar photosphere in most cases and that debris disks around solar-type stars have temperatures of $\sim 50-100 \mathrm{~K}$, aperture corrections were measured on a $100 \mathrm{~K}$ PRF (Gordon et al. 2007). The aperture correction derived for our adopted aperture radius of $16^{\prime \prime}$ and sky annulus between $40^{\prime \prime}$ and $60^{\prime \prime}$ is 1.766 . By comparison, the SSC web pages indicate that the aperture of correction for a 3000 and $15 \mathrm{~K}$ PRF is 1.741 and 1.884 , respectively, for the same $16^{\prime \prime}$ aperture radius and similar, but not identical, background annulus of $39^{\prime \prime}-65^{\prime \prime}$.

The MIPS $70 \mu \mathrm{m}$ photometry and internal uncertainties are presented in Table 1. The FEPS sources where the $70 \mu \mathrm{m}$ photometry was measured on PRF-subtracted images are marked in the table. The adopted calibration factor is $702.0 \mathrm{MJy} \mathrm{sr}^{-1} /\left(\mathrm{DN} \mathrm{s}^{-1}\right)$ with an uncertainty of $7 \%$ as reported on the SSC MIPS calibration web pages. ${ }^{16}$

\section{IRS LOW-RESOLUTION SPECTRA}

Low-resolution spectra $(\lambda / \delta \lambda \sim 60-120)$ of the FEPS sources were obtained with IRS. Most sources were observed in the shortlow 1 (SL1, 7.4-14.5 $\mu \mathrm{m}$ ), long-low 2 (LL2, 14.0-21.3 $\mu \mathrm{m}$ ), and long-low 1 (LL1, 19.5-38 $\mu \mathrm{m}$ ) orders. A subset of sources were also observed in the short-low 2 (SL2, 5.2-7.7 $\mu \mathrm{m}$ ) order. The spectral coverage beyond $35 \mu \mathrm{m}$ suffers from low $\mathrm{S} / \mathrm{N}$ and was discarded for all sources. The source HD 191089 was observed

16 See http://ssc.spitzer.caltech.edu/mips/calib. 
by a GTO program (PID 2; PI: J. Houck) and was not included in the FEPS IRS observations. Also, HD 72905 and HD 216803 were observed in the SL2 order only by FEPS; the longer wavelengths for HD 72905 were observed as part of a GTO program (PID 41; PI: G. Rieke).

Targets were acquired in the spectrograph slit using either highaccuracy IRS or PCRS peak-up with a $1 \sigma$ radial pointing uncertainty of $0.4^{\prime \prime}$ and $0.14^{\prime \prime}$, respectively, according to version 8.0 of the Spitzer Observing Manual. The reconstructed pointing from the peakup observations differed from the requesting pointing by $>9$ " for five sources: HD 80606, HD 139813, HII 2881, HIP 42491, and RX J1544.0-3311. We assumed that the spectra for these five sources are not for the intended target. For HD 13974 , the pointing offset was within the pointing accuracy of the IRS peakup, but the intensity of the SL1 spectrum is a 2.6 times lower than expected by extrapolating the IRAC $8 \mu \mathrm{m}$ photometry to $13 \mu \mathrm{m}$ assuming a $\nu^{2}$ spectrum. For R45, the extracted spectrum had a flux density less than 0 Jy for wavelengths $>25 \mu \mathrm{m}$. We have excluded the SL and LL spectra for HD 13974 and R45, respectively.

Two nod positions per cycle were obtained for the IRS observations in standard staring mode with a minimum of six cycles per target to allow rejection of bad pixels and cosmic-ray hits. Each spectral image comes with a bit-mask image that marks potentially bad pixels. The data conditions identified by each bit in the mask are described in the Spitzer Data Handbook. ${ }^{17}$ Pixels marked with bit 9 or higher were replaced with the average pixel value of an 8 pixel box surrounding the bad pixel. This method for finding the mean pixel value resembles Nagao-Matsuyama filtering (Nagao \& Matsuyama 1979) and ensured edge preservation in the source region of our spectral images.

Source spectra were extracted from the droop intermediate data product from the SSC pipeline version S13 for all but two sources. The spectra for MML 18 and ScoPMS 52 were reobserved since the initial observations had a failed peakup, and the final spectra were extracted from S15 data products. Background emission and stray light were eliminated by subtracting images of the two slit positions at which a target is observed for each module and order. This resulted in a set of images containing a positive and negative spectrum in each observed order. A straight-sided (boxcar) aperture was used to extract the spectra for each nod position and cycle.

We found that the source positioning has the expected $0.4^{\prime \prime}(1 \sigma)$ pointing accuracy, but that the targets are not positioned exactly on the $\frac{1}{3}-\frac{2}{3}$ position along the slit. The width of each aperture was determined by two quantities: the maximum size of the PSF in each order, and the pointing accuracy. The width of each aperture is chosen such that $99 \%$ of the source flux is within the aperture. To estimate the size of a point source we assume a Gaussian PSF with a FWHM $=0.25^{*} \lambda$, where $\lambda$ is in microns and FWHM is in arcseconds. Taking also the positioning constraints into account, the apertures are widened an additional 2.4" $(6 \sigma)$, to ensure that the entire source is always positioned within the aperture. The resulting extraction boxes were 6 pixels $\left(11.1^{\prime \prime}\right)$ and 5 pixels $\left(25.4^{\prime \prime}\right)$ along the slit for the short-low and long-low modules, respectively. Given that the slit width is only 2 pixels, pointing uncertainties in the dispersion direction will dominate the error on the flux density.

Because spectra were extracted with custom apertures that differ from the SSC processing, the spectral response function (SRF) had to be derived. We used a set of high $\mathrm{S} / \mathrm{N}$ observations of bright calibration stars with model spectra provided by the SSC to derive

17 See http://ssc.spitzer.caltech.edu/irs/dh. the relative SRF, and then an internal calibration to determine the absolute flux calibration. Calibrating slit spectra suffers from uncertainties in the adopted spectral model and flux losses due to pointing offsets of the slit compared to the target. The FEPS Legacy program provides a unique opportunity to derive a good flux calibration for solar-type main-sequence stars since many stars do not exhibit emission from cool dust in the IRS wavelengths (Carpenter et al. 2008). The SRFs were determined for each order separately as the ratio of the observed spectrum to a Kurucz model spectrum using calibration stars identified in the FEPS program. The Kurucz model spectra were derived using the procedure outlined in Appendix C. Calibration stars were selected from the FEPS program by computing synthetic fluxes from the IRS spectra at wavelengths of $8,13,24$, and $33 \mu \mathrm{m}$ and applying the following criteria: (1) the flux density ratios of the synthetic photometry points at $8,13,24$, and $33 \mu \mathrm{m}$ are within $1 \sigma$ of the colors expected for stellar photospheres; (2) there were no known peak-up problems during data acquisition; (3) the spectra contains no artifacts from cosmic-ray hits, hot or dead pixels; and (4) the spectra have among the best $\mathrm{S} / \mathrm{N}$ for the specific order and ramp time to ensure high-quality SRFs. The SRFs were derived from a set of 16 calibration stars for the SL1 and LL orders, and from a separate set of 10 stars for the SL2 order.

After the extraction of each spectrum and normalization by the SRF, a mean spectrum over all slit positions and cycles was computed for each individual order. The orders were then combined to form a single spectrum. In the regions where the spectra of the individual orders overlap, the flux densities were replaced by the mean flux density at each wavelength point. Internal uncertainties per pixel were estimated as the standard deviation of the mean of the repeated spectral observations. The SRF based on the bright calibration stars from the IRS instrument team (the spectra were extracted in an identical way to the FEPS sample) were then scaled to the SRF derived from the internal calibration described above. This procedure ensures that the uncertainties introduced by the adopted spectral model and flux losses due to pointing offsets of the slit are minimized and that the signal-to-noise ratio on the relative SRF is much better than that of the spectrum of any individual target.

The final calibrated spectra, excluding the problem spectra mentioned above, are distributed in the electronic version of this article. Each data file contains a header summarizing the observational parameters and four data columns that list the wavelength in microns, the flux density and internal uncertainty in Janskys, and the spectral order number.

\section{SOURCE CONFUSION}

Infrared cirrus and extragalactic sources may contaminate the FEPS photometry and create the appearance of an infrared excess. Since we anticipate that the emission associated with the stellar photosphere or a circumstellar disk will be nearly pointlike and centered on the star at the typical distances in the FEPS sample, potential contamination to the 24 or $70 \mu \mathrm{m}$ photometry can be identified from emission that is extended or offset from the stellar position.

We used the 2MASS catalog to represent the stellar position since most stars in the FEPS sample do not exhibit an infrared excess in the $J H K_{s}$ bands (Carpenter et al. 2008), and any such excess should be unresolved spatially. 2MASS astrometry was corrected to the epoch of the Spitzer observations based on proper motions in the Tycho-2 (Høg et al. 2000) or UCAC2 (Zacharias et al. 2004) astrometric catalogs. MIPS $24 \mu \mathrm{m}$ source coordinates were computed from the PRF centroid position and the WCS astrometric solution in the FITS image headers. 


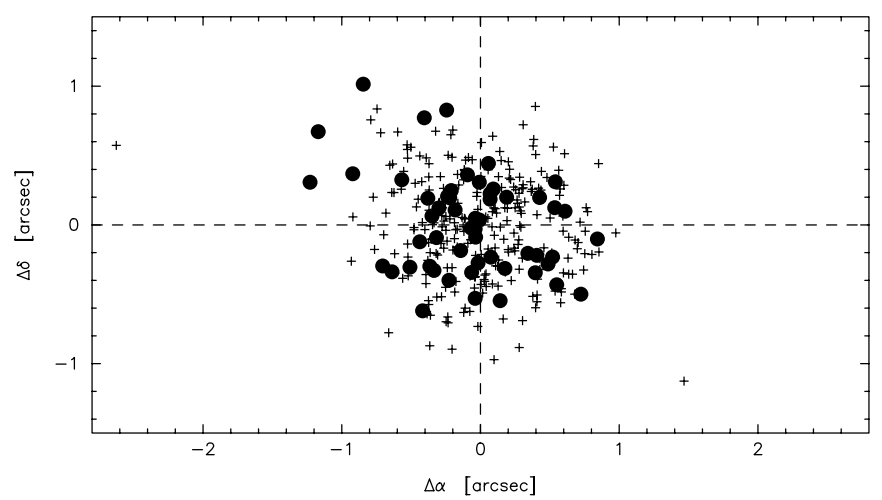

FIG. 4.- Angular offset between the $24 \mu \mathrm{m}$ emission centroid and the $2 \mathrm{MASS}$ position after correcting for proper motion and differences in epoch of observations. Filled circles represent FEPS stars that exhibit an infrared excess in the IRS spectrum, and crosses represent sources without a detectable IRS excess (Carpenter et al. 2008).

In Figure 4 we show the angular separation between the 2MASS and MIPS $24 \mu \mathrm{m}$ astrometry, where solid circles represent sources that exhibit an infrared excesses in the IRS spectra and crosses indicate stars without an excess (see Carpenter et al. 2008). Two sources have $24 \mu \mathrm{m}$ positions that are offset by more than $1.8^{\prime \prime}$ from the 2MASS coordinates, but neither exhibits an IRS infrared excess. Excluding these two outliers, the dispersion in the right ascension and declination offsets are $0.41^{\prime \prime}$ and $0.36^{\prime \prime}$, respectively, with a radial dispersion of $0.49^{\prime \prime}$. The dispersion is dominated by uncertainties in the Spitzer astrometry since the typical $1 \sigma$ uncertainty in the 2MASS positions is $\sim 0.14^{\prime \prime}$ (Skrutskie et al. 2006). Stars with infrared excesses have a larger dispersion in the radial coordinate offsets than stars without infrared excesses $\left(0.30^{\prime \prime}\right.$ vs. $\left.0.23^{\prime \prime}\right)$, which can be attributed to three excess sources (HD 35850, HD 201219, and HD 209253) that have offsets of $\sim 1.3^{\prime \prime}$. The $24 \mu \mathrm{m}$ excess source with the largest angular offset, which is HD 35850 at $1.35^{\prime \prime}$, deviates from the 2MASS position by $2.9 \sigma$ in right ascension and $1.9 \sigma$ in declination. We conclude that for most FEPS sources, the $24 \mu \mathrm{m}$ astrometry offsets relative to $2 \mathrm{MASS}$ is similar for stars with and without an infrared excess. Potentially three excesses sources may be contaminated by cirrus or extragalactic sources to produce an unusually large offset $\left(1.3^{\prime \prime}\right)$. However, we cannot rule out pointing reconstruction errors since the two largest astrometric offsets are found around stars without infrared excesses.

The relative MIPS $70 \mu \mathrm{m}$ and 2MASS astrometry was evaluated in a similar manner. We computed the $70 \mu \mathrm{m}$ emission centroid by fitting a two-dimensional Gaussian to a $44^{\prime \prime} \times 44^{\prime \prime}$ $(11 \times 11$ mosaicked pixels $)$ region centered on the expected stellar position. In Figure 5 we show the difference between the $70 \mu \mathrm{m}$ and 2MASS astrometry as a function of the $70 \mu \mathrm{m} \mathrm{S} / \mathrm{N}$ measured in a $16^{\prime \prime}$ aperture. For sources with $\mathrm{S} / \mathrm{N} \geq 3$, the positional agreement is better than $3.5^{\prime \prime}$ for all but three sources: HD 201219 (5.1" offset), HD 104467 (12.8"), and RX J1111.7-7620 (13.4"). RX J1111.7-7620 is separated by $24.4^{\prime \prime}$ from the classical T Tauri star XX Cha; these sources have comparable brightness at $70 \mu \mathrm{m}$ and the Gaussian fit converged to a centroid intermediate between the two sources. The $70 \mu \mathrm{m}$ detection toward HD 104467 is a point source clearly offset from the stellar position. Given the large offset, we assume that the detected $70 \mu \mathrm{m}$ source is unrelated to the star. Finally, the HD $20121970 \mu \mathrm{m}$ mosaic contains two point sources separated by $20.9^{\prime \prime}$ that distorted the Gaussian fit. We fitted Gaussians to both sources and determined that the brighter of the two sources is $3.4^{\prime \prime}$ from the 2MASS position for HD 201219, which is not unusual given the $70 \mu \mathrm{m} \mathrm{S} / \mathrm{N}$ (5.9) for this source.

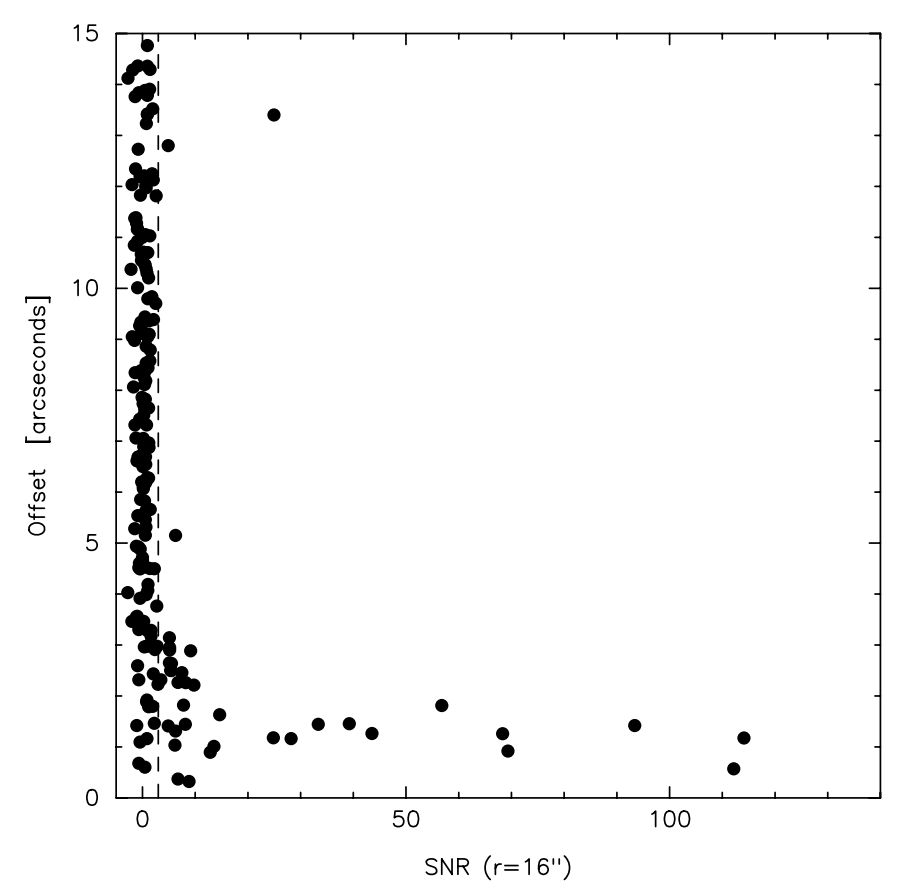

FIG. 5.-Angular offset between the $70 \mu \mathrm{m}$ coordinates and the 2MASS stellar position as a function of the $70 \mu \mathrm{m} \mathrm{S} / \mathrm{N}$ measured in a $16^{\prime \prime}$ radius aperture. The $70 \mu \mathrm{m}$ centroid was computed by fitting a two-dimensional Gaussian to a $44^{\prime \prime} \times$ $44^{\prime \prime}$ region centered on the stellar position. The vertical dashed line at $\mathrm{S} / \mathrm{N}=3$ indicates the minimum signal-to-noise ratio that defines a MIPS $70 \mu \mathrm{m}$ detection. 2MASS coordinates have been corrected to the Spitzer epoch of observations using published proper motions.

However, this source also exhibits one of the larger angular offsets between the MIPS $24 \mu \mathrm{m}$ astrometry and 2MASS. While neither the $24 \mu \mathrm{m}$ nor the $70 \mu \mathrm{m}$ astrometry conclusively demonstrates that the MIPS photometry for HD 201219 is contaminated, it suggests that the photometry for this source should be used with caution.

To further search for possible contaminants in the MIPS photometry, we computed the ratio of the flux measured in a large (10.2" and $30^{\prime \prime}$ for MIPS 24 and $70 \mu \mathrm{m}$, respectively) to a small (5.2" and $16^{\prime \prime}$ for MIPS 24 and $70 \mu \mathrm{m}$ ) aperture radius. A contaminating object or extended emission will create an anomalous ratio between aperture sizes. In Figure 6 we show the flux ratio measured in a large aperture to that in a small aperture as a function of the $\mathrm{S} / \mathrm{N}$ for the MIPS $24 \mu \mathrm{m}$ photometry. The scatter in the flux ratio is similar for sources with (solid circles) and without (crosses) $24 \mu \mathrm{m}$ excesses. For $\mathrm{S} / \mathrm{N}>300$, the source with the most discrepant flux ratio at $24 \mu \mathrm{m}$ relative to the other sources is HD 107146 at $\mathrm{S} / \mathrm{N}=900$. Several studies have demonstrated that this source is surrounded by a circumstellar disk (Ardila et al. 2004; Williams et al. 2004; Carpenter et al. 2005) and the observed flux ratio suggests that the source may be extended at $24 \mu \mathrm{m}$. Sources with a $24 \mu \mathrm{m} \mathrm{S} / \mathrm{N}$ ratio less than 100 exhibit a larger scatter in flux ratios. The range of values is similar for sources with and without infrared excesses, and suggests that the scatter can be attributed to lower signal-to-noise in the larger photometric aperture.

In Figure 7 we show the flux ratio in the two aperture sizes as a function of the $\mathrm{S} / \mathrm{N}$ for the MIPS $70 \mu \mathrm{m}$ photometry. Two sources (HD 104467 and RX J1111.7-7620) with S/N > 3 have anomalously large ratios $(>1.8)$. As discussed above, the initial photometry for these sources were contaminated by a nearby object, and the nearby source was PRF-subtracted before performing the final photometry. A third source (HD 216803) has a flux ratio 


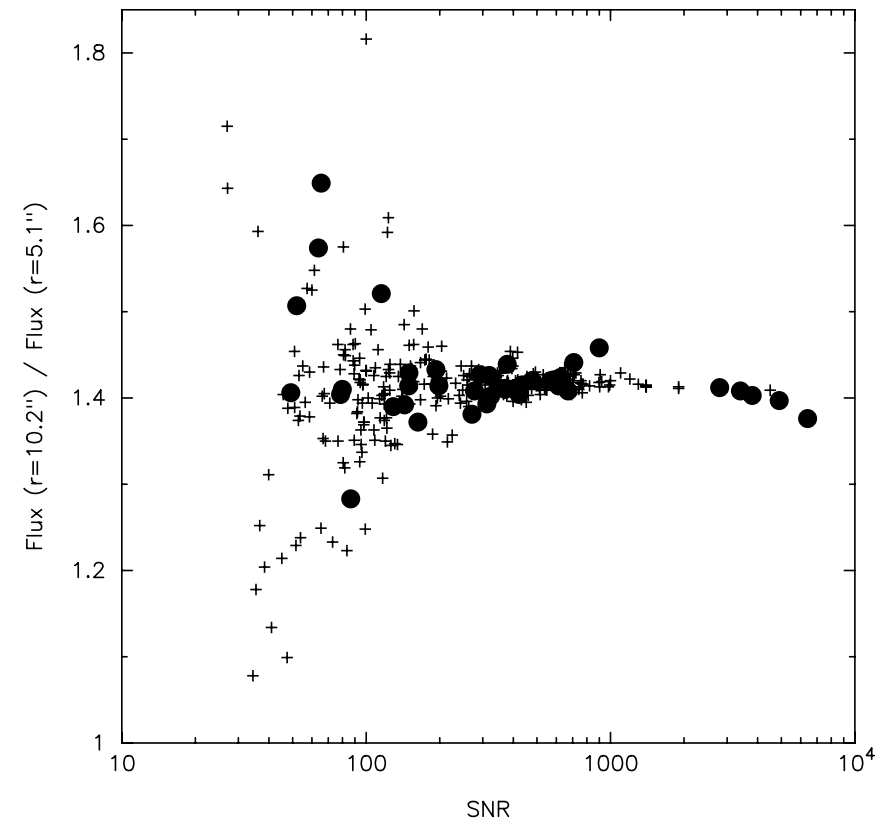

FIG. 6.- Ratio of the $24 \mu \mathrm{m}$ flux density measured in a $10.2^{\prime \prime}$ radius aperture (=4 pixels) to that in a 5.1" radius aperture (=2 pixels) as a function of the $\mathrm{S} / \mathrm{N}$ of the $24 \mu \mathrm{m}$ PRF photometry. Filled circles represent sources with a $\geq 3 \sigma 24 \mu \mathrm{m}$ excess confirmed by the IRS spectrum, and crosses indicate sources without detectable $24 \mu \mathrm{m}$ excesses (Carpenter et al. 2008).

just under 1.8. The $70 \mu \mathrm{m}$ emission for this object is centered on the stellar position to within $3^{\prime \prime}$, and the observed $70 \mu \mathrm{m}$ emission is consistent with the expected stellar photosphere.

In summary, we conclude that the astrometry and curve-ofgrowth for most sources are consistent with point source emission centered on the stellar position. No compelling evidence exists

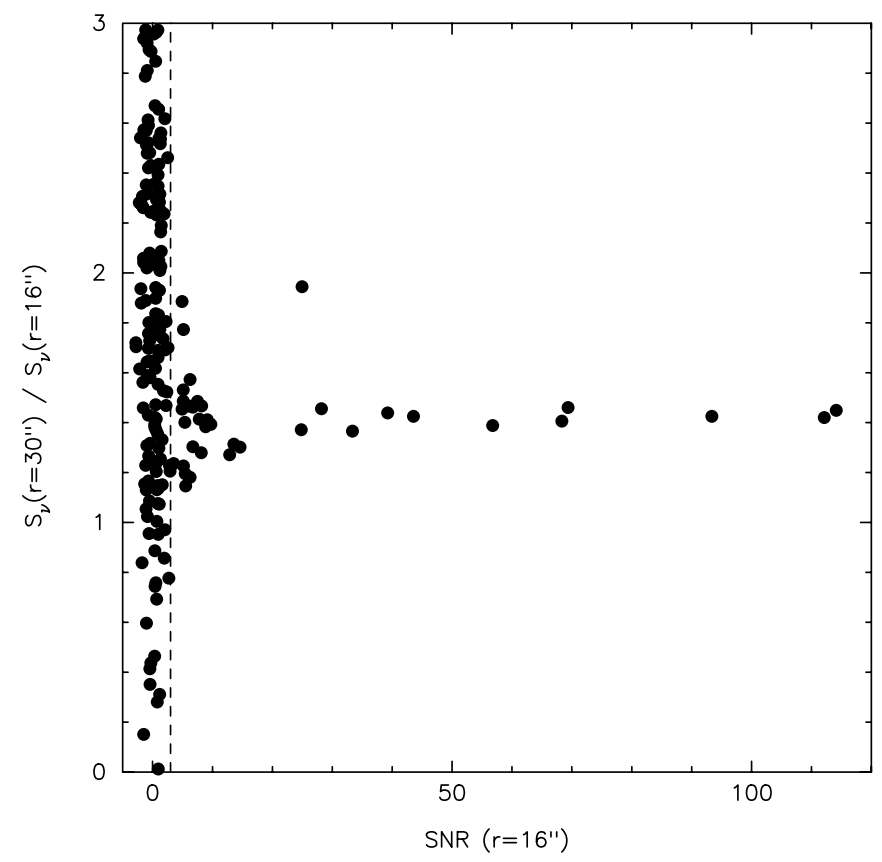

FIG. 7.- Ratio of the $70 \mu \mathrm{m}$ flux density measured in a $30^{\prime \prime}$ radius aperture to that in a $16^{\prime \prime}$ radius aperture as a function of the signal to noise ratio. The vertical dashed line is drawn at $\mathrm{S} / \mathrm{N}=3$. The two sources with $\mathrm{S} / \mathrm{N}>3$ and flux density ratios greater than 1.8 have a nearby source that partially overlap the source aperture. These two contaminating sources were PRF-subtracted before performing the final photometry.

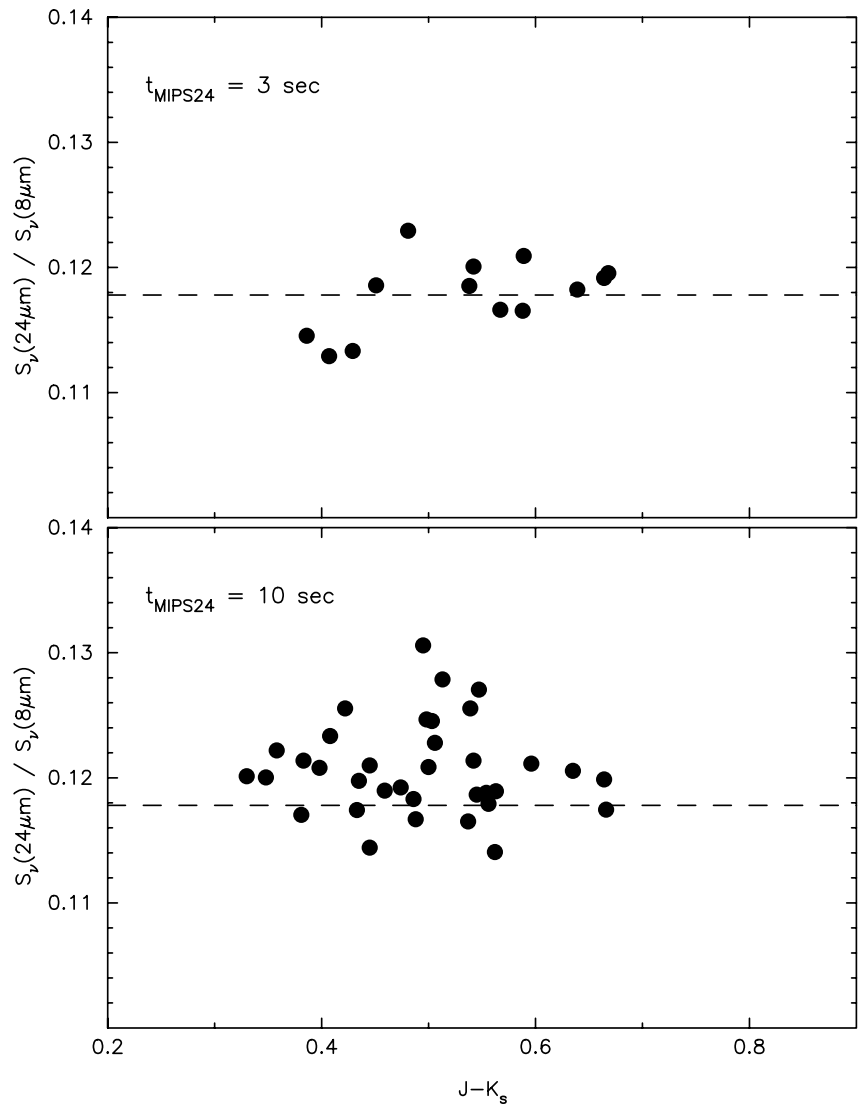

FIG. 8.- Ratio of 24 to $8 \mu \mathrm{m}$ flux densities $\left(R_{24 / 8}\right)$ plotted as a function of the $J-K_{s}$ color for stars observed with a $0.10 \mathrm{~s}$ IRAC frame time that do not have a IRS excess (see text for a complete description of the selection criteria). The top panel shows the results for stars observed with a MIPS $24 \mu \mathrm{m}$ exposure time of $3 \mathrm{~s}$, and the bottom panel for $10 \mathrm{~s}$ exposure time. The dashed line shows the mean flux ratios for the $3 \mathrm{~s}$ MIPS data. The ratio of the mean value of $R_{24 / 8}$ in the $3 \mathrm{~s}$ MIPS data to the $10 \mathrm{~s}$ data is $0.976 \pm 0.008$.

that contaminants systematically influence the 24 photometry. At $70 \mu \mathrm{m}$, contaminants needed to be removed for a few sources before measuring the final photometry, and the sources are noted in Table 1. These results do not exclude the possibility that the photometry for some sources may be contaminated, but any such contamination must be present in a minority of sources.

\section{CROSS-INSTRUMENT CALIBRATION}

In this section we use the FEPS data to examine the crossinstrument calibration. We first analyze the 24 to $8 \mu \mathrm{m}$ flux density ratio, which plays a prominent role in a companion paper to identify sources that exhibit excess emission from circumstellar dust (Carpenter et al. 2008). We then compare the IRS and MIPS $24 \mu \mathrm{m}$ calibration.

\subsection{IRAC $8 \mu \mathrm{m}$ vs. MIPS $24 \mu \mathrm{m}$}

The observed IRAC $8 \mu \mathrm{m}$ flux density is consistent with photospheric emission for most stars in the FEPS sample (Carpenter et al. 2008). The $24 / 8 \mu \mathrm{m}$ flux ratio then is diagnostic of sources that exhibit circumstellar dust emission at $24 \mu \mathrm{m}$. The precision to which this ratio can identify excesses depends on the relative calibration stability of the IRAC and MIPS instruments over time and between the various observing modes.

The primary difference between observations of different stars is the exposure time for individual IRAC and MIPS images. We 


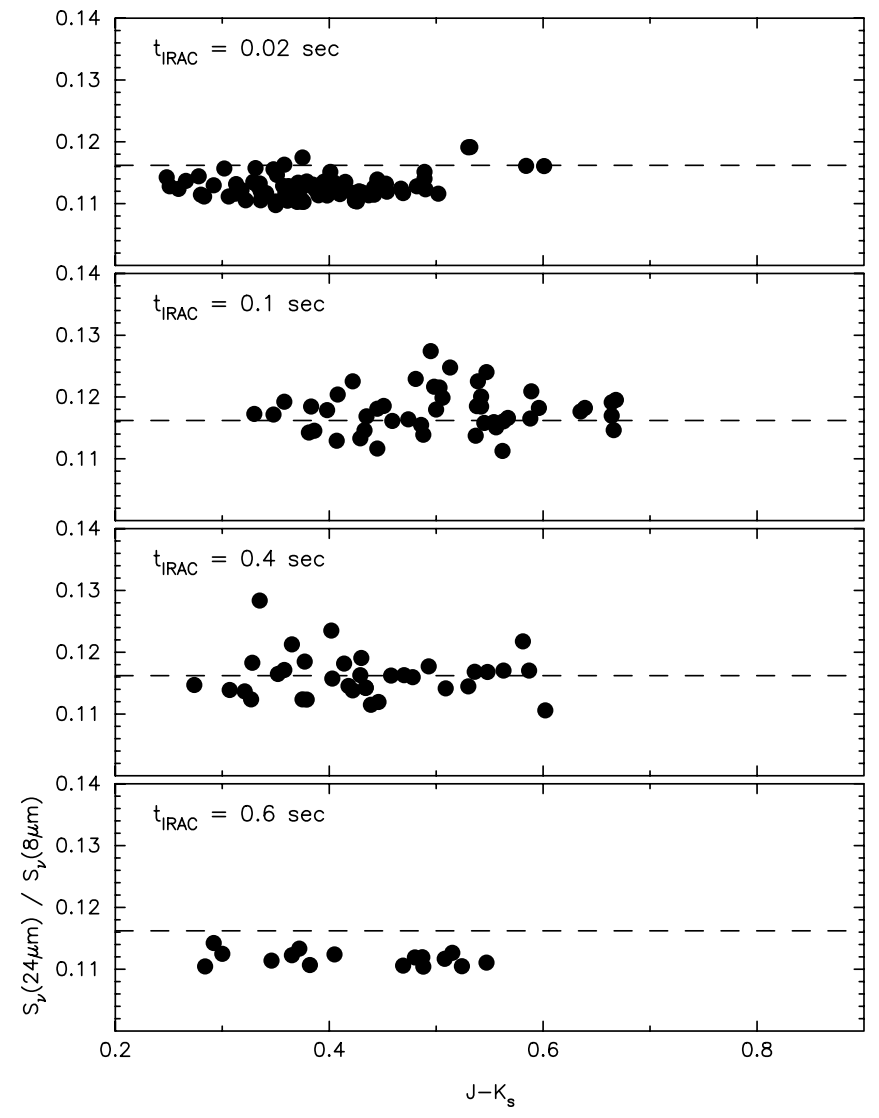

FIG. 9.-Ratio of 24 to $8 \mu \mathrm{m}$ flux densities plotted vs. dereddened $J-K_{s}$ color for IRAC frame times of $0.02,0.1,0.4$, and $0.6 \mathrm{~s}$. The dashed line in each panel shows the mean flux ratio for the $0.4 \mathrm{~s}$ IRAC data. These results suggest that the observed $24 / 8 \mu \mathrm{m}$ flux ratio varies with IRAC frame time.

first examine the relative stability of the MIPS $24 \mu \mathrm{m}$ calibration by selecting stars in the FEPS program that were observed with the same IRAC frame time, but different MIPS exposure times. We selected 48 stars in the FEPS sample that (1) have been observed with IRAC frame times of $0.10 \mathrm{~s}$, (2) do not show evidence for more than a $2 \sigma$ infrared excesses in the FEPS IRS spectra (Carpenter et al. 2008) to ensure the $24 \mu \mathrm{m}$ emission is from the photosphere, (3) the variation in the encircled energy with aperture radius in the IRAC images is consistent with a point source (see $\S 2.2$ ), and (4) the dereddened $J-K_{s}$ color is less than 0.7 mag to remove the intrinsically reddest stars in the FEPS sample. We used a $0.10 \mathrm{~s}$ IRAC frame time to obtain the largest sample of stars observed with different MIPS exposure times.

In Figure 8 we plot the $24 / 8 \mu$ m flux density ratio $\left(\equiv R_{24 / 8}\right)$ for MIPS $3 \mathrm{~s}$ (top panel) and $10 \mathrm{~s}$ (bottom panel) exposure times versus the dereddened 2 MASS $J-K_{S}$ color using the extinction estimates derived in $\S$ B.1. The two sample of stars span similar ranges of dereddened $J-K_{s}$ colors, and we assume that the two samples also share the same intrinsic photospheric [8] - [24] color. The Shapiro-Wilk statistic $\left(\equiv p_{\mathrm{SW}}\right)$ indicates that the distribution of observed data points about the mean is consistent with a normal distribution for each sample $\left(p_{\mathrm{SW}}=0.76\right.$ for the $3 \mathrm{~s}$ MIPS data and $p_{\mathrm{SW}}=0.31$ for the $10 \mathrm{~s}$ data). The Student's $t$-test then can be used to compare the mean values of $R_{24 / 8}$ for the 3 and 10 s MIPS data. The probability from the $t$-test that the mean values of $R_{24 / 8}$ for the two samples are consistent with each other is 0.009 . The ratio of the mean value of $R_{24 / 8}$ in a $3 \mathrm{~s}$ MIPS exposure to that in a $10 \mathrm{~s}$ exposure is $0.976 \pm 0.008$, where the uncertainty was computed

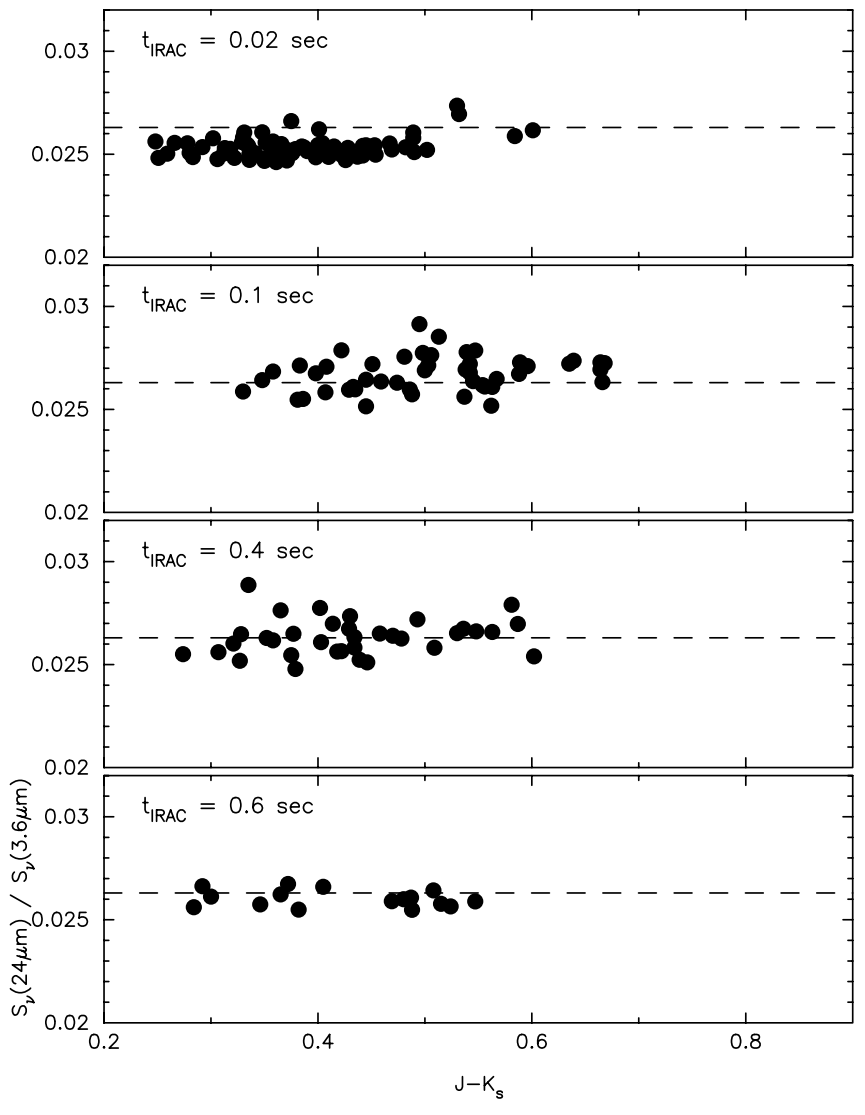

FIG. 10.- Same as Fig. 9, but for the IRAC $3.6 \mu \mathrm{m}$ band.

as the standard deviation of the mean. These results suggest that the mean $R_{24 / 8}$ value is higher for the $10 \mathrm{~s}$ MIPS data on average compared to the $3 \mathrm{~s}$ data.

Engelbracht et al. (2007) measured directly any MIPS $24 \mu \mathrm{m}$ calibration offsets by observing a sample of 11 stars with 3,10 , and $30 \mathrm{~s}$ MIPS exposure times. They also found that the measured flux densities were larger on average in $10 \mathrm{~s}$ exposure data compared to $3 \mathrm{~s}$ observations. However, the magnitude of their offset (1\%) is 2.4 times smaller than the offset derived from the FEPS data. While the reduction procedure adopted here attempted to follow that recommended by Engelbracht et al. (2007) our data processing was nonetheless performed using SSC products and custom software that could account for the different results. Also, we adopted PRF-fitting photometry, while Engelbracht et al. (2007) used aperture photometry. As a check of our data reduction methods, we compared our photometry with the results from Rieke et al. (2008), who used the pipeline described in Engelbracht et al. (2007) to process data for 31 FEPS sources that were observed with $3 \mathrm{~s}$ exposure times. For these 31 stars, the median difference between the flux densities measured by FEPS and Rieke et al. (2008) is $0.0 \%$ with a dispersion of $2.6 \%$. Therefore our data reduction procedures for at least the $3 \mathrm{~s}$ exposure data yields photometry consistent with the Engelbracht et al. (2007) processing, but no independent check is available for the $10 \mathrm{~s}$ MIPS data.

We now consider the relative flux calibration for stars with different IRAC exposure times. In Figure 9 we plot the $24 / 8 \mu \mathrm{m}$ flux density ratio versus dereddened $J-K_{s}$ color for stars observed with various IRAC frame times. The MIPS $24 \mu \mathrm{m}$ photometry obtained with $10 \mathrm{~s}$ exposure times have been scaled by a factor of 0.976 based on the analysis above since the MIPS calibration is tied mainly to data obtained with $3 \mathrm{~s}$ exposure times 


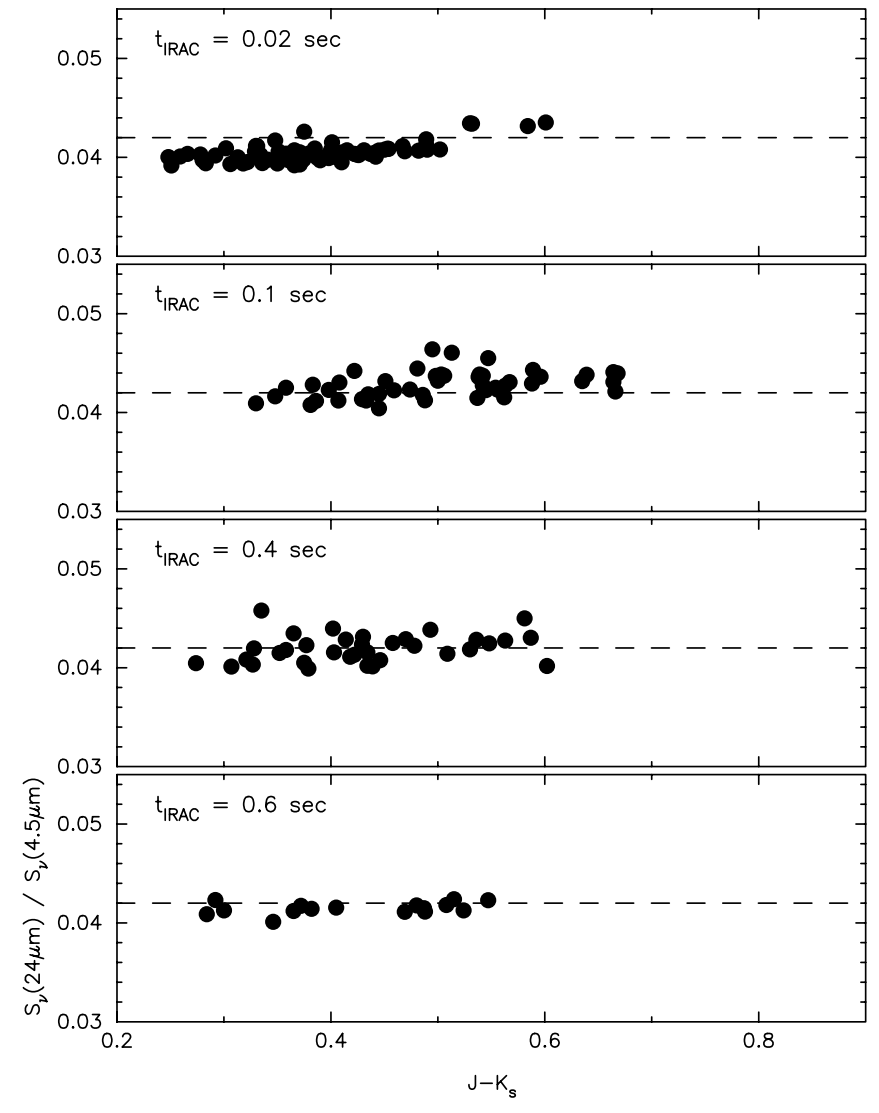

FIG. 11.- Same as Fig. 9, but for the IRAC $4.5 \mu \mathrm{m}$ band.

(Engelbracht et al. 2007). As shown in the figure, systematic differences are present in the mean flux density ratio between the various IRAC frame times. Offsets are present even if the $10 \mathrm{~s}$ MIPS $24 \mu \mathrm{m}$ data are not scaled, but the magnitude of the offset changes. We adopt the $0.4 \mathrm{~s}$ frame time as the fiducial calibration since the calibration of the $0.4 \mathrm{~s}$ subarray data and the full-array data are the same to within $1 \%$ (see $\S 2.2$ ). A multiplicative scale factor of $0.971 \pm 0.005$ must be applied to the $0.02 \mathrm{~s}$ IRAC frame-time data to force agreement with the $0.4 \mathrm{~s}$ data, $1.014 \pm 0.007$ for the $0.1 \mathrm{~s}$ data, and $0.962 \pm 0.006$ for the $0.6 \mathrm{~s}$ data, where the uncertainties are the standard deviation of the mean. In Figures 10 and 11 we present a similar analysis for the IRAC 3.6 and $4.5 \mu \mathrm{m}$ bands which demonstrates that offsets are also present in these bands. Since only five FEPS stars were observed in the IRAC $5.8 \mu \mathrm{m}$ band, we were unable to derive offsets for that band.

Table 2 summarizes the multiplicative factors that must be applied to the flux ratios as a function of frame time to scale the calibration to the $0.4 \mathrm{~s}$ frame time data. The offsets are similar in the three bands for a given frame time, although the offset in the $0.6 \mathrm{~s}$ frame-time data may be larger for IRAC $8 \mu \mathrm{m}$ than in the 3.6 and

TABLE 2

IRAC Calibration Offsets Relative to 0.40 s Frame Time

\begin{tabular}{|c|c|c|c|}
\hline \multirow{2}{*}{$\begin{array}{l}\text { BAND } \\
(\mu \mathrm{m})\end{array}$} & \multicolumn{3}{|c|}{ IRAC FRAME TIME } \\
\hline & $0.02 \mathrm{~s}$ & $0.10 \mathrm{~s}$ & $0.60 \mathrm{~s}$ \\
\hline $3.6 \ldots \ldots \ldots \ldots \ldots \ldots$ & $0.961 \pm 0.006$ & $1.014 \pm 0.007$ & $0.988 \pm 0.007$ \\
\hline 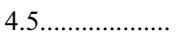 & $0.964 \pm 0.006$ & $1.021 \pm 0.007$ & $0.988 \pm 0.007$ \\
\hline 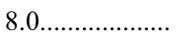 & $0.971 \pm 0.005$ & $1.014 \pm 0.007$ & $0.962 \pm 0.006$ \\
\hline
\end{tabular}

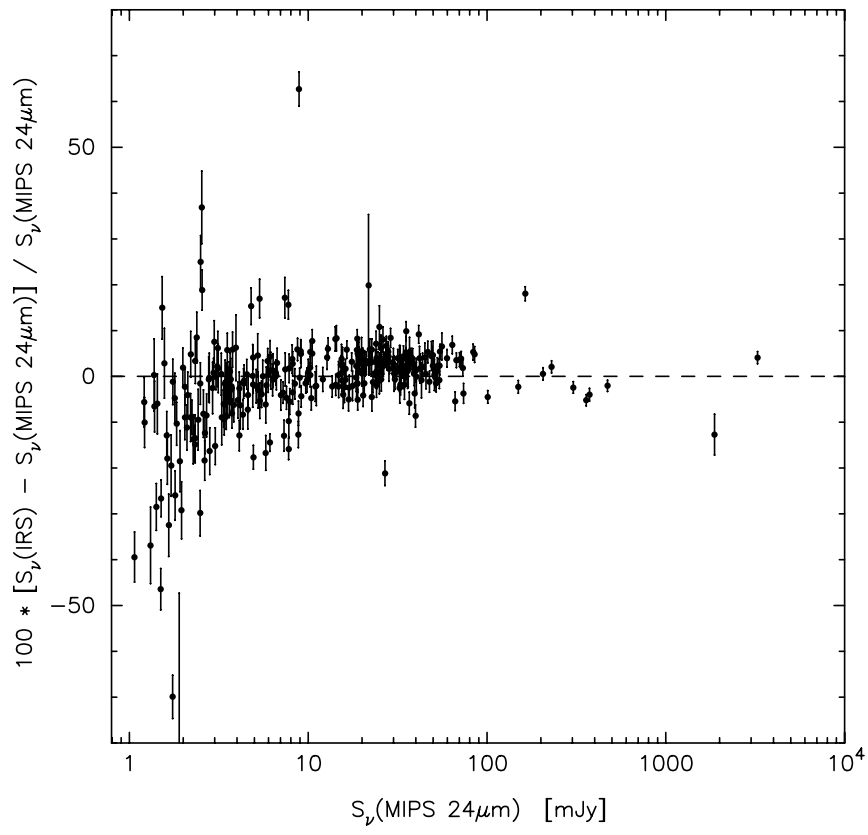

FIG. 12.-Percent difference between the synthetic IRS $24 \mu$ m photometry and MIPS $24 \mu \mathrm{m}$ photometry as a function of the MIPS $24 \mu \mathrm{m}$ flux density. The IRS $24 \mu \mathrm{m}$ photometry was computed by integrating the observed IRS spectrum over the MIPS $24 \mu \mathrm{m}$ bandpass. The horizontal dashed line is shown for reference.

$4.5 \mu \mathrm{m}$ bands. We consider these correction factors preliminary since they have not yet been verified by observing the same star with different frame times. No corrections for any integrationdependent calibrations have been applied to the photometry in Table 1, but the frame times are listed to enable the corrections to be applied by the reader.

\subsection{IRS vs. MIPS $24 \mu \mathrm{m}$}

The IRS spectral coverage encompasses the spectral response of the MIPS $24 \mu \mathrm{m}$ bandpass. To compare the relative calibration of the two instruments, we computed synthetic $24 \mu \mathrm{m}$ photometry from the IRS spectrum and the MIPS $24 \mu \mathrm{m}$ spectral response using the procedure described in Appendix C2.

In Figure 12 we plot the percent difference between the IRS synthetic photometry and MIPS $24 \mu \mathrm{m}$ photometry as a function of the MIPS $24 \mu \mathrm{m}$ flux density. No exposure-time-dependent corrections have been applied to the MIPS $24 \mu \mathrm{m}$ flux densities for this analysis. For sources brighter than $10 \mathrm{mJy}$, which have the highest $\mathrm{S} / \mathrm{N}$, the median difference in the $24 \mu \mathrm{m}$ flux densities between the IRS spectra and the MIPS photometry is $2.1 \%$. The median difference for sources between 3 and $10 \mathrm{mJy}$ is $-1.6 \%$. These differences are within the $1 \sigma$ calibration uncertainty for both MIPS (4\%; Engelbracht et al. 2007) and IRS (>5\%; Infrared Spectrograph Data Handbook ver. 3.1). However, for individual sources, the difference between the MIPS and IRS flux densities are larger than expected based on the quantifiable internal uncertainties. One significant discrepancy is ScoPMS 52, where the IRS $24 \mu$ m flux density is $63 \%$ higher than the MIPS $24 \mu \mathrm{m}$ flux density. Inspection of the MIPS $24 \mu \mathrm{m}$ image shows that there is a source $18^{\prime \prime}$ away that is an order of magnitude brighter than ScoPMS 52 (see Bouwman et al. 2008), and this source likely contributes flux to the IRS spectrum.

\section{SUMMARY}

The FEPS Spitzer Legacy program was designed to obtain infrared photometry from 3.6 to $160 \mu \mathrm{m}$ and low-resolution spectra 
from 5 to $35 \mu \mathrm{m}$ for 328 solar-type stars spanning ages from 3 Myr to 3 Gyr. The broad goal of FEPS was to determine the incidence of circumstellar disks and place the results in context with the expected evolution of our solar system. An essential component of this study was to construct carefully calibrated SEDs. Here, we outline the data reduction procedures adopted by the FEPS team to obtain accurate and well-characterized Spitzer photometry and spectra.

The adopted image processing steps for the IRAC, MIPS, and IRS data closely follow the recommended procedures by the Spitzer Science Center and Spitzer Instrument Teams. We describe in detail the data reduction methods for each instrument and the procedures used to validate the data products. We present in Table 1 the measured IRAC (3.6, 4.5, and $8 \mu \mathrm{m}$ bands) and MIPS (24 and $70 \mu \mathrm{m}$ ) flux densities and uncertainties. The extracted, calibrated IRS spectra are available electronically.
J. M. C. thanks Dave Frayer, Sean Carey, Bill Reach, Jason Surace, and the staff at the Spitzer Science Center for patiently answering numerous questions regarding Spitzer data. We are grateful to the anonymous referee and George Rieke for providing valuable comments. We also thank Debbie Padgett, Tim Brooke, Dan Watson, Pat Morris, and the rest of the FEPS team for their many contributions throughout this project. This work is based on observations made with the Spitzer Space Telescope, which is operated by JPL/Caltech under a contract with NASA. The program made use of data and resources from the FEPS project, which receives support from NASA contracts 1224768, 1224634, and 1224566 administered through JPL. This research made use of the SIMBAD database, operated at CDS, Strasbourg, France, as well as data products from the Two Micron All Sky Survey, which is a joint project of the University of Massachusetts and the Infrared Processing and Analysis Center/Caltech, funded by NASA and the NSF.

\section{APPENDIX A}

\section{STELLAR PHOTOMETRY}

The FEPS team obtained optical photometry in the BVRI broadband filters for 45 stars. Observations were obtained with the $61^{\prime \prime}$ Kuiper Telescope on 2003 May 8 and 2003 September 29-30, and the CTIO 0.9 m telescope on 2004 March $18-21$.

The Kuiper observations used a $2048 \times 2048$ pixel CCD with a pixel scale of $0.45^{\prime \prime} \mathrm{pixel}^{-1}$. Images were processed by subtracting the bias, dividing by a "master" flat field created from sky observations to remove large-scale response variations over the CCD, and dividing by a dome flat to remove pixel-to-pixel variations. The CTIO observations were performed with a $2048 \times 2048$ CCD and a pixel scale of $0.40^{\prime \prime}$ pixel $^{-1}$. The CCD is read out with different amplifiers for each quadrant. Each quadrant was bias subtracted and divided by dome flats.

Photometry was measured using aperture photometry with a sky annulus that extended from 20 to 30 pixels, and an aperture radius of 11 and 12 pixels for the CTIO and Kuiper images, respectively. Eight FEPS stars had a nearby source in projection, and a smaller aperture radius between 2-3 pixels to isolate the photometry to the FEPS target. The observations were calibrated by observing multiple standard stars from Landolt (1992) to solve for the air mass coefficient, the photometric zero point, and color terms to place the photometry on the Johnson-Cousins photometric system. Total photometric uncertainties were computed as the rms sum of internal photometric uncertainties, the zero point, and the color terms. The photometry for the 45 sources are presented in Table 3.

\section{APPENDIX B}

\section{STELLAR PROPERTIES}

In this section we describe the procedure to assign estimates of the visual extinction, surface gravity, metallicity, and effective temperature for each star in the FEPS sample. These derived parameters were used in several FEPS studies, and served as initial estimates for the Kurucz model fitting (see Appendix C).

\section{B1. VISUAL EXTINCTION}

Distances to the FEPS targets extend upwards of $343 \mathrm{pc}$ and the extinction from the interstellar medium may be nonnegligible. The visual extinction toward individual stars was estimated from one of the following techniques in priority order: (1) proximity within the Local Bubble, (2) as a member of stellar cluster that has been extensively studied previously; (3) color excess at optical and nearinfrared wavelengths; and (4) a galactic extinction model. We now describe each of these techniques.

Stars within the Local Bubble are expected to have small extinction at visual wavelengths. The size of the Local Bubble has been measured by observing interstellar absorption lines toward stars with known distances, and then determining the column density as a function of distance. Welsh et al. (1998) present an analysis of Na I column density measurements toward stars with Hipparcos distance estimates, and they found that the visual extinction is less than 0.01 mag out to a distance of $d=75$ pc. We adopted an extinction of 0 mag for the 169 stars in the FEPS sample where $d+3 \Delta d \leq 75 \mathrm{pc}$, where $\Delta d$ is the $1 \sigma$ distance uncertainty (see Meyer et al. 2006 for a discussion on the distance determinations).

The visual extinction toward the clusters in the FEPS sample has been extensively studied in the literature. For the Hyades, Taylor (2006) place an upper limit at $95 \%$ confidence of $E(B-V)=0.001 \mathrm{mag}$, and we adopt $A_{V}=0$ mag. Breger (1986) compiled spectral types and optical photometry for about 120 Pleiades members and derived $E(B-V)=0.04$ on average, but with lower reddening to the east of the cluster $(0.03 \mathrm{mag})$ compared to the west $(0.06 \mathrm{mag})$. Assuming a factor of 3.1 to convert the $B-V$ reddening to visual extinction, we adopt a constant value of 0.12 mag for the Pleiades stars. Following Pinsonneault et al. (1998), we adopt an average $E(B-V)=0.10$ (see Crawford \& Barnes 1974; Prosser 1992), or $A_{V}=0.31 \mathrm{mag}$, for Alpha Per. For IC 2602, we adopt a visual extinction of 0.12 mag (Whiteoak 1961).

Many of the FEPS stars are field objects that have distances greater than $75 \mathrm{pc}$. The visual extinction for these stars was computed from the color excess given the published spectral types (see Meyer et al. 2006) and observed colors. Optical (Johnson $B$ and $V$, Tycho $B_{T}$ and $V_{T}$ ) 
TABLE 3

New BVRI Photometry For FEPS Sources

\begin{tabular}{|c|c|c|c|c|}
\hline Source & $B_{\mathrm{J}}$ & $V_{\mathrm{J}}$ & $R_{\mathrm{C}}$ & $I_{\mathrm{C}}$ \\
\hline 1E $0307.4+1424 .$. & $11.129 \pm 0.030$ & $10.463 \pm 0.025$ & $10.045 \pm 0.023$ & $9.693 \pm 0.023$ \\
\hline 1E 0324.1-2012 & $11.049 \pm 0.037$ & $10.435 \pm 0.031$ & $10.082 \pm 0.028$ & $9.726 \pm 0.024$ \\
\hline 1RXS J025216.9+361658 ….............. & $11.839 \pm 0.031$ & $10.715 \pm 0.026$ & $10.016 \pm 0.019$ & $9.369 \pm 0.024$ \\
\hline 1RXS J025751.8+115759................... & $11.645 \pm 0.031$ & $10.797 \pm 0.026$ & $10.266 \pm 0.024$ & $9.799 \pm 0.024$ \\
\hline 1RXS J031644.0+192259 ................... & $11.598 \pm 0.030$ & $10.996 \pm 0.025$ & $10.598 \pm 0.023$ & $10.248 \pm 0.023$ \\
\hline 1RXS J031907.4+393418_................... & $12.444 \pm 0.030$ & $11.653 \pm 0.025$ & $11.159 \pm 0.023$ & $10.734 \pm 0.023$ \\
\hline 1RXS J035028.0+163121 ….................. & $11.257 \pm 0.030$ & $10.567 \pm 0.025$ & $10.096 \pm 0.023$ & $9.701 \pm 0.023$ \\
\hline 1RXS J051111.1+281353 …................. & $11.492 \pm 0.053$ & $10.522 \pm 0.028$ & $9.933 \pm 0.030$ & $9.398 \pm 0.028$ \\
\hline 1RXS J053650.0+133756 ................... & $11.482 \pm 0.054$ & $10.551 \pm 0.028$ & $9.994 \pm 0.032$ & $9.496 \pm 0.028$ \\
\hline 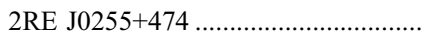 & $11.773 \pm 0.033$ & $10.723 \pm 0.027$ & $10.052 \pm 0.027$ & $9.497 \pm 0.026$ \\
\hline BPM $87617 \ldots \ldots \ldots$. & $12.013 \pm 0.037$ & $10.849 \pm 0.025$ & $10.154 \pm 0.016$ & $9.486 \pm 0.016$ \\
\hline HD $279788 \ldots$ & $11.452 \pm 0.030$ & $10.640 \pm 0.025$ & $10.137 \pm 0.023$ & $9.704 \pm 0.023$ \\
\hline HD 286264 . & $12.050 \pm 0.055$ & $10.889 \pm 0.023$ & $10.144 \pm 0.033$ & $9.451 \pm 0.028$ \\
\hline MML $18 \ldots \ldots$. & $11.790 \pm 0.004$ & $10.803 \pm 0.004$ & $10.231 \pm 0.004$ & $9.697 \pm 0.004$ \\
\hline 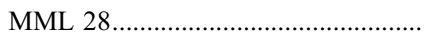 & $12.385 \pm 0.005$ & $11.347 \pm 0.006$ & $10.753 \pm 0.004$ & $10.203 \pm 0.005$ \\
\hline 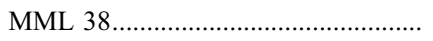 & $11.786 \pm 0.003$ & $10.957 \pm 0.004$ & $10.454 \pm 0.003$ & $9.954 \pm 0.004$ \\
\hline 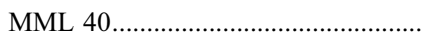 & $11.527 \pm 0.003$ & $10.665 \pm 0.005$ & $10.161 \pm 0.003$ & $9.679 \pm 0.005$ \\
\hline 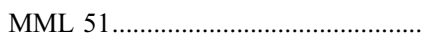 & $12.203 \pm 0.005$ & $11.131 \pm 0.005$ & $10.492 \pm 0.005$ & $9.884 \pm 0.005$ \\
\hline [PZ99] J155847.8-175800 ............... & $13.164 \pm 0.046$ & $11.875 \pm 0.031$ & $11.152 \pm 0.020$ & $10.403 \pm 0.019$ \\
\hline [PZ99] J160814.7-190833 ................... & $12.593 \pm 0.066$ & $11.458 \pm 0.032$ & $10.829 \pm 0.021$ & $10.241 \pm 0.021$ \\
\hline [PZ99] J161318.6-221248................. & $11.493 \pm 0.045$ & $10.397 \pm 0.030$ & $9.783 \pm 0.020$ & $9.165 \pm 0.019$ \\
\hline [PZ99] J161329.3-231106 ................. & $12.836 \pm 0.045$ & $11.693 \pm 0.031$ & $11.033 \pm 0.020$ & $10.339 \pm 0.020$ \\
\hline [PZ99] J161402.1-230101 ................. & $12.379 \pm 0.046$ & $11.353 \pm 0.031$ & $10.777 \pm 0.020$ & $10.197 \pm 0.020$ \\
\hline [PZ99] J161411.0-230536 ................. & $11.824 \pm 0.046$ & $10.671 \pm 0.032$ & $10.025 \pm 0.021$ & $9.377 \pm 0.020$ \\
\hline [PZ99] J161459.2-275023 ................. & $12.133 \pm 0.048$ & $11.194 \pm 0.033$ & $10.666 \pm 0.021$ & $10.153 \pm 0.021$ \\
\hline [PZ99] J161618.0-233947 ................. & $11.603 \pm 0.047$ & $10.659 \pm 0.032$ & $10.126 \pm 0.021$ & $9.593 \pm 0.021$ \\
\hline RX J0258.4+2947 … & & $11.378 \pm 0.039$ & $10.853 \pm 0.033$ & $10.361 \pm 0.033$ \\
\hline RX J0331.1+0713 & $11.610 \pm 0.031$ & $10.719 \pm 0.026$ & $10.145 \pm 0.024$ & $9.608 \pm 0.024$ \\
\hline RX J0357.3+1258 & $11.691 \pm 0.031$ & $10.994 \pm 0.026$ & $10.547 \pm 0.023$ & $10.132 \pm 0.024$ \\
\hline RX J0434.3+0226 & $13.557 \pm 0.028$ & $12.554 \pm 0.020$ & $11.897 \pm 0.016$ & $11.253 \pm 0.019$ \\
\hline RX J0442.5+0906 & $11.974 \pm 0.056$ & $11.186 \pm 0.029$ & $10.720 \pm 0.033$ & $10.301 \pm 0.029$ \\
\hline 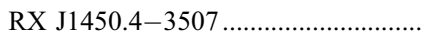 & $11.578 \pm 0.004$ & $10.651 \pm 0.003$ & $10.091 \pm 0.002$ & $9.538 \pm 0.003$ \\
\hline 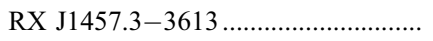 & $10.966 \pm 0.004$ & $10.201 \pm 0.003$ & $9.748 \pm 0.002$ & $9.332 \pm 0.003$ \\
\hline 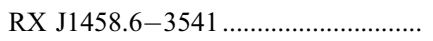 & $11.762 \pm 0.005$ & $10.724 \pm 0.003$ & $10.089 \pm 0.002$ & $9.476 \pm 0.003$ \\
\hline 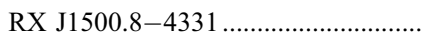 & $11.960 \pm 0.012$ & $11.099 \pm 0.004$ & $10.584 \pm 0.004$ & $10.074 \pm 0.006$ \\
\hline RX J1518.4-3738 & $11.688 \pm 0.012$ & $10.826 \pm 0.004$ & $10.313 \pm 0.004$ & $9.817 \pm 0.006$ \\
\hline 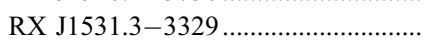 & $11.687 \pm 0.012$ & $10.874 \pm 0.004$ & $10.391 \pm 0.004$ & $9.937 \pm 0.006$ \\
\hline RX J1541.1-2656 & $12.099 \pm 0.013$ & $11.210 \pm 0.005$ & $10.658 \pm 0.006$ & $10.168 \pm 0.007$ \\
\hline 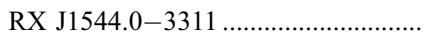 & $11.868 \pm 0.009$ & $10.953 \pm 0.005$ & $10.411 \pm 0.004$ & $9.858 \pm 0.007$ \\
\hline RX J1600.6-2159 & $12.140 \pm 0.045$ & $11.088 \pm 0.030$ & $10.518 \pm 0.019$ & $9.962 \pm 0.019$ \\
\hline RX J1839.0-3726 & $12.035 \pm 0.010$ & $11.092 \pm 0.006$ & $10.529 \pm 0.005$ & $9.996 \pm 0.009$ \\
\hline 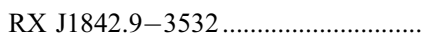 & $12.895 \pm 0.010$ & $11.998 \pm 0.006$ & $11.313 \pm 0.004$ & $10.653 \pm 0.008$ \\
\hline 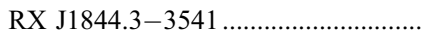 & $12.213 \pm 0.008$ & $11.184 \pm 0.005$ & $10.555 \pm 0.004$ & $9.940 \pm 0.007$ \\
\hline RX J1852.3-3700 & $13.343 \pm 0.008$ & $12.219 \pm 0.005$ & $11.510 \pm 0.004$ & $10.810 \pm 0.007$ \\
\hline W79 & $12.304 \pm 0.004$ & $11.499 \pm 0.004$ & $11.043 \pm 0.004$ & $10.638 \pm 0.004$ \\
\hline
\end{tabular}

and near-infrared (2MASS $J, H$, and $K_{S}$ ) photometry were compiled from the literature (see Appendix C1) or measured by the FEPS team (see Appendix A). The intrinsic colors as a function of spectral type were compiled from the literature by cross-correlating the Hipparcos catalog with the Michigan Spectral Catalog, Tycho-2, and 2MASS. The positional match between the Tycho-2 and Michigan spectral atlas from Wright et al. (2003) was used as a starting point. Only 2MASS sources with a PH_QUAL flag of AAA and a confusion flag of 000 were used. A photometric uncertainty of less $\leq 0.072 \mathrm{mag}$ (i.e., signal-to-noise ratio $>15$ ) was required in each photometric band. The average color was then computed as a function of spectral type for stars within $75 \mathrm{pc}$ for $B$ and $V$ photometry, and within $100 \mathrm{pc}$ for colors involving $J, H$, and $K_{s}$. In computing the average colors, individual measurements were weighted by the inverse variance of the measurements, and outliers from poor photometry or spectral types were removed in an iterative sigma-clipping procedure. Table 4 lists the adopted intrinsic colors for the relevant spectral types in the FEPS sample, the dispersion in the observed colors, and the number of stars that met the above criteria. Color excesses were computed from the observed $(B-V)_{\text {Johnson }},(B-V)_{\text {Tycho }}, V_{\text {Tycho }}-K_{s}$, and $J-K_{s}$ colors and the intrinsic colors listed in Table 4. Intrinsic $(B-V)_{\text {Johnson }}$ colors were computed from the $(B-V)_{\text {Tycho }}$ colors and the Tycho-toJohnson transformation equations in Mamajek et al. $(2002,2006)$. The visual extinction was estimated for each color using the extinction law compiled by Mathis (1990) and the weighted mean was adopted as the extinction.

For nine stars, the visual extinction could not be estimated with the above techniques since either a spectral type was not available, or the computed extinction was unphysical (i.e., $A_{V}<0 \mathrm{mag}$ ). In the latter case, it is presumed that the photometry was poor or the spectral type is erroneous. For these stars, we estimated the extinction using the Sandage (1972) extinction model assuming an exponential disk (see 
TABLE 4

Adopted InTRINSIC Colors

\begin{tabular}{|c|c|c|c|c|c|c|c|c|c|}
\hline \multirow[b]{2}{*}{ Spectral Type } & \multicolumn{3}{|c|}{$(B-V)_{T}$} & \multicolumn{3}{|c|}{$J-K_{s}$} & \multicolumn{3}{|c|}{$V_{T}-K_{s}$} \\
\hline & Average & Dispersion & $N$ & Average & Dispersion & $N$ & Average & Dispersion & $N$ \\
\hline F2 ………… & 0.383 & 0.028 & 74 & 0.224 & 0.029 & 153 & 0.980 & 0.093 & 58 \\
\hline F3 ………… & 0.433 & 0.025 & 147 & 0.237 & 0.019 & 257 & 1.090 & 0.057 & 102 \\
\hline F5 ......................... & 0.483 & 0.022 & 266 & 0.267 & 0.026 & 491 & 1.216 & 0.069 & 230 \\
\hline 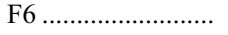 & 0.527 & 0.022 & 214 & 0.288 & 0.020 & 337 & 1.305 & 0.058 & 173 \\
\hline F7 ………… & 0.558 & 0.026 & 250 & 0.303 & 0.025 & 371 & 1.354 & 0.050 & 177 \\
\hline F8 & 0.592 & 0.023 & 130 & 0.320 & 0.025 & 211 & 1.393 & 0.043 & 90 \\
\hline G0 & 0.632 & 0.025 & 206 & 0.329 & 0.021 & 313 & 1.460 & 0.041 & 145 \\
\hline 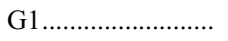 & 0.648 & 0.023 & 114 & 0.342 & 0.025 & 197 & 1.498 & 0.067 & 117 \\
\hline G2 & 0.669 & 0.025 & 167 & 0.350 & 0.024 & 248 & 1.554 & 0.069 & 166 \\
\hline G3 & 0.707 & 0.033 & 324 & 0.371 & 0.029 & 521 & 1.601 & 0.047 & 241 \\
\hline G5 & 0.754 & 0.029 & 331 & 0.387 & 0.026 & 503 & 1.682 & 0.064 & 325 \\
\hline G6 ............................ & 0.801 & 0.036 & 175 & 0.417 & 0.025 & 211 & 1.759 & 0.059 & 144 \\
\hline G8 & 0.852 & 0.039 & 186 & 0.445 & 0.035 & 246 & 1.866 & 0.088 & 191 \\
\hline K0 & 0.936 & 0.040 & 183 & 0.490 & 0.043 & 224 & 2.032 & 0.124 & 196 \\
\hline K1 & 1.001 & 0.036 & 102 & 0.528 & 0.034 & 117 & 2.176 & 0.095 & 100 \\
\hline K2 & 1.064 & 0.051 & 95 & 0.574 & 0.035 & 96 & 2.334 & 0.114 & 91 \\
\hline K3 & 1.150 & 0.073 & 98 & 0.605 & 0.041 & 91 & 2.483 & 0.126 & 83 \\
\hline K4 & 1.272 & 0.061 & 43 & 0.703 & 0.054 & 51 & 2.800 & 0.160 & 42 \\
\hline K5 & 1.420 & 0.093 & 22 & 0.781 & 0.043 & 21 & 3.237 & 0.222 & 21 \\
\hline
\end{tabular}

Chen et al. 1998). Finally, the FEPS sources studied by Bouwman et al. (2008) may have excess emission at $K$ band from a circumstellar disk. For these sources, we estimated the extinction using the observed $(R-I)_{\mathrm{C}}$ colors, the intrinsic colors compiled by Kenyon \& Hartmann (1995) and the reddening law from Taylor (1986). The adopted extinction values are listed in Table 5.

\section{B2. STELLAR EFFECTIVE TEMPERATURE, SURFACE GRAVITY, AND METALLICITY}

The stellar effective temperature, surface gravity, and metallicity are needed to fit the Kurucz model atmospheres (see Appendix C). This section summarizes the procedure to estimate these properties for the FEPS sample. The procedure depends on the stellar age, as solar-mass stars younger than $\sim 100 \mathrm{Myr}$ are contracting toward the main sequence and the surface gravity varies with age.

Stars older than 100 Myr in the FEPS sample were considered to be main-sequence stars and were assigned a surface gravity of $\log g=$ 4.50. Stellar effective temperatures were estimated from the $B-V$ and $V-K$ versus temperature relations derived by Houdashelt et al. (2000) after dereddening the observed photometry (see $\S$ B.1). If the temperature uncertainty derived from the photometry is larger than $130 \mathrm{~K}$, the temperature was instead computed from a temperature versus spectral type relation using the colors listed in Table 4, the Tycho-to-Johnson color transformations from Mamajek et al. $(2002,2006)$ and the Houdashelt et al. $(2000)$ color-temperature relations. A limit of $130 \mathrm{~K}$ was adopted since that is approximately the temperature uncertainty associated with \pm 2 spectral subclasses.

Solar-type stars younger than $100 \mathrm{Myr}$ will be contracting toward the main sequence and will generally have lower surface gravities. Derivation of the surface gravities and effective temperatures need to be solved jointly. First, the effective temperature was computed assuming the star is on the main sequence as described above. The surface gravity was then estimated from the D'Antona \& Mazzitelli (1997) pre-main-sequence evolutionary tracks using the derived temperature and assumed age from Hillenbrand et al. (in preparation). If the temperature was estimated from the spectral type, an iterative correction needs to be applied since the derived temperature depends on both the spectral type and surface gravity. For the estimated surface gravity, a new temperature was derived using the effective temperature as a function of spectral type and surface gravity relation in Gray (1992). With the new temperature, the surface gravity was rederived from the D'Antona \& Mazzitelli (1997) evolutionary tracks.

Finally, the metallicity was fixed to $[\mathrm{Fe} / \mathrm{H}]=0.13$ for the Hyades stars following the measurements from Paulson et al.2003). For all other stars, we assumed $[\mathrm{Fe} / \mathrm{H}]=0$. The adopted metallicity, effective temperature, and surface gravity for each star in the FEPS sample are listed in Table 5.

\section{APPENDIX C}

\section{MODEL PHOTOSPHERES}

In several FEPS studies, the observed Spitzer flux densities were compared to model photospheric flux densities to infer the presence of an infrared excess diagnostic of a circumstellar disk. Model flux densities were estimated from synthetic photosphere spectra computed by R. Kurucz ${ }^{18}$ from ATLAS 9 stellar atmospheric models with convective overshoot and a microturbulent velocity of $1 \mathrm{~km} \mathrm{~s}^{-1}$. In this section we describe the procedures used to normalize the synthetic spectra to observed photometry and to compute model flux densities.

\footnotetext{
${ }^{18}$ See http://kurucz.harvard.edu.
} 
TABLE 5

Adopted Stellar Properties

\begin{tabular}{|c|c|c|c|c|c|}
\hline Source & Spectral Type & $\begin{array}{c}A_{V} \\
\text { (mag) }\end{array}$ & $\begin{array}{c}{[\mathrm{Fe} / \mathrm{H}]} \\
(\mathrm{dex})\end{array}$ & $\begin{array}{c}\log g \\
\left(\log \mathrm{cm} \mathrm{s}^{-2}\right)\end{array}$ & $\begin{array}{l}T_{\text {eff }} \\
(\mathrm{K})\end{array}$ \\
\hline 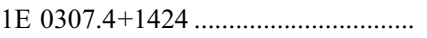 & G6 V & 0.03 & 0.00 & 4.54 & 5591 \\
\hline 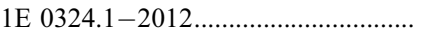 & G4 V & 0.04 & 0.00 & 4.54 & 5745 \\
\hline 1RXS J025216.9+361658 „................ & K2 IV & 0.95 & 0.00 & 4.63 & 4999 \\
\hline 1RXS J025751.8+115759 .................. & G7 V & 0.62 & 0.00 & 4.54 & 5552 \\
\hline 1RXS J030759.1+302032 ................. & G5 IV & 0.16 & 0.00 & 4.50 & 5676 \\
\hline 1RXS J031644.0+192259 „................. & G2 V & 0.10 & 0.00 & 4.47 & 5886 \\
\hline 1RXS J031907.4+393418 ................. & $\mathrm{K} 0 \mathrm{~V}$ & 0.19 & 0.00 & 4.61 & 5287 \\
\hline 1RXS J034423.3+281224_................ & G7 V & 0.05 & 0.00 & 4.54 & 5725 \\
\hline 1RXS J035028.0+163121 ................. & G5 IV & 0.34 & 0.00 & 4.54 & 5725 \\
\hline 1RXS J043243.2-152003 „................ & G4 V & 0.37 & 0.00 & 3.89 & 5760 \\
\hline 1RXS J051111.1+281353 „................ & $\mathrm{K} 0 \mathrm{~V}$ & 0.86 & 0.00 & 4.08 & 5325 \\
\hline 1RXS J053650.0+133756 .................... & $\mathrm{K} 0 \mathrm{~V}$ & 0.56 & 0.00 & 4.50 & 5323 \\
\hline 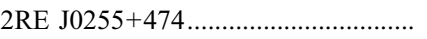 & $\mathrm{K} 5 \mathrm{Ve}$ & 0.00 & 0.00 & 4.71 & 4714 \\
\hline 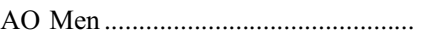 & $\mathrm{K} 3.5 \mathrm{~V} \mathrm{ke}$ & 0.00 & 0.00 & 4.41 & 4435 \\
\hline AP 93 & $\ldots$ & 0.31 & 0.00 & 4.68 & 4914 \\
\hline $\mathrm{B} 102 \ldots \ldots \ldots \ldots \ldots \ldots \ldots$ & $\ldots$ & 0.12 & 0.00 & 4.54 & 5708 \\
\hline 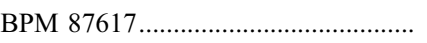 & $\mathrm{K} 5 \mathrm{Ve}$ & 0.00 & 0.00 & 4.71 & 4419 \\
\hline 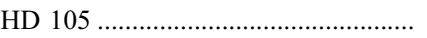 & G0 V & 0.00 & 0.00 & 4.48 & 5960 \\
\hline 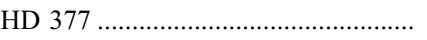 & $\mathrm{G} 2 \mathrm{~V}$ & 0.00 & 0.00 & 4.47 & 5851 \\
\hline 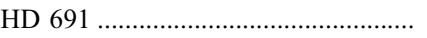 & $\mathrm{K} 0 \mathrm{~V}$ & 0.00 & 0.00 & 4.50 & 5441 \\
\hline 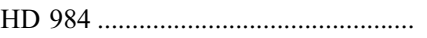 & F7 V & 0.00 & 0.00 & 4.40 & 6227 \\
\hline 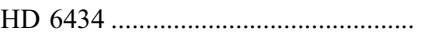 & $\mathrm{G} 2 / 3 \mathrm{~V}$ & 0.00 & 0.00 & 4.50 & 5804 \\
\hline 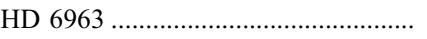 & G7 V & 0.00 & 0.00 & 4.50 & 5518 \\
\hline 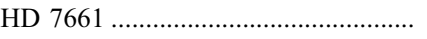 & $\mathrm{K} 0 \mathrm{~V}$ & 0.00 & 0.00 & 4.50 & 5399 \\
\hline 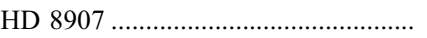 & F8 & 0.00 & 0.00 & 4.50 & 6250 \\
\hline 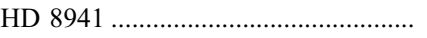 & F8 IV-V & 0.00 & 0.00 & 4.50 & 6234 \\
\hline 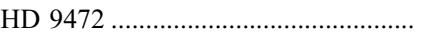 & G0 & 0.00 & 0.00 & 4.50 & 5686 \\
\hline 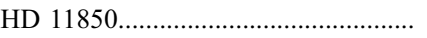 & G5 & 0.00 & 0.00 & 4.50 & 5597 \\
\hline 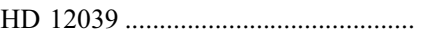 & $\mathrm{G} 3 / 5 \mathrm{~V}$ & 0.00 & 0.00 & 4.54 & 5688 \\
\hline 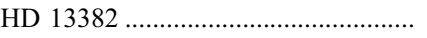 & G5 V & 0.00 & 0.00 & 4.50 & 5751 \\
\hline 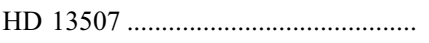 & G5 V & 0.00 & 0.00 & 4.50 & 5627 \\
\hline 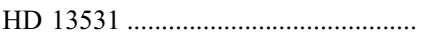 & G7 V & 0.00 & 0.00 & 4.50 & 5563 \\
\hline 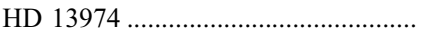 & G0 V & 0.00 & 0.00 & 4.50 & 5858 \\
\hline 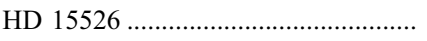 & G5/6 V & 0.13 & 0.00 & 4.54 & 5658 \\
\hline 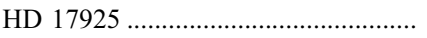 & K1 V & 0.00 & 0.00 & 4.68 & 5116 \\
\hline 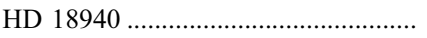 & G0 & 0.00 & 0.00 & 4.50 & 5760 \\
\hline 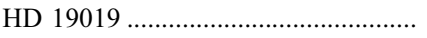 & F8 & 0.00 & 0.00 & 4.50 & 6051 \\
\hline 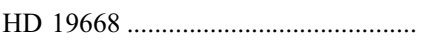 & $\mathrm{G} 8 / \mathrm{K} 0 \mathrm{~V}$ & 0.00 & 0.00 & 4.50 & 5414 \\
\hline 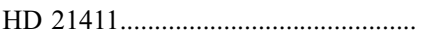 & G8 V & 0.00 & 0.00 & 4.50 & 5500 \\
\hline 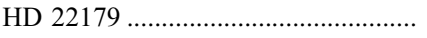 & G0 & 0.13 & 0.00 & 4.21 & 6000 \\
\hline 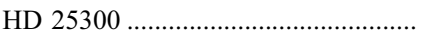 & K0 & 0.00 & 0.00 & 4.50 & 4329 \\
\hline 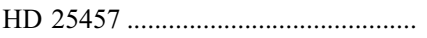 & F7 V & 0.00 & 0.00 & 4.50 & 6173 \\
\hline 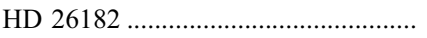 & G0 V & 0.34 & 0.00 & 4.47 & 6026 \\
\hline 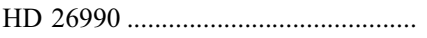 & G0(V) & 0.00 & 0.00 & 4.50 & 5651 \\
\hline 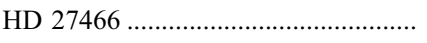 & G5 V & 0.00 & 0.00 & 4.50 & 5757 \\
\hline 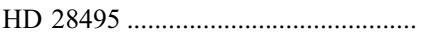 & G0 & 0.00 & 0.00 & 4.50 & 5217 \\
\hline 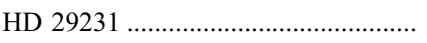 & G8 V & 0.00 & 0.00 & 4.50 & 5421 \\
\hline 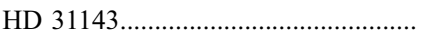 & $\mathrm{K} 0 \mathrm{~V}$ & 0.00 & 0.00 & 4.50 & 5313 \\
\hline 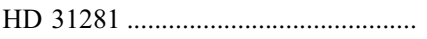 & G1(V) & 0.14 & 0.00 & 4.03 & 5927 \\
\hline 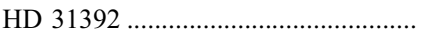 & $\mathrm{K} 0 \mathrm{~V}$ & 0.00 & 0.00 & 4.50 & 5360 \\
\hline 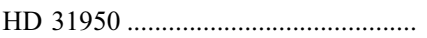 & $\ldots$ & 0.13 & 0.00 & 4.40 & 6108 \\
\hline HD 32850 & G9 V & 0.00 & 0.00 & 4.50 & 5208 \\
\hline 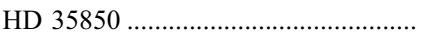 & F7/8 V & 0.00 & 0.00 & 4.04 & 6021 \\
\hline 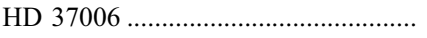 & G0 & 0.00 & 0.00 & 4.50 & 5503 \\
\hline 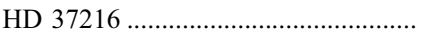 & G5 & 0.00 & 0.00 & 4.50 & 5387 \\
\hline 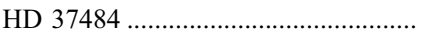 & F3 V & 0.00 & 0.00 & 4.27 & 6664 \\
\hline 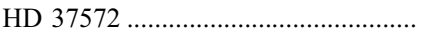 & $\mathrm{K} 0 \mathrm{~V}$ & 0.00 & 0.00 & 4.67 & 5091 \\
\hline 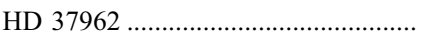 & G5 V & 0.00 & 0.00 & 4.50 & 5718 \\
\hline 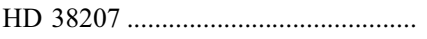 & F2 V & 0.05 & 0.00 & 4.50 & 6762 \\
\hline 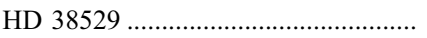 & G8 III/IV & 0.00 & 0.00 & 4.50 & 5361 \\
\hline 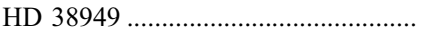 & G1 V & 0.00 & 0.00 & 4.50 & 6028 \\
\hline 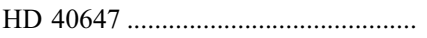 & G5 & 0.00 & 0.00 & 4.50 & 5268 \\
\hline 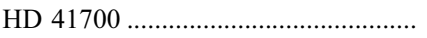 & $\mathrm{F} 8 / \mathrm{G} 0 \mathrm{~V}$ & 0.00 & 0.00 & 4.50 & 6138 \\
\hline 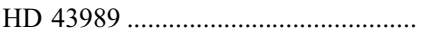 & G0 V & 0.00 & 0.00 & 4.47 & 5958 \\
\hline 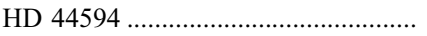 & G3 V & 0.00 & 0.00 & 4.50 & 5784 \\
\hline
\end{tabular}


TABLE 5-Continued

\begin{tabular}{|c|c|c|c|c|c|}
\hline Source & Spectral Type & $\begin{array}{c}A_{V} \\
(\mathrm{mag})\end{array}$ & $\begin{array}{c}{[\mathrm{Fe} / \mathrm{H}]} \\
(\mathrm{dex})\end{array}$ & $\begin{array}{c}\log g \\
\left(\log \mathrm{cm} \mathrm{s}^{-2}\right)\end{array}$ & $\begin{array}{l}T_{\text {eff }} \\
(\mathrm{K})\end{array}$ \\
\hline HD 45270 & G1 V & 0.00 & 0.00 & 4.47 & 5885 \\
\hline HD 47875 & G3 V & 0.34 & 0.00 & 4.47 & 5825 \\
\hline HD 60737 & G0 & 0.00 & 0.00 & 4.50 & 5895 \\
\hline HD 61005 & G8 Vk & 0.00 & 0.00 & 4.50 & 5463 \\
\hline HD $61994 \ldots \ldots$ & G6 V & 0.00 & 0.00 & 4.50 & 5538 \\
\hline HD 64324 & G0 & 0.00 & 0.00 & 4.50 & 5737 \\
\hline HD $66751 \ldots \ldots \ldots$ & F8 V & 0.00 & 0.00 & 4.50 & 5854 \\
\hline HD 69076 & $\mathrm{~K} 0 \mathrm{~V}$ & 0.00 & 0.00 & 4.50 & 5405 \\
\hline HD 70516 & G0 & 0.00 & 0.00 & 4.54 & 5735 \\
\hline HD 70573 & $\mathrm{G} 1 / 2 \mathrm{~V}$ & 0.04 & 0.00 & 4.47 & 5896 \\
\hline HD 71974 & G5 & 0.00 & 0.00 & 4.50 & 5436 \\
\hline HD 72687 & G5 V & 0.00 & 0.00 & 4.50 & 5738 \\
\hline HD 72905 & G1.5 VB & 0.00 & 0.00 & 4.50 & 5834 \\
\hline HD 73668 & G1 V & 0.00 & 0.00 & 4.50 & 5876 \\
\hline HD $75302 \ldots \ldots \ldots$ & G5 V & 0.00 & 0.00 & 4.50 & 5674 \\
\hline 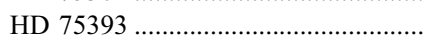 & F7 V & 0.00 & 0.00 & 4.50 & 6055 \\
\hline HD $76218 \ldots \ldots \ldots$ & G9-V & 0.00 & 0.00 & 4.50 & 5359 \\
\hline HD 77407 & G0(V) & 0.00 & 0.00 & 4.53 & 5734 \\
\hline HD 80606 & G5 & 0.12 & 0.00 & 4.50 & 5668 \\
\hline HD $85301 \ldots \ldots \ldots \ldots$ & G5 & 0.00 & 0.00 & 4.50 & 5611 \\
\hline HD $86356 \ldots \ldots \ldots$ & G6/K0 & 0.40 & 0.00 & 4.54 & 5485 \\
\hline HD $88201 \ldots \ldots \ldots$ & G0 V & 0.00 & 0.00 & 4.50 & 6079 \\
\hline HD 88742 & G0 V & 0.00 & 0.00 & 4.50 & 5954 \\
\hline HD 90712 & $\mathrm{G} 2 / 3 \mathrm{~V}$ & 0.00 & 0.00 & 4.50 & 5873 \\
\hline HD 90905 & G1 V & 0.00 & 0.00 & 4.50 & 6028 \\
\hline HD 91782 & G0 & 0.00 & 0.00 & 4.50 & 6107 \\
\hline HD 91962 & G1 V & 0.00 & 0.00 & 4.50 & 5623 \\
\hline HD 92788 & G6 V & 0.00 & 0.00 & 4.50 & 5681 \\
\hline HD 92855 & F9 V & 0.00 & 0.00 & 4.50 & 5981 \\
\hline HD 95188 & G8 V & 0.00 & 0.00 & 4.50 & 5400 \\
\hline HD 98553 & $\mathrm{G} 2 / 3 \mathrm{~V}$ & 0.00 & 0.00 & 4.50 & 5917 \\
\hline HD $100167 \ldots \ldots \ldots$ & F8 & 0.00 & 0.00 & 4.50 & 5779 \\
\hline HD 101472 & F7 V & 0.00 & 0.00 & 4.50 & 6184 \\
\hline HD 101959 & G0 V & 0.00 & 0.00 & 4.50 & 6048 \\
\hline 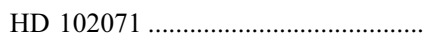 & $\mathrm{K} 0 \mathrm{~V}$ & 0.00 & 0.00 & 4.50 & 5297 \\
\hline HD $103432 \ldots \ldots \ldots$ & G6 V & 0.00 & 0.00 & 4.50 & 5597 \\
\hline HD $104467 \ldots \ldots \ldots$ & G5 III/IV & 0.13 & 0.00 & 4.06 & 5681 \\
\hline HD $104576 \ldots \ldots \ldots \ldots$ & G3 V & 0.00 & 0.00 & 4.50 & 5436 \\
\hline HD $104860 \ldots \ldots \ldots \ldots$ & F8 & 0.00 & 0.00 & 4.47 & 5951 \\
\hline HD $105631 \ldots \ldots \ldots$ & G9 V & 0.00 & 0.00 & 4.50 & 5343 \\
\hline HD 106156 & G8 V & 0.00 & 0.00 & 4.50 & 5403 \\
\hline HD 106252 & G0 V & 0.00 & 0.00 & 4.50 & 5876 \\
\hline HD $106772 \ldots \ldots$ & G2 III/IV & 0.00 & 0.00 & 4.50 & 4928 \\
\hline HD 107146 & G2 V & 0.00 & 0.00 & 4.50 & 5841 \\
\hline HD 107441 & G1.5 IV & 0.28 & 0.00 & 4.21 & 5926 \\
\hline HD 108799 & $\mathrm{G} 1 / 2 \mathrm{~V}$ & 0.00 & 0.00 & 4.50 & 5815 \\
\hline HD 108944 & F9 V & 0.00 & 0.00 & 4.50 & 6138 \\
\hline HD $111170 \ldots \ldots \ldots \ldots$ & $\mathrm{G} 8 / \mathrm{K} 0 \mathrm{~V}$ & 0.41 & 0.00 & 4.35 & 5384 \\
\hline 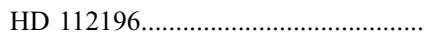 & F8 V & 0.00 & 0.00 & 4.47 & 5950 \\
\hline 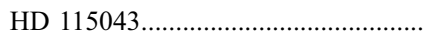 & G1 V & 0.00 & 0.00 & 4.50 & 5846 \\
\hline 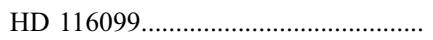 & $\mathrm{G} 0 / 3$ & 0.07 & 0.00 & 4.21 & 5899 \\
\hline 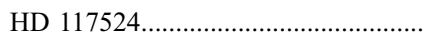 & G2.5 IV & 0.62 & 0.00 & 4.21 & 5902 \\
\hline 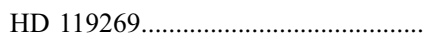 & $\mathrm{G} 3 / 5 \mathrm{~V}$ & 0.14 & 0.00 & 4.21 & 5732 \\
\hline HD 120812 & F8/G0 V & 0.37 & 0.00 & 4.12 & 6081 \\
\hline HD $121320 \ldots \ldots \ldots$ & G5 V & 0.00 & 0.00 & 4.50 & 5637 \\
\hline HD $121504 \ldots \ldots \ldots \ldots$ & G2 V & 0.00 & 0.00 & 4.50 & 5967 \\
\hline 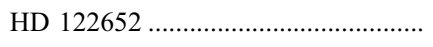 & F8 & 0.00 & 0.00 & 4.50 & 6163 \\
\hline HD 126670 & G6/8 III/IV & 0.29 & 0.00 & 4.26 & 5528 \\
\hline 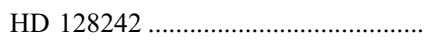 & G3 V & 0.36 & 0.00 & 4.26 & 5823 \\
\hline HD 129333 & G5 V & 0.00 & 0.00 & 4.54 & 5653 \\
\hline 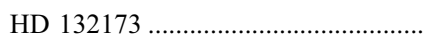 & G0 V & 0.00 & 0.00 & 4.50 & 5980 \\
\hline 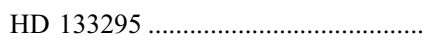 & G0/1 V & 0.00 & 0.00 & 4.50 & 6003 \\
\hline HD 133938 & G6/8 III/IV & 0.31 & 0.00 & 4.26 & 5566 \\
\hline HD 134319 & G5(V) & 0.00 & 0.00 & 4.54 & 5660 \\
\hline 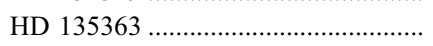 & G5(V) & 0.00 & 0.00 & 4.66 & 4728 \\
\hline HD 136923 & G9 V & 0.00 & 0.00 & 4.50 & 5343 \\
\hline
\end{tabular}


TABLE 5-Continued

\begin{tabular}{|c|c|c|c|c|c|}
\hline Source & Spectral Type & $\begin{array}{c}A_{V} \\
(\mathrm{mag})\end{array}$ & $\begin{array}{c}{[\mathrm{Fe} / \mathrm{H}]} \\
(\mathrm{dex})\end{array}$ & $\begin{array}{c}\log g \\
\left(\log \mathrm{cm} \mathrm{s}^{-2}\right)\end{array}$ & $\begin{array}{l}T_{\text {eff }} \\
(\mathrm{K})\end{array}$ \\
\hline HD 138004 & G2 III & 0.00 & 0.00 & 4.50 & 5715 \\
\hline HD 139498 & G8(V) & 0.26 & 0.00 & 4.26 & 5473 \\
\hline HD 139813 & G5 & 0.00 & 0.00 & 4.50 & 5380 \\
\hline HD 140374 & G8 V & 0.09 & 0.00 & 4.26 & 5444 \\
\hline HD 141521 & G8 V & 0.06 & 0.00 & 4.26 & 5450 \\
\hline 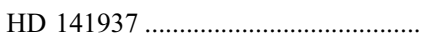 & $\mathrm{G} 2 / 3 \mathrm{~V}$ & 0.00 & 0.00 & 4.50 & 5844 \\
\hline HD 141943 & $\mathrm{G} 0 / 2 \mathrm{~V}$ & 0.14 & 0.00 & 4.23 & 5943 \\
\hline 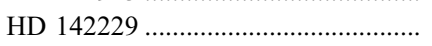 & G5 V & 0.00 & 0.00 & 4.50 & 5861 \\
\hline HD 142361 & G3 V & 0.46 & 0.00 & 3.70 & 5846 \\
\hline HD 143006 & G6/8 & 0.58 & 0.00 & 3.70 & 5817 \\
\hline HD 143358 & $\mathrm{G} 1 / 2 \mathrm{~V}$ & 0.01 & 0.00 & 4.12 & 5899 \\
\hline HD 145229 & G0 & 0.00 & 0.00 & 4.50 & 5895 \\
\hline HD $146516 \ldots \ldots \ldots \ldots$ & G0 IV & 0.83 & 0.00 & 3.70 & 6024 \\
\hline HD 150554 & F8 & 0.00 & 0.00 & 4.50 & 5975 \\
\hline HD 150706 & G3(V) & 0.00 & 0.00 & 4.50 & 5885 \\
\hline HD 151798 & G3 V & 0.00 & 0.00 & 4.47 & 5878 \\
\hline HD 152555 & $\mathrm{~F} 8 / \mathrm{G} 0 \mathrm{~V}$ & 0.00 & 0.00 & 4.50 & 5891 \\
\hline HD 153458 & G5 V & 0.00 & 0.00 & 4.50 & 5768 \\
\hline 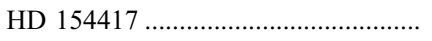 & F9 V & 0.00 & 0.00 & 4.50 & 6014 \\
\hline HD 157664 & G0 & 0.05 & 0.00 & 4.50 & 6251 \\
\hline HD 159222 & G1 V & 0.00 & 0.00 & 4.50 & 5774 \\
\hline 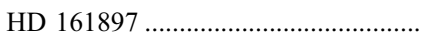 & K0 & 0.00 & 0.00 & 4.50 & 5558 \\
\hline HD 167389 & $\mathrm{~F} 8(\mathrm{~V})$ & 0.00 & 0.00 & 4.50 & 5846 \\
\hline HD 170778 & G5 & 0.00 & 0.00 & 4.50 & 5893 \\
\hline HD 172649 & F5 & 0.00 & 0.00 & 4.50 & 6172 \\
\hline HD 174656 & G6 IV & 0.83 & 0.00 & 3.95 & 5628 \\
\hline HD 179949 & F8 V & 0.00 & 0.00 & 4.50 & 6116 \\
\hline HD 183216 & $\mathrm{G} 2 \mathrm{~V}$ & 0.00 & 0.00 & 4.50 & 6002 \\
\hline 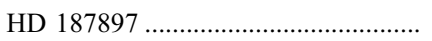 & G5 & 0.00 & 0.00 & 4.50 & 5883 \\
\hline HD 190228 & G5 IV & 0.00 & 0.00 & 4.50 & 5246 \\
\hline HD 191089 & F5 V & 0.00 & 0.00 & 4.50 & 6450 \\
\hline 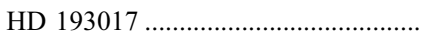 & F6 V & 0.00 & 0.00 & 4.50 & 6121 \\
\hline HD 195034 & G5 & 0.00 & 0.00 & 4.50 & 5800 \\
\hline HD 199019 & G5 & 0.00 & 0.00 & 4.50 & 5485 \\
\hline 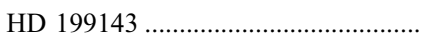 & F8 V & 0.00 & 0.00 & 4.23 & 5895 \\
\hline HD 199598 & G0 V & 0.00 & 0.00 & 4.50 & 5882 \\
\hline HD 200746 & G5 & 0.00 & 0.00 & 4.50 & 5701 \\
\hline HD 201219 & G5 & 0.00 & 0.00 & 4.50 & 5610 \\
\hline HD $201989 \ldots \ldots \ldots \ldots$ & $\mathrm{G} 3 / 5 \mathrm{~V}$ & 0.00 & 0.00 & 4.50 & 5636 \\
\hline HD 202108 & G3 V & 0.00 & 0.00 & 4.50 & 5722 \\
\hline HD $202917 \ldots \ldots \ldots$ & G5 V & 0.00 & 0.00 & 4.50 & 5555 \\
\hline HD 203030 & G8 V & 0.00 & 0.00 & 4.50 & 5416 \\
\hline HD $204277 \ldots \ldots \ldots$ & F8 V & 0.00 & 0.00 & 4.50 & 6189 \\
\hline HD $205905 \ldots \ldots \ldots \ldots$ & $\mathrm{G} 2 \mathrm{~V}$ & 0.00 & 0.00 & 4.50 & 5925 \\
\hline HD 206374 & $\mathrm{G} 6.5 \mathrm{~V}$ & 0.00 & 0.00 & 4.50 & 5577 \\
\hline HD 209253 & $\mathrm{~F} 6 / 7 \mathrm{~V}$ & 0.00 & 0.00 & 4.50 & 6211 \\
\hline HD 209393 & G5 & 0.00 & 0.00 & 4.50 & 5632 \\
\hline HD 209779 & G2 V & 0.00 & 0.00 & 4.50 & 5575 \\
\hline HD $212291 \ldots \ldots \ldots$ & G5 & 0.00 & 0.00 & 4.50 & 5622 \\
\hline HD $216275 \ldots \ldots \ldots$ & G0 & 0.00 & 0.00 & 4.50 & 5956 \\
\hline HD $216803 \ldots \ldots \ldots$ & K4 VP & 0.00 & 0.00 & 4.50 & 4624 \\
\hline HD $217343 \ldots \ldots \ldots \ldots \ldots$ & $\mathrm{G} 3 \mathrm{~V}$ & 0.00 & 0.00 & 4.47 & 5772 \\
\hline 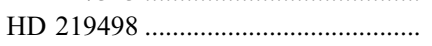 & G5 & 0.07 & 0.00 & 4.50 & 5666 \\
\hline HD 224873 & K0 & 0.00 & 0.00 & 4.50 & 5181 \\
\hline HD 245567 ........................................ & G0 V & 0.61 & 0.00 & 3.91 & 6042 \\
\hline HD $279788 \ldots \ldots \ldots$ & G5 V & 0.63 & 0.00 & 3.80 & 5713 \\
\hline HD $281691 \ldots \ldots \ldots \ldots$ & $\mathrm{K} 1(\mathrm{~V})$ & 0.16 & 0.00 & 4.38 & 5127 \\
\hline HD $282346 \ldots \ldots \ldots$ & G8 V & 0.55 & 0.00 & 4.50 & 5475 \\
\hline HD $284135 \ldots \ldots \ldots \ldots$ & G3(V) & 0.08 & 0.00 & 4.03 & 5799 \\
\hline HD $284266 \ldots \ldots \ldots \ldots$ & $\mathrm{K} 0(\mathrm{~V})$ & 0.08 & 0.00 & 4.34 & 5426 \\
\hline HD $285281 \ldots \ldots \ldots$ & $\mathrm{K} 1$ & 0.69 & 0.00 & 4.32 & 5135 \\
\hline HD $285372 \ldots \ldots \ldots \ldots$ & $\mathrm{K} 3(\mathrm{~V})$ & 0.72 & 0.00 & 4.21 & 5039 \\
\hline HD $285751 \ldots \ldots$ & $\mathrm{K} 2(\mathrm{~V})$ & 0.47 & 0.00 & 4.21 & 5017 \\
\hline HD 285840 & $\mathrm{~K} 1(\mathrm{~V})$ & 0.00 & 0.00 & 4.61 & 5165 \\
\hline HD 286179 & G3(V) & 0.38 & 0.00 & 4.21 & 5843 \\
\hline HD 286264 & K2 IV & 1.11 & 0.00 & 4.49 & 4991 \\
\hline
\end{tabular}


TABLE 5-Continued

\begin{tabular}{|c|c|c|c|c|c|}
\hline Source & Spectral Type & $\begin{array}{c}A_{V} \\
\text { (mag) }\end{array}$ & $\begin{array}{c}{[\mathrm{Fe} / \mathrm{H}]} \\
(\mathrm{dex})\end{array}$ & $\begin{array}{c}\log g \\
\left(\log \mathrm{cm} \mathrm{s}^{-2}\right)\end{array}$ & $\begin{array}{l}T_{\text {eff }} \\
(\mathrm{K})\end{array}$ \\
\hline HE 350 & $\ldots$ & 0.31 & 0.00 & 4.47 & 5922 \\
\hline HE 373 & $\ldots$ & 0.31 & 0.00 & 4.60 & 5399 \\
\hline HE 389 & $\ldots$ & 0.31 & 0.00 & 4.47 & 6043 \\
\hline HE 622 & $\ldots$ & 0.31 & 0.00 & 4.54 & 5497 \\
\hline HE 696 & $\ldots$ & 0.31 & 0.00 & 4.54 & 5756 \\
\hline HE 699 & $\ldots$ & 0.31 & 0.00 & 4.54 & 5634 \\
\hline HE 750 & F5 & 0.31 & 0.00 & 4.33 & 6421 \\
\hline HE $767 \ldots \ldots \ldots \ldots$ & $\ldots$ & 0.31 & 0.00 & 4.40 & 6219 \\
\hline HE 848 & F9 V & 0.31 & 0.00 & 4.40 & 6309 \\
\hline HE 935 & F9.5 V & 0.31 & 0.00 & 4.40 & 6115 \\
\hline HE 1101 & $\ldots$ & 0.31 & 0.00 & 4.47 & 5823 \\
\hline HE 1234 & G2 & 0.31 & 0.00 & 4.54 & 5738 \\
\hline HII $120 \ldots \ldots \ldots$ & G6 V & 0.12 & 0.00 & 4.50 & 5707 \\
\hline 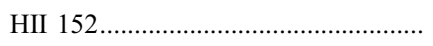 & G5 V & 0.12 & 0.00 & 4.50 & 5823 \\
\hline HII 173 & $\ldots$ & 0.12 & 0.00 & 4.50 & 5266 \\
\hline HII 174 & $\ldots$ & 0.12 & 0.00 & 4.50 & 4998 \\
\hline HII $250 \ldots \ldots \ldots \ldots$ & $\ldots$ & 0.12 & 0.00 & 4.50 & 5767 \\
\hline HII 314 & $\ldots$ & 0.12 & 0.00 & 4.50 & 5788 \\
\hline HII 514 & $\ldots$ & 0.12 & 0.00 & 4.50 & 5727 \\
\hline 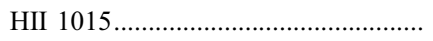 & $\ldots$ & 0.12 & 0.00 & 4.50 & 5904 \\
\hline HII $1101 \ldots \ldots \ldots \ldots$ & G0 V & 0.12 & 0.00 & 4.50 & 5988 \\
\hline 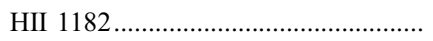 & F8 & 0.12 & 0.00 & 4.50 & 5845 \\
\hline HII 1200 & F6 V & 0.12 & 0.00 & 4.50 & 6217 \\
\hline HII 1776 & G5 & 0.12 & 0.00 & 4.50 & 5622 \\
\hline HII $2147 \ldots \ldots \ldots \ldots$ & G7 IV & 0.12 & 0.00 & 4.50 & 5089 \\
\hline 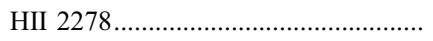 & $\ldots$ & 0.12 & 0.00 & 4.50 & 5213 \\
\hline 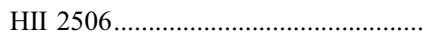 & F9 & 0.12 & 0.00 & 4.50 & 6082 \\
\hline 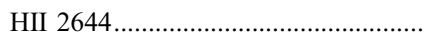 & $\ldots$ & 0.12 & 0.00 & 4.50 & 5614 \\
\hline 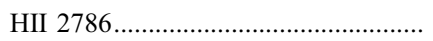 & $\ldots$ & 0.12 & 0.00 & 4.50 & 6068 \\
\hline HII $2881 \ldots \ldots \ldots \ldots$ & $\mathrm{K} 2$ & 0.12 & 0.00 & 4.50 & 4844 \\
\hline 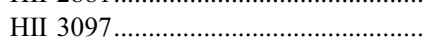 & $\ldots$ & 0.12 & 0.00 & 4.50 & 5585 \\
\hline 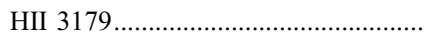 & $\ldots$ & 0.12 & 0.00 & 4.50 & 6137 \\
\hline HIP 6276 & G0 & 0.00 & 0.00 & 4.50 & 5352 \\
\hline HIP $42491 \ldots \ldots$ & G5 & 0.00 & 0.00 & 4.50 & 5246 \\
\hline 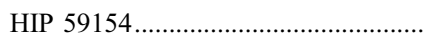 & $\mathrm{K} 2$ & 0.00 & 0.00 & 4.50 & 4390 \\
\hline HIP $76477 \ldots \ldots \ldots$ & G9 & 0.43 & 0.00 & 4.38 & 5389 \\
\hline 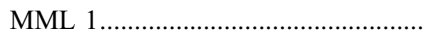 & $\mathrm{K} 1+\mathrm{IV}$ & 0.56 & 0.00 & 4.44 & 5150 \\
\hline MML 8 & $\mathrm{K} 0+\mathrm{IV}$ & 0.48 & 0.00 & 4.35 & 5278 \\
\hline MML 9 & G9 IV & 0.34 & 0.00 & 4.35 & 5360 \\
\hline 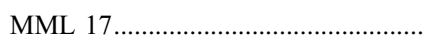 & G0 IV & 0.28 & 0.00 & 4.21 & 6002 \\
\hline 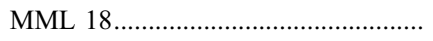 & $\mathrm{K} 0+\mathrm{IV}$ & 0.61 & 0.00 & 4.35 & 5290 \\
\hline 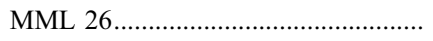 & G5 IV & 0.33 & 0.00 & 4.21 & 5711 \\
\hline 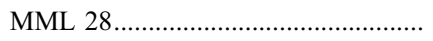 & $\mathrm{K} 2-\mathrm{IV}$ & 0.06 & 0.00 & 4.44 & 4970 \\
\hline MML $32 \ldots \ldots \ldots \ldots$ & G1 IV & 0.61 & 0.00 & 4.21 & 5991 \\
\hline 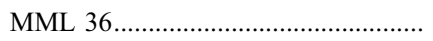 & K0 IV & 0.27 & 0.00 & 4.38 & 5271 \\
\hline 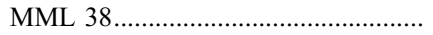 & G8 IVe & 0.45 & 0.00 & 4.26 & 5487 \\
\hline 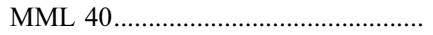 & G9 IV & 0.45 & 0.00 & 4.38 & 5382 \\
\hline 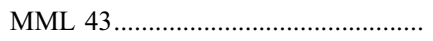 & G7 IV & 0.36 & 0.00 & 4.26 & 5525 \\
\hline 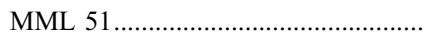 & K1 IVe & 0.67 & 0.00 & 4.38 & 5142 \\
\hline 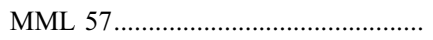 & G1.5 IV & 0.21 & 0.00 & 4.12 & 5913 \\
\hline PDS 66 & K1 IVe & 0.57 & 0.00 & 4.35 & 5256 \\
\hline [PZ99] J155847.8-175800 .................. & K3 & 1.41 & 0.00 & 4.22 & 4889 \\
\hline [PZ99] J160814.7-190833 .................. & $\mathrm{K} 2$ & 1.06 & 0.00 & 4.19 & 4997 \\
\hline [PZ99] J161318.6-221248 ................... & G9 & 1.22 & 0.00 & 4.06 & 5396 \\
\hline 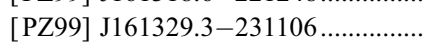 & $\mathrm{K} 1$ & 1.25 & 0.00 & 4.19 & 5154 \\
\hline [PZ99] J161402.1-230101 ….............. & G4 & 1.35 & 0.00 & 4.06 & 5783 \\
\hline [PZ99] J161411.0-230536 ................ & K0 & 1.15 & 0.00 & 4.16 & 5312 \\
\hline [PZ99] J161459.2-275023 .................. & G5 & 0.95 & 0.00 & 4.06 & 5724 \\
\hline [PZ99] J161618.0-233947 ................. & G7 & 0.83 & 0.00 & 4.06 & 5553 \\
\hline 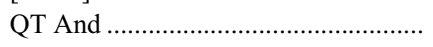 & $\mathrm{K} 7 \mathrm{Ve}$ & 0.00 & 0.00 & 4.62 & 4692 \\
\hline 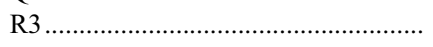 & $\ldots$ & 0.12 & 0.00 & 4.61 & 5214 \\
\hline R45 & $\ldots$ & 0.12 & 0.00 & 4.47 & 5947 \\
\hline R83 & $\ldots$ & 0.12 & 0.00 & 4.47 & 5796 \\
\hline 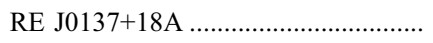 & $\mathrm{K} 3 \mathrm{Ve}$ & 1.47 & 0.00 & 4.38 & 5001 \\
\hline 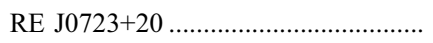 & K5 VE & 0.00 & 0.00 & 4.50 & 4408 \\
\hline 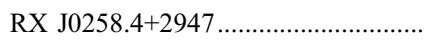 & K0 IV & 0.41 & 0.00 & 4.61 & 5274 \\
\hline
\end{tabular}


TABLE 5-Continued

\begin{tabular}{|c|c|c|c|c|c|}
\hline Source & Spectral Type & $\begin{array}{c}A_{V} \\
\text { (mag) }\end{array}$ & $\begin{array}{c}{[\mathrm{Fe} / \mathrm{H}]} \\
(\mathrm{dex})\end{array}$ & $\begin{array}{c}\log g \\
\left(\log \mathrm{cm} \mathrm{s}^{-2}\right)\end{array}$ & $\begin{array}{l}T_{\text {eff }} \\
(\mathrm{K})\end{array}$ \\
\hline RX J0329.1+0118 ……............................... & G0(IV) & 0.04 & 0.00 & 4.50 & 6150 \\
\hline 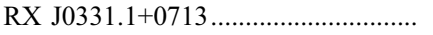 & $\mathrm{K} 4(\mathrm{~V}) / \mathrm{E}$ & 0.08 & 0.00 & 4.01 & 4777 \\
\hline RX J0354.4+0535 & G2(V) & 0.01 & 0.00 & 4.47 & 5846 \\
\hline RX J0357.3+1258 & G0 & 0.59 & 0.00 & 4.47 & 6037 \\
\hline RX J0434.3+0226 & $\mathrm{K} 4 \mathrm{e}$ & 0.37 & 0.00 & 4.63 & 4666 \\
\hline 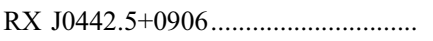 & G5(V) & 0.45 & 0.00 & 4.54 & 5700 \\
\hline RX J0849.2-7735 & $\mathrm{K} 2$ & 0.00 & 0.00 & 4.50 & 4382 \\
\hline RX J0850.1-7554 & G5 & 0.31 & 0.00 & 4.54 & 5713 \\
\hline 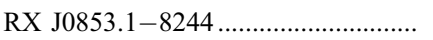 & $\mathrm{K} 0(\mathrm{~V})$ & 0.65 & 0.00 & 4.50 & 5317 \\
\hline RX J0917.2-7744 & G2 & 0.32 & 0.00 & 4.47 & 5888 \\
\hline RX J1111.7-7620 & $\mathrm{K} 1$ & 1.50 & 0.00 & 4.21 & 4653 \\
\hline 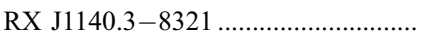 & $\mathrm{K} 2$ & 0.67 & 0.00 & 4.67 & 4970 \\
\hline 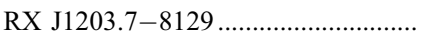 & $\mathrm{K} 1$ & 0.46 & 0.00 & 4.50 & 5229 \\
\hline RX J1209.8-7344 & G9 & 1.46 & 0.00 & 4.50 & 5426 \\
\hline 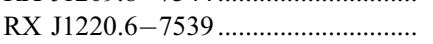 & $\mathrm{K} 2$ & 0.54 & 0.00 & 4.67 & 4997 \\
\hline 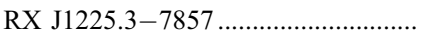 & G5 & 0.50 & 0.00 & 4.50 & 5732 \\
\hline 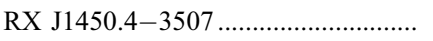 & K1(IV) & 0.52 & 0.00 & 4.38 & 5158 \\
\hline 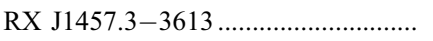 & G6 IV & 0.32 & 0.00 & 4.26 & 5590 \\
\hline 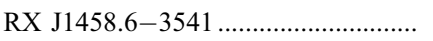 & K3(IV) & 0.56 & 0.00 & 4.44 & 4852 \\
\hline 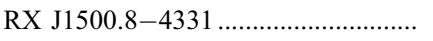 & K1(IV) & 0.28 & 0.00 & 4.38 & 5158 \\
\hline RX J1507.2-3505 & K0 & 0.24 & 0.00 & 4.38 & 5263 \\
\hline 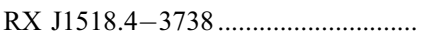 & $\mathrm{K} 1$ & 0.37 & 0.00 & 4.38 & 5166 \\
\hline RX J1531.3-3329 & K0 & 0.13 & 0.00 & 4.50 & 5304 \\
\hline RX J1541.1-2656 & G7 & 0.66 & 0.00 & 4.06 & 5542 \\
\hline 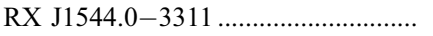 & $\mathrm{K} 1$ & 0.36 & 0.00 & 4.38 & 5150 \\
\hline 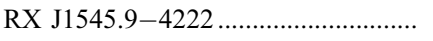 & $\mathrm{K} 1$ & 0.56 & 0.00 & 4.38 & 5143 \\
\hline RX J1600.6-2159 & G9 & 0.80 & 0.00 & 4.06 & 5375 \\
\hline 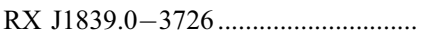 & $\mathrm{K} 1$ & 0.41 & 0.00 & 4.45 & 5119 \\
\hline 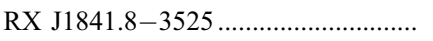 & G7 & 0.16 & 0.00 & 4.34 & 5534 \\
\hline 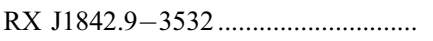 & $\mathrm{K} 2$ & 1.06 & 0.00 & 4.27 & 4645 \\
\hline 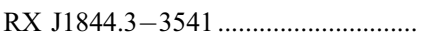 & K5 & 0.15 & 0.00 & 3.91 & 4648 \\
\hline 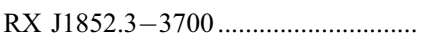 & $\mathrm{K} 3$ & 0.97 & 0.00 & 4.31 & 4854 \\
\hline 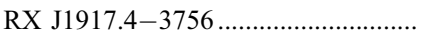 & $\mathrm{K} 2$ & 0.44 & 0.00 & 4.21 & 5033 \\
\hline RX J2313.0+2345 & F8 & 0.16 & 0.00 & 4.01 & 6105 \\
\hline SAO 150676 & $\mathrm{G} 2 \mathrm{~V}$ & 0.02 & 0.00 & 4.47 & 5862 \\
\hline SAO 178272 & $\mathrm{~K} 2 \mathrm{~V}$ & 0.42 & 0.00 & 4.50 & 4963 \\
\hline 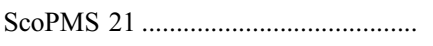 & K1 IV & 1.58 & 0.00 & 3.70 & 5806 \\
\hline 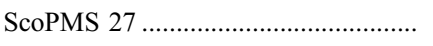 & K2 IV & 1.10 & 0.00 & 4.19 & 5066 \\
\hline 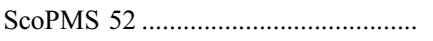 & K0 IV & 1.54 & 0.00 & 4.06 & 5372 \\
\hline ScoPMS 214 & K0 IV & 1.75 & 0.00 & 4.16 & 5331 \\
\hline 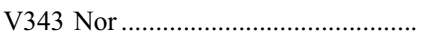 & $\mathrm{K} 0 \mathrm{~V}$ & 0.00 & 0.00 & 4.38 & 5002 \\
\hline 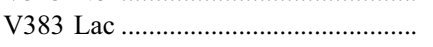 & K0 IV & 0.00 & 0.00 & 4.67 & 5121 \\
\hline 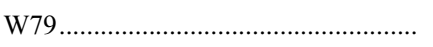 & $\ldots$ & 0.12 & 0.00 & 4.61 & 5415 \\
\hline vB 1 & F8 & 0.00 & 0.13 & 4.50 & 5983 \\
\hline 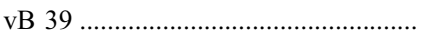 & G4 V & 0.00 & 0.13 & 4.50 & 5633 \\
\hline 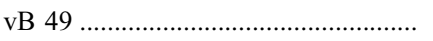 & G0 V & 0.00 & 0.13 & 4.50 & 5927 \\
\hline vB 52 & $\mathrm{G} 2 \mathrm{~V}$ & 0.00 & 0.13 & 4.50 & 5841 \\
\hline 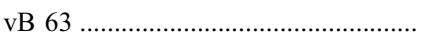 & G1 V & 0.00 & 0.13 & 4.50 & 5694 \\
\hline vB 64 & $\mathrm{G} 2+$ & 0.00 & 0.13 & 4.50 & 5733 \\
\hline 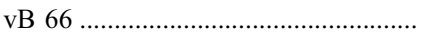 & F8 & 0.00 & 0.13 & 4.50 & 6058 \\
\hline vB 73 . & $\mathrm{G} 2 \mathrm{~V}$ & 0.00 & 0.13 & 4.50 & 5926 \\
\hline 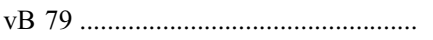 & $\mathrm{K} 0 \mathrm{~V}$ & 0.00 & 0.13 & 4.50 & 5273 \\
\hline vB 88 & F9 V & 0.00 & 0.13 & 4.50 & 6107 \\
\hline vB 91 & $\ldots$ & 0.00 & 0.13 & 4.50 & 5021 \\
\hline vB 92 & $\ldots$ & 0.00 & 0.13 & 4.50 & 5514 \\
\hline vB 93 & $\mathrm{~K} 2 \mathrm{~V}$ & 0.00 & 0.13 & 4.50 & 5112 \\
\hline vB 96 & G5 & 0.00 & 0.13 & 4.50 & 5165 \\
\hline 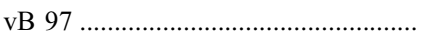 & $\mathrm{F} 8: \mathrm{V}:$ & 0.00 & 0.13 & 4.50 & 5873 \\
\hline vB 99 & K0 & 0.00 & 0.13 & 4.50 & 5156 \\
\hline vB 106 & G5 & 0.00 & 0.13 & 4.50 & 5782 \\
\hline vB 142 & G5 & 0.00 & 0.13 & 4.50 & 5652 \\
\hline vB 143 & F8 & 0.00 & 0.13 & 4.50 & 6228 \\
\hline vB 176 & $\mathrm{~K} 2 \mathrm{~V}$ & 0.00 & 0.13 & 4.50 & 4942 \\
\hline vB 180 & $\mathrm{~K} 1 \mathrm{~V}$ & 0.00 & 0.13 & 4.50 & 5216 \\
\hline vB 183 & K2 V & 0.00 & 0.13 & 4.50 & 5037 \\
\hline
\end{tabular}




\section{C1. OPTICAL AND NEAR-INFRARED PHOTOMETRY}

Synthetic spectra were normalized to published optical and near-infrared broadband photometry. Photometric catalogs incorporated for this study include Tycho-2 (Høg et al. 2000), Hipparcos (Perryman et al. 1997), 2MASS (Skrutskie et al. 2006), and the General Catalogue of Photometric Data (GCPD; Mermilliod et al. 1997). The GCPD is a compilation of published ground based observations that includes, among many others, $U B V$ Johnson, RI Cousins and Kron, and Stromgren $u v b y$. The GCPD data are of nonuniform quality compared to these other surveys.

Ground-based infrared photometry from the ISO preparatory observations in both the ESO and Tenerife photometric systems ${ }^{19}$ were also included. Finally, the FEPS team obtained BRVI photometry for several stars that did not have high-quality photometry available in the literature. The observations, data reduction, and measured photometry for these sources are presented in Appendix A.

\section{C2. SYNTHETIC PHOTOMETRY}

For wavelengths longer than $10 \mu \mathrm{m}$, the original Kurucz synthetic spectra are sampled at $10.02 \mu \mathrm{m}$, and then between 20 and $160 \mu \mathrm{m}$ in steps of $20 \mu \mathrm{m}$. For wavelengths longer than $10 \mu \mathrm{m}$, we resampled the Kurucz spectra at finer wavelengths by interpolating between model data points assuming a $S_{\nu} \propto \nu^{2}$ spectrum.

Synthetic fluxes were computed by multiplying a Kurucz synthetic spectrum with the spectral response of a photometric system. The spectral response, $T(\lambda)$, includes the detector quantum efficiency, the atmospheric transmission (if appropriate), the filter transmission, and any other optics whose characterizations are available (see Cohen et al. 1999 for details). The product of these three transmission functions are referred to as a FAD (i.e., filter+atmosphere+detector).

By definition, the bandwidth of the filter in wavelength and frequency units is

$$
\begin{aligned}
& \Delta \lambda=\int T(\lambda) / T_{\max } d \lambda, \\
& \Delta \nu=\int T(\lambda) / T_{\max } d \nu,
\end{aligned}
$$

where $T_{\max }$ is the peak transmission. Uncertainties in the bandwidths were computed by assuming a $5 \%$ uncertainty in the transmission at any given wavelength. The spectral irradiance, $I$, can be computed by integrating the spectrum, $S(\lambda)$, over the FAD as

$$
I=\int S(\lambda) T(\lambda) d \lambda .
$$

The corresponding (isophotal) flux density is then defined as

$$
\begin{aligned}
& S_{\lambda}=I / \Delta \lambda, \\
& S_{\nu}=I / \Delta \nu .
\end{aligned}
$$

Since observed optical and near-infrared flux densities are typically quoted in magnitudes, the synthetic measurements were converted to magnitudes based on the flux for a zero-magnitude star as

$$
m=-2.5 \log \left(\frac{S_{\lambda}}{\mathrm{ZP}}\right)+\mathrm{zpo}
$$

where ZP is the zero point of the photometric system, and zpo is the offset needed to convert the synthetic photometry to the observed photometric system. Martin Cohen and collaborators have produced a series of papers in which they define the zero points and zero point offsets for several photometric systems. We adopt the calibration by Cohen et al. (2003a) for 2MASS, Cohen et al. (2003b) for Tycho-2, Hipparcos, and Landolt BVRI, and Cohen et al. (1999) for ESO HK and Tenerife $H K$. For Stromgren photometry, we adopt the calibration of Gray (1998) but replace his flux density for Vega at $5556 \AA$ with that of Cohen et al. (1992) for consistency.

\section{C3. FITTING PROCEDURE}

The $\chi^{2}$ merit equation to determine the best fit Kurucz model is

$$
\chi^{2}=\sum_{i=1}^{N}\left\{\frac{\left[F_{i, \mathrm{obs}}-F_{i, \text { model }}\left(T_{\mathrm{eff}}, \mathrm{A}_{\mathrm{V}},[\mathrm{Fe} / \mathrm{H}], \log g, \Omega\right)\right]^{2}}{\left(\Delta F_{i, \mathrm{obs}}^{2}+\Delta F_{i, \text { model }}^{2}\right)}\right\}+\left(\frac{T_{\mathrm{eff}}-T_{\mathrm{eff}, \mathrm{o}}}{\Delta T_{\mathrm{eff}, \mathrm{o}}}\right)^{2},
$$

where $F_{i, \text { obs }}$ is the observed flux density typically expressed in magnitudes, $F_{i, \text { model }}$ is the model flux density that depends on the stellar effective temperature $\left(T_{\text {eff }}\right)$, visual extinction $\left(A_{V}\right)$, metallicity $([\mathrm{Fe} / \mathrm{H}])$, surface gravity $(\log g)$, and solid angle $(\Omega)$, and $T_{\text {eff,o }}$ is the nominal temperature of the star derived from the spectral type (if available).

19 See http://www.iso.vilspa.esa.es/users/expl_lib/ISO/wwwcal/isoprep/gbpp/photom. 
Equation (C7) was minimized using a modified version of the Levenberg-Marquardt method as implemented by the LMDIF routine in the MINPACK library. ${ }^{20}$ The model parameters are the solid angle of the star, the effective temperature, surface gravity, metallicity, and visual extinction. In practice, the metallicity and surface gravity was fixed to the values listed in Table 5 . The constraint in the fitting procedure is that the visual extinction is nonnegative. The initial values for $A_{V}$ and $T_{\text {eff }}$ were set based on the stellar properties (see Appendix B).

Fits were constrained using photometry at wavelengths between 0.4 and $2.5 \mu \mathrm{m}$ for most sources. A few sources have excesses at $K$-band (Silverstone et al. 2006) and the model was fitted to photometry between 0.4 and $1.2 \mu \mathrm{m}$. Shorter wavelength photometry, in particular $U$-band observations, were omitted since those data are difficult to calibrate from the ground and are sensitive to the stellar metallicity. Longer wavelengths were omitted to avoid having infrared excesses bias the model fits.

Uncertainties in the model flux densities were computed using a grid search around the best-fit model parameters. The size of the grid was \pm 3 times the nominal parameter uncertainties computed from the covariance matrix computed from the least-squares fit. At each point in the model grid, we computed model flux densities, including the Spitzer IRAC and MIPS photometric bands, as well as the $\chi^{2}$ between that model and the observed flux densities for photometric bands between 0.4 and $2.5 \mu \mathrm{m}$. The relative probability that the model at a given grid point can reproduce the observations is $e^{-\chi^{2} / 2}$. The probabilities over all grid points then yields the probability distribution of model flux densities.

It is not feasible to present the full probability distribution for each Spitzer photometric band and each star. We instead characterized the probability distribution for a photometric band by the nominal flux density, $F_{\text {model }}$, and the $1 \sigma$ uncertainty $\Delta F_{\text {model }}$. The nominal flux density is given by the flux density computed from the best-fit model parameters. The $1 \sigma$ flux uncertainty is defined as the smallest range of model flux densities about $F_{\text {model }}$ that encompasses $68 \%$ of the total probability. Results from the Kurucz-model fitting have been used by Kim et al. (2005), Hines et al. (2006), and Hillenbrand et al. (2008).

${ }^{20}$ See http://www.netlib.org/minpack.

Ardila, D. R., et al. 2004, ApJ, 617, L147

Bouwman, J., et al. 2008, ApJ, 683, 479

Breger, M. 1986, ApJ, 309, 311

Carpenter, J. M., Wolf, S., Schreyer, K., Launhardt, R., \& Henning, T. 2005, AJ, 129, 1049

Carpenter, J. M., et al. 2008, ApJ, submitted (arXiv: 0810.1003)

Chen, B., Vergely, J. L., Valette, B., \& Carraro, G. 1998, A\&A, 336, 137

Cohen, M., Megeath, S. T., Hammersley, P. L., Martín-Luis, F., \& Stauffer, J. 2003a, AJ, 125, 2645

Cohen, M. Walker, R. G., Barlow, M. J., \& Deacon, J. R. 1992, AJ, 104, 1650

Cohen, M., Walker, R. G., Carter, B., Hammersley, P. L., Kidger, M., \& Noguchi, K. 1999, AJ, 117, 1864

Cohen, M., Wheaton, W. A., \& Megeath, S. T. 2003b, AJ, 126, 1090

Crawford, D., \& Barnes, J. 1974, AJ, 79, 687

D’Antona, F., \& Mazzitelli, I. 1997, Mem. Soc. Astron. Italiana, 68, 807

Engelbracht et al. 2007, PASP, 119, 994

Evans, N. J., II, et al. 2003, PASP, 115, 965

Fazio, G., Hora, J. L, Allen, L. E., et al. 2004, ApJS, 154, 10

Gordon, K. D., et al. 2007, PASP, 119, 1019

Gray, D. F. 1992, The Observation and Analysis of Stellar Photospheres (Cambridge: Cambridge Univ. Press)

Gray, R. O. 1998, AJ, 116, 482

Hampel, F. 1974, J. AM. Statist. Assoc., 69, 383

Hillenbrand, L. A., et al. 2008, ApJ, 677, 630

Hines, D. C., et al. 2006, ApJ, 638, 1070

Hollenbach, D., et al. 2005, ApJ, 631, 1180

Høg, E., et al. 2000, A\&A, 355, L27

Houck, J., et al. 2004, ApJS, 154, 18

Houdashelt, M. L., Bell, R. A., \& Sweigart, A. V. 2000, AJ, 119, 1448

Kenyon, S. H., I\& Hartmann, L. 1995, ApJS, 101, 117

Kim, J. S., et al. 2005, ApJ, 632, 659

Landolt, A. U. 1992, AJ, 104, 340

Makovoz, D., \& Marleau, F. R. 2005, PASP, 117, 1113

Mamajek, E. E., Meyer, M. R., \& Liebert, J. 2002, AJ, 124, 1670

. 2006, AJ, 131, 2360

\section{REFERENCES}

Mathis, J. S. 1990, ARA\&A, 28, 37

Mermilliod, J.-C., Mermilliod, M., \& Hauck, B. 1997, A\&AS, 124, 349

Meyer, M. R., et al. 2004, ApJS, 154, 422 2006, PASP, 118, 1690

Meyer, M. R., et al. 2008, ApJ, 673, L181

Moro-Martín, A., et al. 2007, ApJ, 658, 1312

Nagao, M., \& Matsuyama, T. 1979, Comput. Graphics Image Processing, 9, 394

Naylor, T. 1998, MNRAS, 296, 339

Pascucci, I., et al. 2006, ApJ, 651, 1177 2007, ApJ, 663, 383

Paulson, D. B., Sneden, C., \& Cochran, W. D. 2003, AJ, 125, 3185

Perryman, M. A. C., et al. 1997, A\&A, 323, L49

Pinsonneault, M. H., Stauffer, J., Soderblom, D. R., King, J. R., \& Hanson, R. B. 1998, ApJ, 504, 170

Prosser, C. F. 1992, AJ, 103, 488

Reach, W. T., et al. 2005, PASP, 117, 978

Rieke, G., et al. 2004, ApJS, 154, 25 2008, AJ, 135, 2245

Sandage, A. 1972, ApJ, 178, 1

Silverstone, M. D., et al. 2006, ApJ, 639, 1138

Skrutskie, M. F., et al. 2006, AJ, 131, 1163

Stauffer, J. R., et al. 2005, AJ, 130, 1834

Taylor, B. J. 1986, ApJS, 60, 577 2006, AJ, 132, 2453

Welsh, B. Y., Crifo, F., \& Lallement, R. 1998, A\&A, 333, 101

Werner, M., et al. 2004, ApJS, 154, 1

Whiteoak, J. B. 1961, MNRAS, 123, 245

Williams, J. P., Najita, J., Liu, M. C., Bottinelli, S., Carpenter, J. M., Hillenbrand, L. A., Meyer, M. R., \& Soderblom, D. R. 2004, ApJ, 604, 414

Wright, C. O., Egan, M. P., Kraemer, K. E., \& Price, S. D. 2003, AJ, 125, 349

Zacharias, N., Urban, S. E., Zacharias, M. I., Wycoff, G. L., Hall, D. M., Monet, D. G., \& Rafferty, T. J. 2004, AJ, 127, 3043 